\title{
Spiral Motion Enhanced Elite Whale Optimizer for Global Tasks
}

\author{
GuoChun Wang, ${ }^{1}$ Wenyong Gui, ${ }^{2}$ Guoxi Liang $\mathbb{D}^{3},{ }^{3}$ Xuehua Zhao, ${ }^{4}$ Mingjing Wang $\mathbb{D},{ }^{5}$ \\ Majdi Mafarja, ${ }^{6}$ Hamza Turabieh, ${ }^{7}$ Junyi Xin $\mathbb{D}^{8},{ }^{8}$ Huiling Chen $\mathbb{D}^{2},{ }^{2}$ and Xinsheng Ma $\mathbb{D}^{9}$ \\ ${ }^{1}$ College of Applied Technology, Changchun University of Technology, Changchun, Jilin 130012, China \\ ${ }^{2}$ College of Computer Science and Artificial Intelligence, Wenzhou University, Wenzhou, Zhejiang 325035, China \\ ${ }^{3}$ Department of Information Technology, Wenzhou Polytechnic, Wenzhou 325035, China \\ ${ }^{4}$ School of Digital Media, Shenzhen Institute of Information Technology, Shenzhen 518172, China \\ ${ }^{5}$ Institute of Research and Development, Duy Tan University, Da Nang 550000, Vietnam \\ ${ }^{6}$ Department of Computer Science, Birzeit University, Birzeit, State of Palestine \\ ${ }^{7}$ Department of Information Technology, College of Computers and Information Technology, P.O. Box 11099, Taif University, \\ Taif 21944, Saudi Arabia \\ ${ }^{8}$ School of Information Engineering, Hangzhou Medical College, Hangzhou 311300, Zhejiang, China \\ ${ }^{9}$ Department of Mathematics, Zhejiang International Studies University, Hangzhou 310023, China
}

Correspondence should be addressed to Junyi Xin; xinjunyi@hmc.edu.cn, Huiling Chen; chenhuiling.jlu@gmail.com, and Xinsheng Ma; xsma@zisu.edu.cn

Received 3 May 2021; Revised 4 July 2021; Accepted 14 August 2021; Published 31 August 2021

Academic Editor: Yanxia Sun

Copyright (c) 2021 GuoChun Wang et al. This is an open access article distributed under the Creative Commons Attribution License, which permits unrestricted use, distribution, and reproduction in any medium, provided the original work is properly cited.

\begin{abstract}
The whale optimization algorithm (WOA) is a high-performance metaheuristic algorithm that can effectively solve many practical problems and broad application prospects. However, the original algorithm has a significant improvement in space in solving speed and precision. It is easy to fall into local optimization when facing complex or high-dimensional problems. To solve these shortcomings, an elite strategy and spiral motion from moth flame optimization are utilized to enhance the original algorithm's efficiency, called MEWOA. Using these two methods to build a more superior population, MEWOA further balances the exploration and exploitation phases and makes it easier for the algorithm to get rid of the local optimum. To show the proposed method's performance, MEWOA is contrasted to other superior algorithms on a series of comprehensive benchmark functions and applied to practical engineering problems. The experimental data reveal that the MEWOA is better than the contrast algorithms in convergence speed and solution quality. Hence, it can be concluded that MEWOA has great potential in global optimization.
\end{abstract}

\section{Introduction}

Optimization tasks can be classified into many forms according to the relation and number of objectives or hybrid methods, such as robust optimization [1], large scale optimization [2,3], multiobjective [4], fuzzy optimization [5], and many objectives $[6,7]$. One of the classes of the optimizers is based on an iteration-based evolution of the swarm. At present, metaheuristic methods have attracted more and more attention from the public [8-22]. Because optimization problems appear in many fields of science, and they can be a solution to many fields like support vector machines [20, 23], feature selection [17, 24-28], extreme learning machine (ELM) [29-34], bankruptcy prediction $[19,35-38]$, engineering design $[16,18,39-43]$, optimal resource allocation [44], monitoring [45-48], parameters optimization [49], temperature optimization [50], intelligent damage detection [51, 52], smart grid [53], image enhancement optimization [54], image and video handling [55-60], medical image recognition [61], and image segmentation [62, 63], its randomness, diversification, and strengthening ability can effectively solve high nonlinearity 
and multilocal optimization problems. It can maximize the relationship between resources and interests and create maximum benefits under the condition of limited resources.

Swarm methods have emerged in many fields as they can deal with a wide variety of problems in a flexible way. Some well-distributed techniques are slime mould algorithm [64], Harris hawks optimization (HHO) [65], naked mole-rat algorithm (NMR) [66], hunger games search (HGS) [67], moth search algorithm (MSA) [68], monarch butterfly optimization (MBO) [69], krill herd algorithm (KH) [70], teaching-learning-based optimizer (TLBO) [71], differential evolution (DE) [72], and differential search (DS) [73]. The exploratory and exploitative abilities of the swarm-based method can be a good evolutionary basis to be applied to areas such as computer vision [74], deployment optimization [75], enhancement of the transportation networks [76], optimization of the tasks of deep learning [77-79], improvement of the prediction methods $[80,81]$, and decisionmaking technique [82-84]. One of the possible methods is the whale optimization algorithm (WOA), which is an intelligent optimization algorithm presented by Mirjalili and Lewis [85] in 2016. The algorithm mainly focuses on the whale's hunting behavior and the prey's predatory behavior to seek the optimal solution to the problem. Although WOA has the advantages of fewer parameters and strong global convergence, the standard WOA still possesses the problems of slow convergence speed and low convergence accuracy. Thus, many improved versions can be found in the literature. Wang et al. [86] devised the MOWOA, an opposition-based multiobjective WOA using global grid ranking, using multiple parts to improve optimization performance. In order to solve the problems of poor exploration and local optimal stagnation of WOA, Salgotra et al. [87] proposed an improved WOA based on mechanisms such as oppositionbased learning, exponentially decreasing parameters, and elimination or reinitialization of the worst particles. The improved algorithm has been experimentally demonstrated to have a large improvement in performance. Sun et al. [88] enhanced the WOA with the strategy of quadratic interpolation (QIWOA). The algorithm introduced new parameters to effectively search for solution spaced and handled premature convergence and adopted the quadratic interpolation around the best individual to enhance its exploitation capability and solution precision. Agrawal et al. [89] combined quantum concepts with the WOA, adopting the quantum bit representation of population agents and the quantum rotation gate operator as mutation operators to improve the exploration and exploitation ability of classical WOA. Hussein et al. [90] handled binary optimization problems by using the basic version of WOA and devising two transfer functions (S-shaped and V-shaped) to map the continuous search space into binary ones. Luo et al. [91] integrated three strategies with the original approach to obtain a better balance between exploration and exploitation trends. Firstly, the chaos initialization phase is utilized to start a group of chaos-triggered whales at the initial phase. Then, the diversity level of the evolutionary population is enhanced by the Gaussian mutation. Finally, the chaotic local search, combined with the "narrowing" strategy, is utilized to raise the original optimizer's utilization tendency. The effect of the signal is due to the base concepts in chaos theory [59, 92-94]. Sun et al. [95] proposed a nonlinear dynamic control parameter updating strategy based on cosine function and integrated this strategy and Lévy flight strategy into WOA (MWOA). Hemasian-Etefagh and Safi-Esfahani et al. [96] introduced a new idea (called GWOA) in whale grouping to overcome the early convergence problem. Elaziz and Mirjalili [97] integrated chaotic mapping and opposition-based learning into the algorithm and used the differential evolution (DE) algorithm to automatically select the chaotic graph and part of the population to alleviate the defects (DEWCO). Guo [98] devised an enhanced WOA by using the strategy of social learning and wavelet mutation. A new linear incremental probability is designed to increase the capability of global search. According to the principle of social learning, an individual's social network is constructed by using social hierarchy and social influence. So as to learn the exchange and sharing of information among groups, they established an adaptive neighborhood learning strategy on the basis of the network relationship. The Morlet wavelet mutation mechanism is adopted to learn the dynamic adjustment of mutation space, thus enhancing the capability of the algorithm to get rid of the local optimum.

WOA and its improved versions are often used to solve some practical application problems. Revathi et al. [99] devised an optimization scheme with the brainstorm WOA (BS-WOA) to identify the key used to improve the data structure's privacy and practicability by amending the database. Gong et al. [100] used an improved edition of the WOA to determine the optimal features and amend the classification's artificial neural network weights. The model is simulated on FLAIR, T1, and T2 data sets, showing that the presented model has a robust diagnostic capability. The model was then used to diagnose common diseases such as breast cancer, diabetes, and erythema squamous epithelium. Zhang et al. [101] utilized the best convolutional neural network (CNN) to process the skin disease image and adopted the improved WOA to optimize the CNN. Xiong et al. [102] devised an enhanced WOA, called IWOA, to accurately optimize different PV models' parameters, a typical complex nonlinear multivariable strong coupling optimization problem. Petrović et al. [103] analyzed the scheduling problem of a single mobile robot, and the best transportation method of raw materials, goods, and parts in an intelligent manufacturing system was found through WOA. Li et al. [104] used WOA to modify the input weight and hidden layer bias of extreme learning machine (ELM) and used this model to assess the aging of insulated gate bipolar transistor module. Akyol and Alatas [105] adopted WOA for emotional analysis, which is a multiobjective problem. Qiao [106] introduced adaptive search and encircling mechanism, spiral position, and jump behavior to enhance the efficiency of WOA and used the improved algorithm to predict short-term gas consumption. Lévy flight and pattern search were embedded into WOA for parameter estimation of solar cell and photovoltaic system [107]. 
Although WOA has significantly improved its performance and robustness compared with other metaheuristics algorithms, it is still not free from the dilemma of easily falling into local optimal solutions and other problems, and the same phenomenon of low solution accuracy and slow convergence exists when solving function problems. So, this paper proposes an improved variant of WOA, which is named MEWOA. We introduce two strategies: elite strategy and spiral motion from moth flame optimization (MFO) $[12,108,109]$, which significantly strengthens the convergence accuracy and speed of the basic WOA easier to jump out of local optimum. To further verify the performance of MEWOA, the algorithm is also utilized to solve practical engineering problems. The results reveal that MEWOA is superior to the other algorithms with high solution quality and convergence speed.

The main contributions of this study can be summarized as follows:

(i) Aiming at overcoming the problems of WOA, we introduce elite strategies as well as spiral motion into WOA to improve the diversity of populations while enhancing optimal solution selection and finally propose an improved WOA (MEWOA)

(ii) The MEWOA is compared with some with metaheuristic algorithms and advanced algorithms on function test sets such as CEC2017 and CEC2014, respectively, and satisfactory results are obtained

(iv) The proposed MEWOA has achieved excellent results in three typical engineering problems

This paper is structured as follows. Section 2 briefly introduces WOA, elite strategy, and MFO. Section 3 describes MEWOA. In Section 4, a range of experiments is conducted based on MEWOA to demonstrate the proposed algorithm's performance. In Section 5, the full content is summarized, and the future research direction is pointed out.

\section{Background Knowledge}

2.1. Whale Optimization Algorithm (WOA). WOA is a new metaheuristic algorithm devised by Mirjalili and Lewis [85] based on the Bubble-net behavior of humpback whales during hunting. In this algorithm, each humpback's position represents a feasible solution. Humpback whales hunt by producing unique bubbles along with a circular or "9" shaped path. According to such a phenomenon, the authors' mathematical models include the following three steps: random search, encircling prey, and attacking prey.

2.1.1. Random Search. Each agent's position is randomly generated to find prey. Moreover, the specific process is as follows:

$$
\begin{aligned}
\vec{D} & =\left|\vec{C} \vec{X}_{\text {rand }}^{d}-\vec{X}^{d}(t)\right|, \\
\vec{X}^{d}(t+1) & =\vec{X}_{\text {rand }}^{d}-\vec{A} \cdot \vec{D},
\end{aligned}
$$

where $\vec{X}_{\text {rand }}^{d}$ is the position of the $d$-th dimension in the randomly selected whale, $\vec{X}^{d}$ denotes the position of the current individual in $d$-th dimension, $t$ means the current number of iterations, the calculation result of $\vec{D}$ denotes the distance between the random individual and the current individual, and $\vec{A}$ and $\vec{C}$ are the coefficients as shown in the following formulas.

$$
\begin{aligned}
& \vec{A}=2 \vec{a} \cdot \vec{r}_{1}-\vec{a}, \\
& \vec{C}=2 \cdot \vec{r}_{2},
\end{aligned}
$$

where $\vec{a}$ is a constant that will linearly lessen from 2 to 0 , and $\vec{r}_{1}$ and $\vec{r}_{2}$ are random numbers in $[0,1]$.

2.1.2. Encircling Prey. When searching for the prey, the mathematical model is as follows:

$$
\begin{aligned}
\vec{D} & =\left|\vec{C} \vec{X}_{\text {best }}^{d}(t)-\vec{X}^{d}(t)\right|, \\
\vec{X}^{d}(t+1) & =\vec{X}_{\text {best }}^{d}(t)-\vec{A} \cdot \vec{D},
\end{aligned}
$$

where $\vec{X}_{\text {best }}^{d}(t)$ reveals the position of the $d$-th dimension in the best individual so far, $\vec{X}^{d}$ denotes the position of the current individual in $d$-th dimension, and the calculation result of $D$ denotes the distance between the best individual and the current individual.

2.1.3. Attacking Prey. On the basis of the hunting behavior of humpback whale, it swims in a spiral motion, so the mathematical model of hunting behavior is devised as follows:

$$
\vec{X}^{d}(t+1)=\vec{X}_{\text {best }}^{d}(t)+\vec{D}_{p} \cdot e^{b l} \cdot \cos (2 \pi l),
$$

where $\vec{D}_{p}=\left|\vec{X}_{\text {best }}^{d}(t)-\vec{X}^{d}(t)\right|$ denotes the distance between the whale and its prey, $b$ is a constant utilized to define the shape of the spiral, and $l$ is a random number in $[-1,1]$.

As the whale approaches its food in a spiral shape, it also shrinks its encircling circle. Therefore, $P_{i}$ is adopted to realize this synchronous behavior model, and Mirjalili sets $P_{i}$ as 0.5 to change the position of the whale between the constricted encircling mechanism or the spiral model. The concrete model is shown as follows:

$$
\vec{X}^{d}(t+1)= \begin{cases}\vec{X}_{*}^{d}(t)-\vec{A} \cdot \vec{D}, & p<P_{i}, \\ \vec{X}_{\text {best }}^{d}(t)+\vec{D}_{p} \cdot e^{b l} \cdot \cos (2 \pi l), & p \geq P_{i},\end{cases}
$$

where $p$ is a random number in $[-1,1]$. When $|\vec{A}|<1$ and $p<P_{i}$, the current position of $\vec{X}_{*}^{d}(t)$ means the $\vec{X}_{\text {best }}^{d}(t)$, and the whale updates the formula of encircling its prey. 
Otherwise, the agent updates the position by the randomly selected reference whale.

2.2. Elite Strategy. According to the position of the original population $\vec{X}$, we introduce a new population $\vec{X}_{1}$, according to the value of fitness Fitness $s_{1}$. Then, $\vec{X}$ and $\vec{X}_{1}$ are combined to form the population $\vec{X}_{2}$ sorted by fitness Fitness $s_{2}$, and the top $N$ is selected. The pseudocode of elite strategy is shown in Algorithm 1.

We know that the population obtained by random initialization can satisfy the search in the global solution, but such a search is not targeted. If the invalidity of some spatial regions has been proved during the first initialization, it is still possible to search for these useless regions when the random search is performed again, which leads to a waste of resources. The addition of the elite strategy can solve this problem better. While satisfying the global search, the population search will not search the invalid solution space again after the population search again but search again in the space where the optimal solution may exist, which can improve the efficiency of the algorithm to a greater extent. Through the elite strategy, new populations are generated by ranking the original populations according to their fitness values, after which the two are combined and from which the optimal top $N$ populations of individuals are then selected. Doing so is the selection of the optimal individuals each time and ultimately improves the overall population quality.

2.3. Moth Flame Optimization (MFO). MFO is a new swarm intelligence optimization algorithm proposed by $\mathrm{Xu}$ et al. $[12,108,109]$. It is inspired by the unique flight mode of moth named transverse orientation for navigation at night. In this algorithm, the set of moths $M$ can be illustrated as

$$
\vec{M}=\left[\begin{array}{ccc}
\vec{M}_{11} & \cdots & \vec{M}_{1 j} \\
\vdots & \cdots & \vdots \\
\vec{M}_{i 1} & \cdots & \vec{M}_{i j}
\end{array}\right]
$$

where $\vec{M}_{i j}$ is the $j$-th position corresponding to the $i$-th moth. Assuming the flame set is $\vec{F}, \vec{F}{ }_{i j}$ is the $j$-th position corresponding to the $i$-th flame, and the flame set can be expressed as follows:

$$
\vec{F}=\left[\begin{array}{ccc}
\vec{F}_{11} & \cdots & \vec{F}_{1 j} \\
\vdots & \cdots & \vdots \\
\vec{F}_{i 1} & \cdots & \vec{F}_{i j}
\end{array}\right] .
$$

Each agent updates its position according to the following expression:

$$
\vec{M}_{i}=\vec{S}\left(\vec{M}_{i}, \vec{F}_{i}\right)
$$

where $\vec{M}_{i}$ is the $i$-th moth, $\vec{F}_{i}$ is the $i$-th flame, and $\vec{S}$ is the helix function.

$$
\vec{S}\left(M_{i}, F_{j}\right)=\vec{D}_{i} \cdot e^{b t} \cdot \cos (2 \pi t)+\vec{F}_{j},
$$

where $\vec{D}_{i}$ denotes the linear distance between the $i$-th moth and the $j$-th flame; $b$ means the defined helix shape constant; $t$ denotes a random number in the interval $[-1,1]$.

To help the moth escape from the local optimum, the number of flames will decrease during the iteration:

$$
\text { flame.no }=\operatorname{round}\left[N-l \times \frac{N-1}{T}\right],
$$

where $l$ denotes the number of the current iteration; $N$ means the maximum quantity of flames; $T$ means the maximum quantity of iterations.

The process of MFO is summarized as follows:

(1) Initialize the population and calculate the fitness values of the population

(2) The fitness values are sorted; calculate the location of the flame and its fitness value

(3) Calculate the number of flames according to equation (12)

(4) Calculate the linear distance between the moth and the corresponding flame and substitute it into equation (11) to obtain the updated value

(5) Calculate the fitness value according to the updated moth population

(6) Judge whether the termination condition is met; otherwise, jump to Step 2

The strategies in the MFO give good access to the most optimal population individuals, i.e., the corresponding flame positions. Because the flame position is obtained with respect to the moth population, the flame position is obtained after the fitness value is calculated and ranked for the moth individuals, and with iteration, the flame position is selected only for the better individuals in the moth population. Therefore, applying MFO to WOA can effectively enhance the local search capability of the algorithm.

\section{Proposed Method}

In this section, the MEWOA is illustrated in detail. The flowchart of the proposed MEWOA is presented in Figure 1. MEWOA incorporates the elite strategy and MFO algorithm for balancing the capability of exploration and exploitation. The algorithm first uses an elite strategy to generate a highquality candidate population. Based on this population, the MFO algorithm is used to form a better population, which can help the algorithm possess the fast convergence, find out the optimal solution, and effectively avoid premature stagnation. The pseudocode of MEWOA is illustrated in Algorithm 2.

The computational complexity of MEWOA is due to the population size $(N)$, dimension size (Dim), and maximum evaluation iterations (Max_FEs). Iteration number $(l)$ is related to the maximum evaluation number and population size, $\quad l=M a x \_F E s / N$. Time complexity expression: $O($ MEWOA $)=O($ evaluation of the fitness $)+t \times(O($ elite 
Calculate the fitness of the original population Fitness ${ }_{1}$;

Sort Fitness $s_{1}$ and rearrange $\vec{X}$ by serial number to get $\vec{X}_{1}$; Combine $\vec{X}$ and $\vec{X}_{1}$ to form $\vec{X}_{2}$;

Calculate the fitness of the population $\vec{X}_{2}$ recorded as Fitness ${ }_{2}$; Sort Fitness 2 and select the top N;

Algorithm 1: Pseudocode of elite strategy.

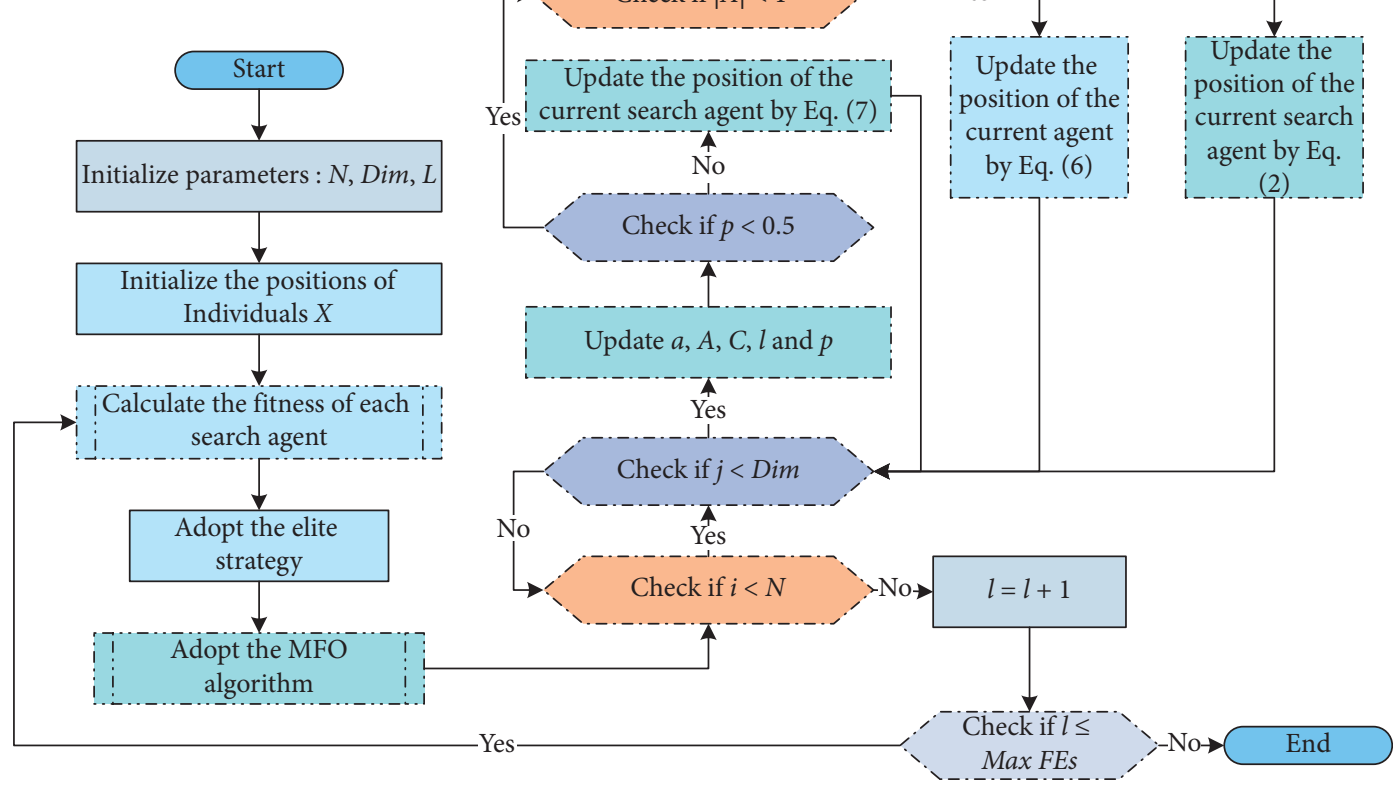

FIgURE 1: Flowchart of MEWOA.

Initialize the population $\vec{X}_{i}(i=1,2, \ldots, N), N, l$, dim, Max_FEs

Calculate the fitness of each search agent

$\vec{X}^{*}=$ the best search agent

while $(l \leq$ Max_FEs $)$

Adopt the Elite strategy

Adopt the MFO algorithm

for each whale

Update $\vec{a}, \vec{A}, \vec{C}, l$ and $p$

if $(p<0.5)$

if $(|\vec{A}|<1)$

Update the position of the current agent by the equation (5) elseif $(|\vec{A}| \geq 1)$

Select a random search agent $\left(\vec{X}_{\text {rand }}\right)$

Update the position of the current search agent by equation (2) end if

elseif $(p \geq 0.5)$

Update the position of the current search agent by equation (6)

end for end if

Check if any search agent goes beyond the search space and amend it

Calculate the fitness of each search agent

Update $\vec{X}^{*}$ if there is a better solution $l=l+1$

end while

return $\vec{X}^{*}$

Algorithm 2: Pseudocode of MEWOA algorithm. 
strategy $)+O(\mathrm{MFO})+O$ (estimation of the fitness $)+O(\mathrm{WOA}))$. The complexity of the estimation of the fitness is $O(N)$, the complexity of elite strategy is $O(0)$, the complexity of MFO is $O(N \times D i m)$, and the complexity of WOA is $O(N \times D i m)$. So, the whole time complexity is $O($ MEWOA $)=O(N)+O(2 \times N \times \operatorname{Dim}+N) \times t$. The time complexity of the original WOA is $O(W O A)=$ $t \times(O$ (evaluation of the fitness $)+O($ WOA $))$. So, the whole complexity of the original WOA is $O($ WOA $)=$ $t \times(O(N)+O(N \times D i m))$. The increased complexity compared to the two is $O(N)+O(N \times \operatorname{Dim}) \times t$.

\section{Experimental Studies}

In this section, we further verify the performance of MEWOA. Firstly, the combination of strategies and the stability of the algorithm is analyzed. Next, on the CEC 2017 competition data set, we adopt several advanced versions of WOA as a comparison. At last, the algorithm is applied to three practical engineering problems.

The related experiments are conducted under the Windows Server 2012 R2 operating system adopting MATLAB R2014a software, and the hardware platform is configured with Intel $(\mathrm{R})$ Xeon $(R)$ Sliver $4110 \mathrm{CPU}$ $(2.10 \mathrm{GHz})$ and $16 \mathrm{~GB}$ RAM.

4.1. Benchmark Functions and Performance Evaluation Measures. This experiment adopts the IEEE CEC 2017 competition data set as a test function, which can effectively estimate the algorithm's ability. To ensure the experiment's fairness, the involved algorithms are evaluated under the same conditions: the overall scale and the maximal iteration numbers are set as 300000 and 150000, respectively. This is to ensure there is no bias and unfair setting that make the tests toward a specific method, as per artificial intelligence works [110-112]. The related algorithms are estimated 30 times on each benchmark function independently. Friedman's test [113] is a nonparametric statistical comparison test that can evaluate the experimental results. It is usually utilized to seek the difference between multiple test results and rank all algorithms' average performance to make a statistical comparison and get the ARV value (average ranking value). For the statistical test, paired Wilcoxon signed-rank test [114] is also adopted in this experiment. Wilcoxon signed-rank test can compare the performance between two algorithms. When the $p$ value is less than 0.05 , it indicates that the performance of MEWOA is statistically significantly improved compared to another algorithm.

4.2. Impacts of Components. MEWOA is a novel group intelligence algorithm introducing the two mechanisms of the MFO [108] algorithm and Elite Opposition-Based Learning (EOBL) [115] into the basic WOA. To better understand the influence of each mechanism on the performance of the WOA, we compare the model MWOA after the MFO algorithm, the EWOA model after the EOBL mechanism, and the MEWOA model after the two mechanisms which are integrated at the same time to study the
TABLE 1: Various WOAs with seven strategies.

\begin{tabular}{lcc}
\hline & $M$ & $O$ \\
\hline WOA & 0 & 0 \\
MWOA & 1 & 0 \\
EWOA & 0 & 1 \\
MEWOA & 1 & 1 \\
\hline
\end{tabular}

impact of each mechanism on the algorithm. In Table 1, "M" represents the MFO algorithm, and "E" represents the EOBL mechanism. Furthermore, " 1 " indicates that this mechanism is used in the WOA algorithm, and " 0 " indicates that the corresponding mechanism is not used. Table 2 reveals the test data of the four algorithms in the CEC2017 [116] functions. This experiment is carried out under the same condition. The dimension is set to 30 , the number of particles is set to 30 , and the maximum number of evaluations is set to 150,000 times. For obtaining the average results, each algorithm is run 30 times independently.

We test the impact of different mechanisms on the algorithm on 30 benchmark functions in CEC2017. Table 2 shows the comparison results of various models. We have listed the average results and standard deviations of different algorithms running on the test function 30 times, and the optimal values are shown in bold. On 30 test functions, the improved algorithm MEWOA has achieved the optimal solutions on most functions. MEWOA has significant advantages compared with MWOA and EWOA. The experimental results reveal that the $\mathrm{MFO}$ algorithm and $\mathrm{EOBL}$ mechanism added to the WOA algorithm can effectively enhance the performance of the original WOA and enhance the ability to search for optimal solutions.

To further study the improved MEWOA algorithm's performance, we performed the following analytical experiments on the CEC2017 functions. Figure 2 demonstrates the results of the feasibility analysis of MEWOA, where the original WOA algorithm is chosen for comparison. The graph in the first column (a) shows the three-dimensional location distribution of the MEWOA search history. The second column (b) graph reveals the two-dimensional location distribution of the MEWOA search history. The graph in the third column (c) shows the trajectory of MEWOA during the iterative process. The graph in the fourth column (d) shows the average fitness variation over the iterative process. The graph in the fifth column (e) demonstrates the convergence curve of the algorithm.

The black dots in Figure 2(b) show the algorithm's historical search positions, and the red dots show the optimal solutions' positions. It can be visualized from the figure that most of the black dots are clustered around the red dots, and a small portion of the black dots are scattered all over the solution space. The individual trajectories in Figure 2(c) show that the individuals fluctuate significantly in the first and middle stages and gradually stabilize in the later stages. Both data show that the algorithm can search the whole solution space as much as possible and then determine the region where the optimal solution is located for further exploitation. Figure 2(d) shows that the algorithm's average fitness curve maintains a constant decline throughout the 
TABLE 2: Comparison results of MEWOA and other variants.

\begin{tabular}{|c|c|c|c|c|c|c|}
\hline Fun & Item & MEWOA & EWOA & MWOA & $\mathrm{MFO}$ & WOA \\
\hline \multirow{2}{*}{ F1 } & AVG & $1.1402 E+05$ & $6.2606 E+09$ & $5.1740 E+07$ & $1.1003 E+10$ & $2.8434 E+07$ \\
\hline & STD & $3.3373 E+05$ & $3.1034 E+09$ & $6.9757 E+07$ & $8.7195 E+09$ & $2.4863 E+07$ \\
\hline \multirow{2}{*}{$\mathrm{F} 2$} & AVG & $1.0140 E+13$ & $2.8712 E+36$ & $8.7045 E+26$ & $4.5123 E+37$ & $8.1117 E+25$ \\
\hline & STD & $2.1249 E+13$ & $1.3645 E+37$ & $4.7319 E+27$ & $1.6948 E+38$ & $4.0528 E+26$ \\
\hline \multirow{2}{*}{ F3 } & AVG & $1.3903 E+04$ & $2.5163 E+05$ & $2.1779 E+05$ & $7.4876 E+04$ & $2.0430 E+05$ \\
\hline & STD & $6.9178 E+03$ & $9.5273 E+04$ & $6.2175 E+04$ & $6.8751 E+04$ & $7.3817 E+04$ \\
\hline \multirow{2}{*}{$\mathrm{F} 4$} & AVG & $5.3104 E+02$ & $1.4024 E+03$ & $5.8750 E+02$ & $1.5023 E+03$ & $5.6894 E+02$ \\
\hline & STD & $5.0035 E+01$ & $8.0323 E+02$ & $3.9813 E+01$ & $8.8614 E+02$ & $3.5987 E+01$ \\
\hline \multirow{2}{*}{ F5 } & $\mathrm{AVG}$ & $7.0209 E+02$ & $8.4901 E+02$ & $7.9324 E+02$ & $7.1606 E+02$ & $7.8909 E+02$ \\
\hline & STD & $5.8959 E+01$ & $6.3694 E+01$ & $5.7745 E+01$ & $6.4386 E+01$ & $6.8153 E+01$ \\
\hline \multirow{2}{*}{ F6 } & AVG & $6.4492 E+02$ & $6.7827 E+02$ & $6.7206 E+02$ & $6.3434 E+02$ & $6.7004 E+02$ \\
\hline & STD & $1.1187 E+01$ & $1.5775 E+01$ & $1.2419 E+01$ & $1.2633 E+01$ & $9.8938 E+00$ \\
\hline \multirow{2}{*}{ F7 } & AVG & $9.6106 E+02$ & $1.3466 E+03$ & $1.2272 E+03$ & $1.1855 E+03$ & $1.2408 E+03$ \\
\hline & STD & $7.5270 E+01$ & $7.6342 E+01$ & $6.9796 E+01$ & $2.1546 E+02$ & $7.6205 E+01$ \\
\hline \multirow{2}{*}{ F8 } & AVG & $9.9622 E+02$ & $1.0891 E+03$ & $1.0221 E+03$ & $1.0117 E+03$ & $1.0283 E+03$ \\
\hline & STD & $4.8523 E+01$ & $5.7098 E+01$ & $4.4966 E+01$ & $5.7584 E+01$ & $5.3755 E+01$ \\
\hline \multirow{2}{*}{ F9 } & AVG & $5.3323 E+03$ & $1.5001 E+04$ & $9.0558 E+03$ & $7.5359 E+03$ & $9.2026 E+03$ \\
\hline & STD & $2.2701 E+03$ & $6.2589 E+03$ & $3.4108 E+03$ & $1.6460 E+03$ & $4.3758 E+03$ \\
\hline \multirow{2}{*}{ F10 } & $\mathrm{AVG}$ & $5.3003 E+03$ & $7.9193 E+03$ & $6.3360 E+03$ & $5.7461 E+03$ & $6.5544 E+03$ \\
\hline & STD & $6.1006 E+02$ & $7.5830 E+02$ & $7.4958 E+02$ & $8.7887 E+02$ & $8.6284 E+02$ \\
\hline \multirow{2}{*}{ F11 } & $\mathrm{AVG}$ & $1.2985 E+03$ & $5.7913 E+03$ & $2.3611 E+03$ & $6.5666 E+03$ & $2.4914 E+03$ \\
\hline & STD & $5.9662 E+01$ & $2.8798 E+03$ & $1.2251 E+03$ & $5.7601 E+03$ & $1.3764 E+03$ \\
\hline \multirow{2}{*}{ F12 } & $\mathrm{AVG}$ & $2.8637 E+06$ & $1.8066 E+08$ & $1.0806 E+08$ & $4.1075 E+08$ & $6.9061 E+07$ \\
\hline & STD & $1.8477 E+06$ & $1.2913 E+08$ & $6.0636 E+07$ & $7.4554 E+08$ & $5.6292 E+07$ \\
\hline \multirow{2}{*}{ F13 } & $\mathrm{AVG}$ & $3.1678 E+04$ & $8.7338 E+07$ & $1.7061 E+05$ & $3.8575 E+07$ & $1.4739 E+05$ \\
\hline & STD & $2.2382 E+04$ & $2.9375 E+08$ & $1.1901 E+05$ & $1.9330 E+08$ & $7.3276 E+04$ \\
\hline \multirow{2}{*}{ F14 } & AVG & $6.1824 E+04$ & $1.9699 E+06$ & $1.3829 E+06$ & $2.1637 E+05$ & $1.1689 E+06$ \\
\hline & STD & $3.6973 E+04$ & $2.6824 E+06$ & $1.8840 E+06$ & $3.7891 E+05$ & $1.1808 E+06$ \\
\hline \multirow{2}{*}{ F15 } & AVG & $1.7080 E+04$ & $3.5083 E+06$ & $9.6559 E+04$ & $6.0508 E+04$ & $9.2638 E+04$ \\
\hline & STD & $1.2528 E+04$ & $5.9417 E+06$ & $7.1048 E+04$ & $5.7199 E+04$ & $8.3603 E+04$ \\
\hline & AVG & $2.7163 E+03$ & $4.0865 E+03$ & $3.6880 E+03$ & $3.2619 E+03$ & $3.6476 E+03$ \\
\hline F16 & STD & $3.2745 E+02$ & $7.3533 E+02$ & $4.0789 E+02$ & $4.4991 E+02$ & $4.8167 E+02$ \\
\hline & AVG & $2.2809 E+03$ & $2.9371 E+03$ & $2.5306 E+03$ & $2.5576 E+03$ & $2.5618 E+03$ \\
\hline F17 & STD & $1.9868 E+02$ & $4.9570 E+02$ & $2.3127 E+02$ & $2.9339 E+02$ & $3.1216 E+02$ \\
\hline & AVG & $8.2210 E+05$ & $1.2431 E+07$ & $2.9497 E+06$ & $4.0601 E+06$ & $2.9801 E+06$ \\
\hline F18 & STD & $8.9710 E+05$ & $1.2645 E+07$ & $4.0345 E+06$ & $7.8932 E+06$ & $2.5286 E+06$ \\
\hline & $\mathrm{AVG}$ & $2.2487 E+04$ & $1.5354 E+07$ & $5.5705 E+06$ & $1.8640 E+07$ & $5.6004 E+06$ \\
\hline F19 & STD & $2.0128 E+04$ & $5.8271 E+07$ & $4.0800 E+06$ & $4.0707 E+07$ & $4.8343 E+06$ \\
\hline & $\mathrm{AVG}$ & $2.7115 E+03$ & $3.0666 E+03$ & $2.8007 E+03$ & $2.6387 E+03$ & $2.7859 E+03$ \\
\hline F20 & STD & $2.2438 E+02$ & $2.3437 E+02$ & $2.1679 E+02$ & $2.1030 E+02$ & $1.4944 E+02$ \\
\hline & $\mathrm{AVG}$ & $2.4777 E+03$ & $2.6424 E+03$ & $2.5940 E+03$ & $2.4995 E+03$ & $2.5964 E+03$ \\
\hline F21 & STD & $4.7650 E+01$ & $8.0860 E+01$ & $6.7087 E+01$ & $5.0812 E+01$ & $6.4482 E+01$ \\
\hline & AVG & $6.4893 E+03$ & $9.1461 E+03$ & $6.1745 E+03$ & $6.6092 E+03$ & $7.2471 E+03$ \\
\hline F22 & STD & $1.6129 E+03$ & $8.4869 E+02$ & $2.2847 E+03$ & $1.2145 E+03$ & $1.6189 E+03$ \\
\hline & AVG & $2.8732 E+03$ & $3.1343 E+03$ & $3.0388 E+03$ & $2.8338 E+03$ & $3.0702 E+03$ \\
\hline F23 & STD & $6.2221 E+01$ & $8.9745 E+01$ & $1.2308 E+02$ & $5.2984 E+01$ & $1.0063 E+02$ \\
\hline & $\mathrm{AVG}$ & $3.0054 E+03$ & $3.2910 E+03$ & $3.1764 E+03$ & $2.9872 E+03$ & $3.1805 E+03$ \\
\hline F24 & STD & $5.8026 E+01$ & $9.0717 E+01$ & $8.1690 E+01$ & $3.0441 E+01$ & $8.1683 E+01$ \\
\hline & AVG & $2.9082 E+03$ & $3.2249 E+03$ & $2.9964 E+03$ & $3.3361 E+03$ & $2.9930 E+03$ \\
\hline F25 & STD & $2.0562 E+01$ & $1.6505 E+02$ & $3.8915 E+01$ & $5.1024 E+02$ & $4.4618 E+01$ \\
\hline & AVG & $5.8834 E+03$ & $8.6678 E+03$ & $7.9037 E+03$ & $5.8878 E+03$ & $7.7769 E+03$ \\
\hline F26 & STD & $5.5664 E+02$ & $8.9188 E+02$ & $1.2502 E+03$ & $5.5639 E+02$ & $7.8980 E+02$ \\
\hline & AVG & $3.4081 E+03$ & $3.8352 E+03$ & $3.4215 E+03$ & $3.2540 E+03$ & $3.3830 E+03$ \\
\hline F27 & STD & $1.1014 E+02$ & $3.2857 E+02$ & $1.0341 E+02$ & $2.9023 E+01$ & $9.0797 E+01$ \\
\hline & AVG & $8.8194 E+03$ & $7.5059 E+03$ & $3.3450 E+03$ & $4.4173 E+03$ & $3.3436 E+03$ \\
\hline F28 & STD & $1.0576 E+03$ & $1.5877 E+03$ & $3.7885 E+01$ & $1.0738 E+03$ & $3.3948 E+01$ \\
\hline & AVG & $4.0622 E+03$ & $5.1185 E+03$ & $5.0380 E+03$ & $3.9958 E+03$ & $5.0393 E+03$ \\
\hline F29 & STD & $2.5075 E+02$ & $4.3072 E+02$ & $4.9458 E+02$ & $2.2794 E+02$ & $5.9330 E+02$ \\
\hline & AVG & $3.7243 E+04$ & $8.7945 E+06$ & $2.2134 E+07$ & $1.0549 E+06$ & $1.6396 E+07$ \\
\hline F30 & STD & $2.6056 E+04$ & $7.8295 E+06$ & $1.9372 E+07$ & $1.9420 E+06$ & $1.9789 E+07$ \\
\hline
\end{tabular}



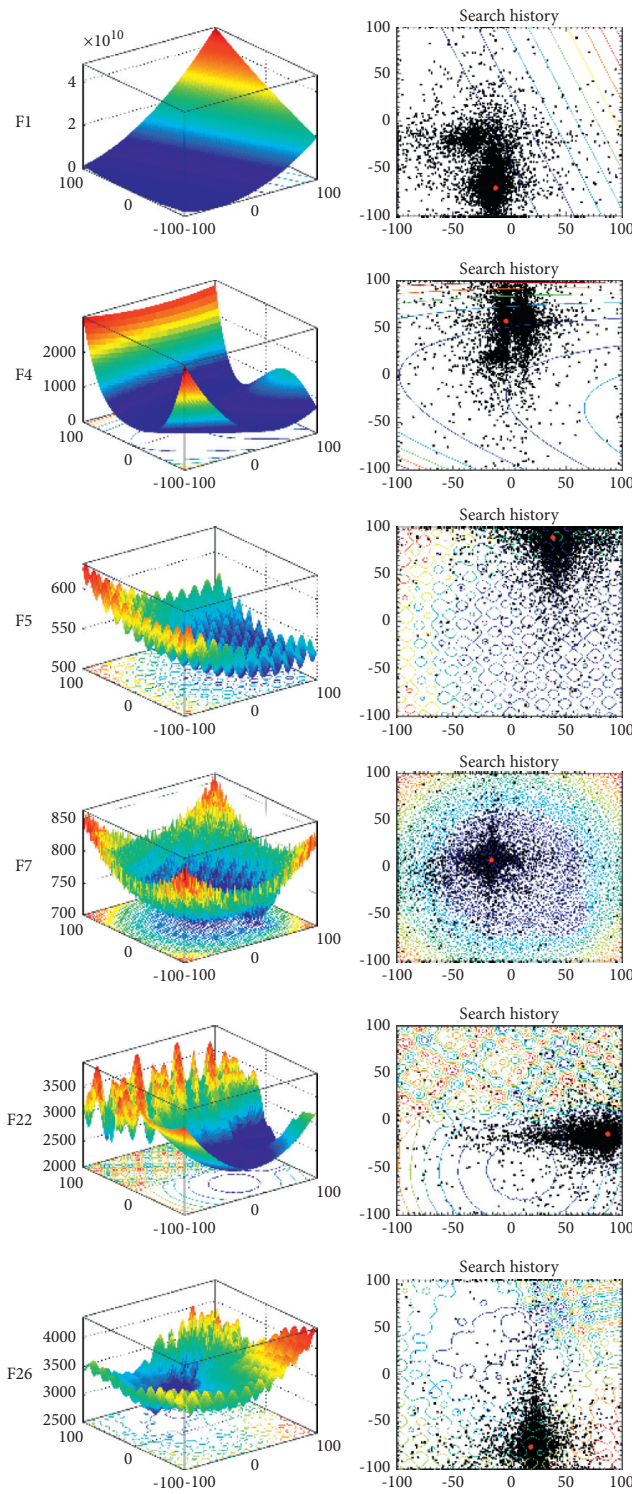

(a)

- MEWOA

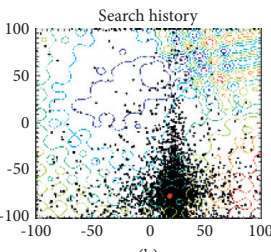

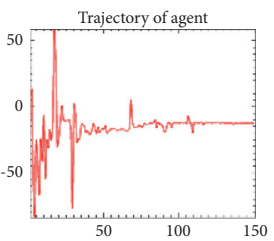
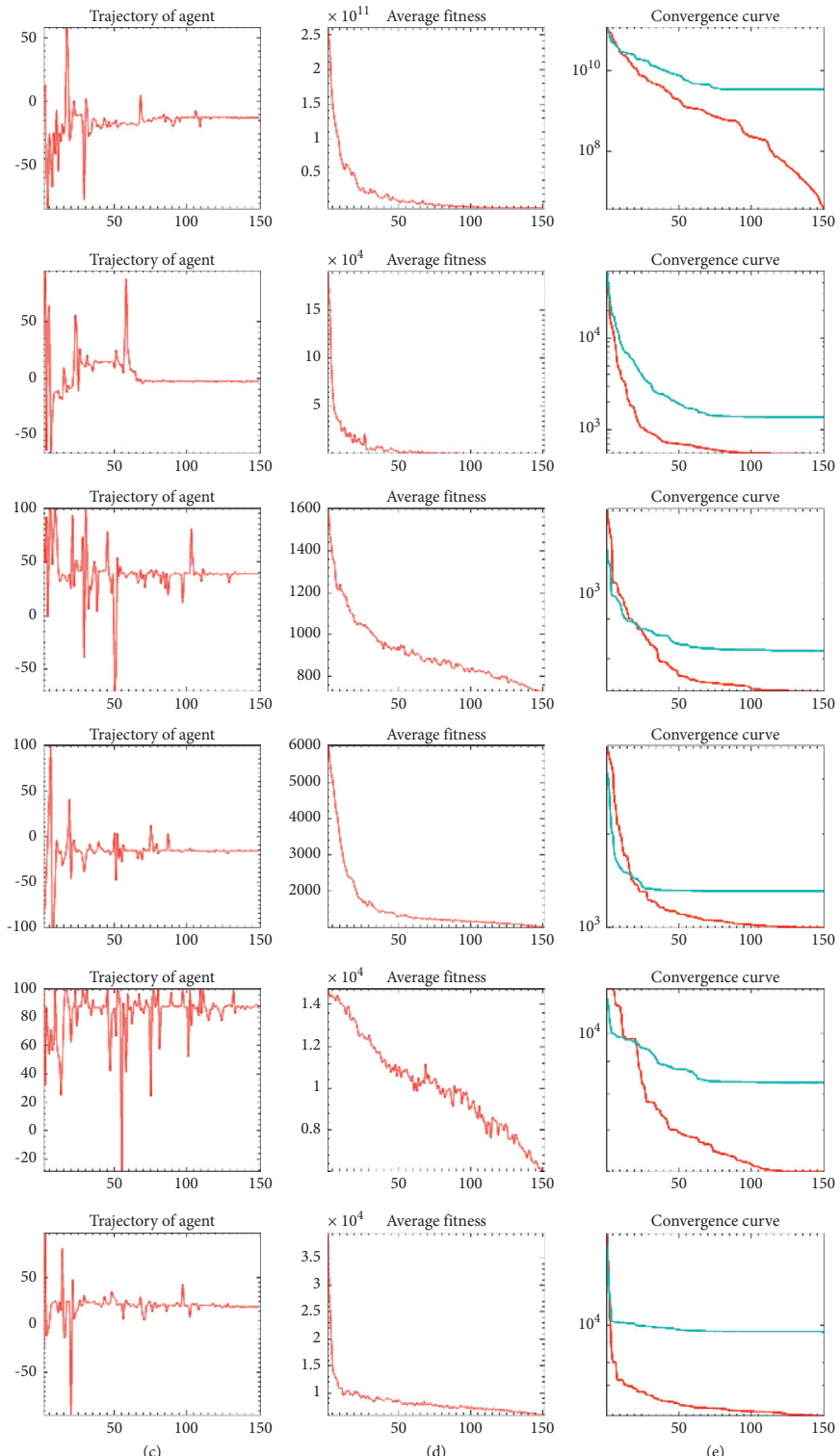

FIgURE 2: (a) Three-dimensional location distribution of MEWOA, (b) two-dimensional location distribution of MEWOA, (c) trajectory of MEWOA in the first dimension, (d) average fitness of MEWOA, and (e) convergence curves of algorithms.

iterations. F1, F4, F7, and F26 fall to lower fitness values early in the iteration. It shows that the algorithm exhibits good convergence ability on these functions. In Figure 2(e), it is more evident from the convergence curves of the two algorithms that MEWOA can find solutions with better quality.

This paper also analyzes the balance and diversity of these two algorithms on the CEC 2017 functions. Figure 3 demonstrates the results of the balanced analysis of MEWOA and WOA. The red and blue curves in the figure represent the exploration effect and exploitation effect, respectively. The higher the value of the curve, the more dominant the corresponding effect. A third curve is added to visualize the relationship between the two effects more clearly. When the value of the exploration effect is higher than or equal to the exploitation effect, the curve increases. Otherwise, the curve decreases. When the curve decreases to a negative value, it is set to zero.

The general algorithm always performs a global search first and then develops the target area locally after the target area is identified. Therefore, in the algorithm's balance analysis curve, the exploration curve always starts with a higher value, and MEWOA has no exception. From Figure 3, we can see that the exploration and exploitation curves of both algorithms fluctuate considerably. The exploitation effect occupies most of the time in both. On the selected functions, the exploration phase of MEWOA ends significantly earlier than that of WOA. The exploitation curves have been increasing since then, indicating that MEWOA spends more time exploiting the target area.

Figure 4 reveals the change of the algorithm diversity during the optimization process. From the figure, we can 
clearly see that the algorithm shows high population diversity at the beginning due to its random initialization. As the iterations progress, the algorithm keeps narrowing the search and reduces the population diversity. From the figure, we can see that the diversity curves of MEWOA and WOA are relatively similar. We know that both elite selection and MFO will make the algorithm converge faster in the early stage, and the population diversity will decline rapidly. However, the encircling mechanism, random search mechanism, and unique update method of WOA fluctuate between global and local during exploration. This success prevents MEWOA from converging too quickly in the early stage.

4.3. Scalability Test. To test the MEWOA algorithm's performance for searching the optimal solution in different dimensions, we conducted the test in two dimensions of 50 and 100 and compared it with six other algorithms. In the experiment, the number of particles is set to 30 . The maximum number of evaluations is set to 150,000 times. Each algorithm is independently run 30 times to take the average, and the CEC2017 test function is selected. The related results are demonstrated in Table 3, where AVG denotes the results' average, and STD means the standard deviation. Compared with other algorithms, the data shows that MEWOA has excellent advantages in processing single-mode functions in 50 and 100 dimensions. The improved MEWOA possesses more excellent performance than the other six improved WOA algorithms and also has a powerful ability to search for optimal solutions.

4.4. Comparison with Well-Established Methods. To investigate the improved MEWOA algorithm's performance and advantages in this paper, a comparative test is made with several improved WOA. These algorithms are very successful improved WOA with excellent search performance. In the test, the dimension of the particles is set to 30 , the number of particles is set to 30 , the maximum number of evaluations is set to 150,000 times, each algorithm is independently run 30 times to take the average, and the test function selects the CEC2017 test function. Table 4 lists the involved algorithms' comparison results using the average and standard deviation of each algorithm running 30 times on different test functions. The table reveals that the average and standard deviations obtained from the improved MEWOA in this paper are smaller than other comparison algorithms.

We use the Friedman [113] test to rank the algorithms' performance and the Friedman test to find the difference between the results of multiple tests, which are nonparametric statistical comparative tests. The Friedman test ranks the average scores of the involved algorithms and then conducts further statistical comparisons to obtain ARV (average ranking values) from the results. It can be realized from Table 4 that the enhanced algorithm in this paper possesses better performance than other comparison algorithms in test functions except for F22, F27, and F28. Wilcoxon's [114] rank-sum test is also utilized in this paper to test whether MEWOA is superior to the comparison algorithm. As the $p$ value is less than 0.05 , we can realize that the MEWOA is significantly better than the comparison algorithm in the current test function. As shown in Table 4, the $p$ value of MEWOA is less than 0.05 on most test functions, so the improved algorithm in this paper is better than other compared algorithms on most test functions.

Convergence speed and convergence accuracy are important indicators for investigating the performance of evolutionary algorithms. We have selected six representative test functions for learning the algorithm's effectiveness and search trends more quickly and clearly, which are shown in Figure 5, namely, F1, F10, F12, F18, F26, and F30. It can be seen that on test functions F1, F12, F18, and F30, the improved algorithm in this paper has not converged to the optimal value after 150,000 evaluations, and the convergence trend is much higher than other comparison algorithms. In all cases, the convergence accuracy of the MEWOA is greater than the peers.

4.5. Comparison with Representative Metaheuristic Algorithms. To better verify the performance of MEWOA, in this section, we will select some representative metaheuristics to compare with MEWOA. Among the algorithms involved in the comparison, there are classical algorithms, such as DE, as well as algorithms with good results proposed in the past years, such as MFO, and new algorithms proposed in recent years, such as SMA. The details are shown as follows.

\section{(i) $\mathrm{HHO}$}

(ii) SMA

(iii) Hunger games search (HGS) [67]

(iv) $\mathrm{DE}$

(v) $\mathrm{MFO}$

(vi) Cuckoo search (CS) [117]

(vii) Grasshopper optimization algorithm (GOA) [118]

The parameters of the experiments were set approximately the same as the previous experiments. The dimension of particles was set to 30 , the number of particles was set to 30 , and the maximum number of evaluations was set to 300,000 . The test function is IEEE CEC2017. Table 5 lists the experimental results of this experiment. In Table 5, AVG denotes the average value obtained for each algorithm after 30 independent tests on the corresponding function, STD denotes the corresponding standard deviation, and Rank denotes the ranking of the algorithm on each function. In addition, we used the Wilcoxon singed-rank test to calculate the $p$ value on the algorithms, the purpose of which is to obtain whether there is variability in the comparison results of the two algorithms. If the $p$ value is less than 0.05 , it means that the comparison between MEWOA and the corresponding algorithm is statistically significant, and vice versa, it means that the results do not have any significance.

There are 30 functions in the CEC2017 test set, which are divided into 4 categories, among which, F1-F3 are Unimodal functions, F4-F10 are Multimodal functions, F11-F20 are Hybrid functions, and F21-F30 are Composition functions. In Figure 6, we have selected two functions from each class and depicted the convergence curves of MEWOA with other metaheuristic algorithms. 

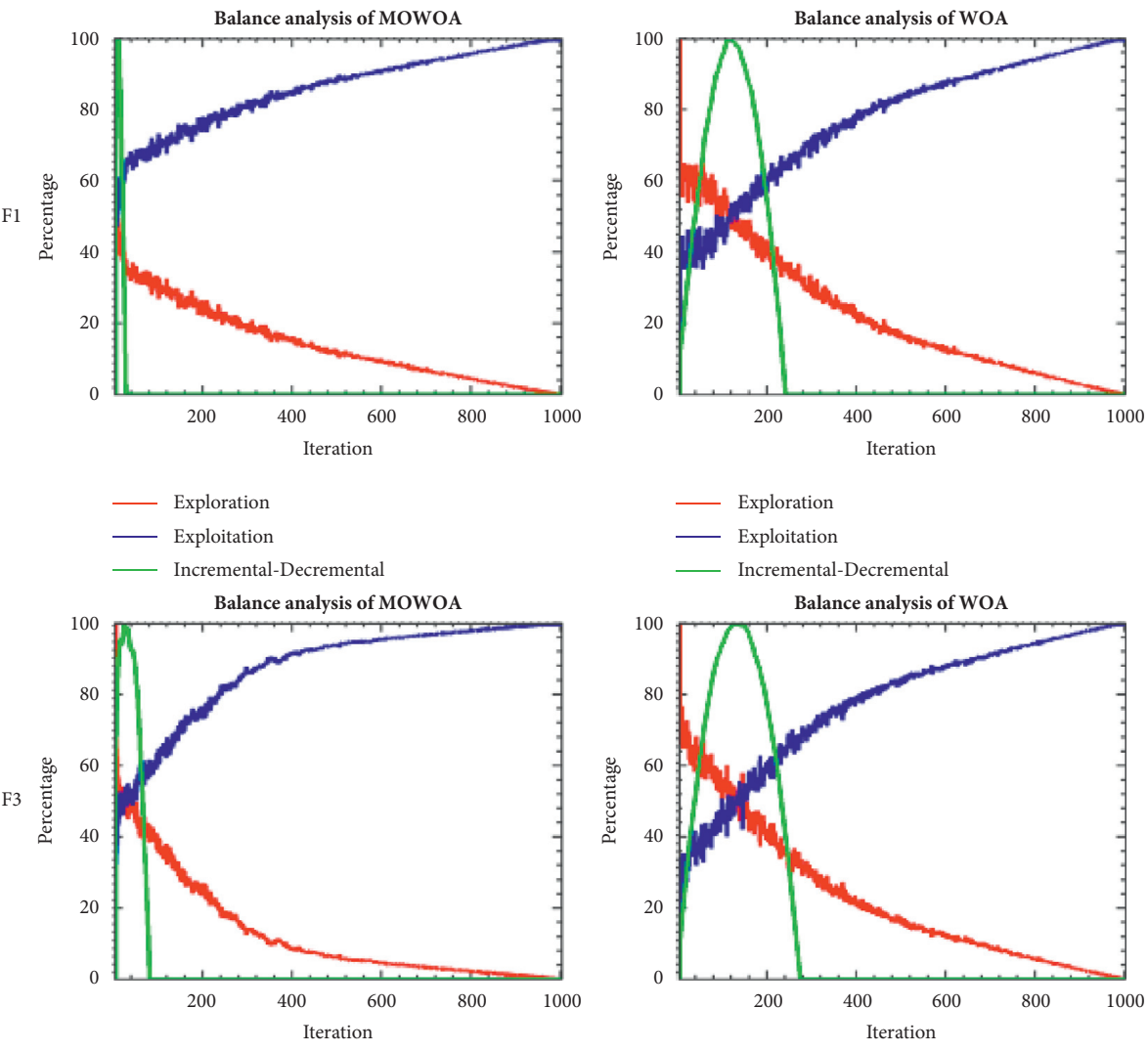

F3
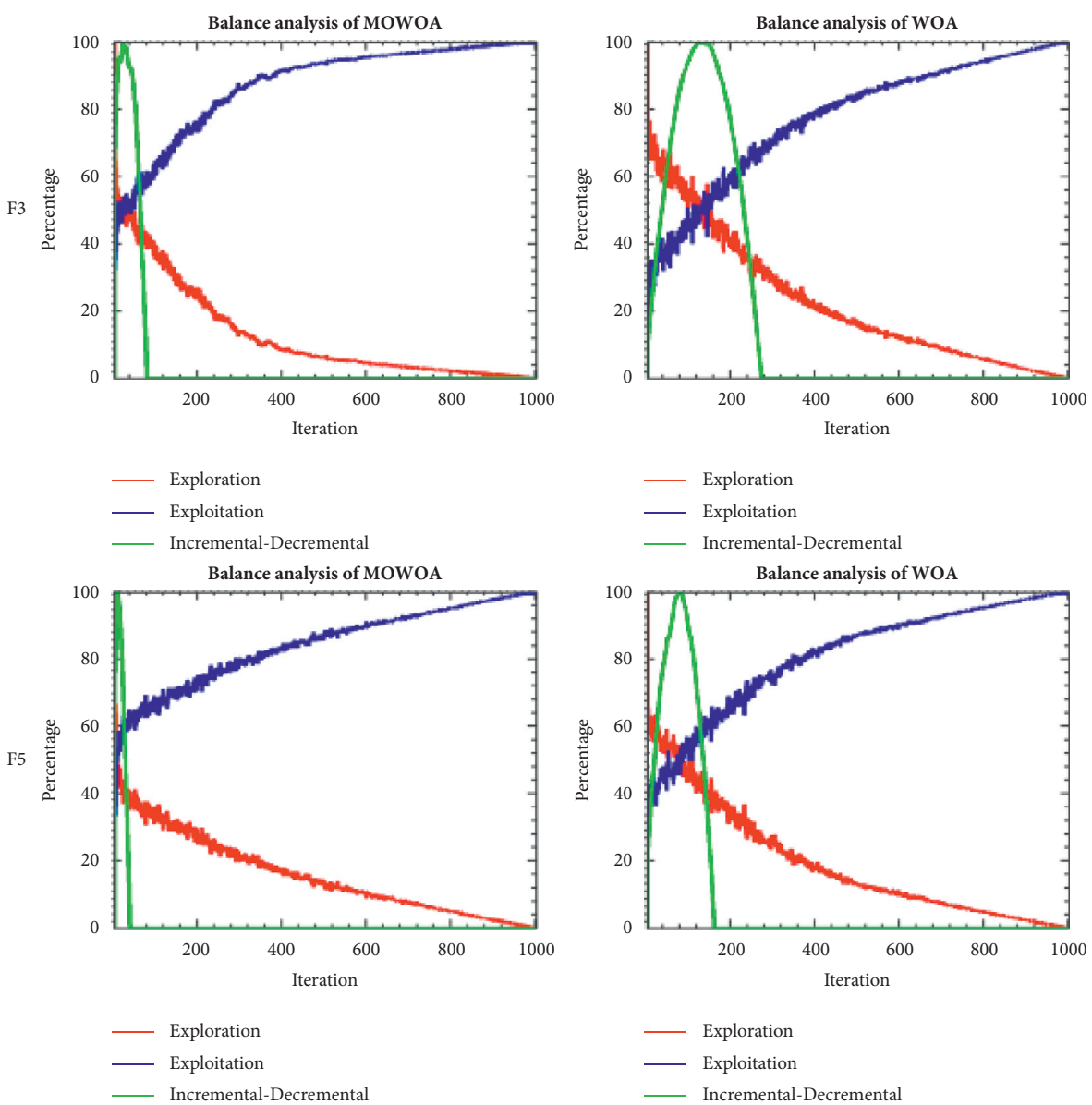

(a)

Figure 3: Continued. 

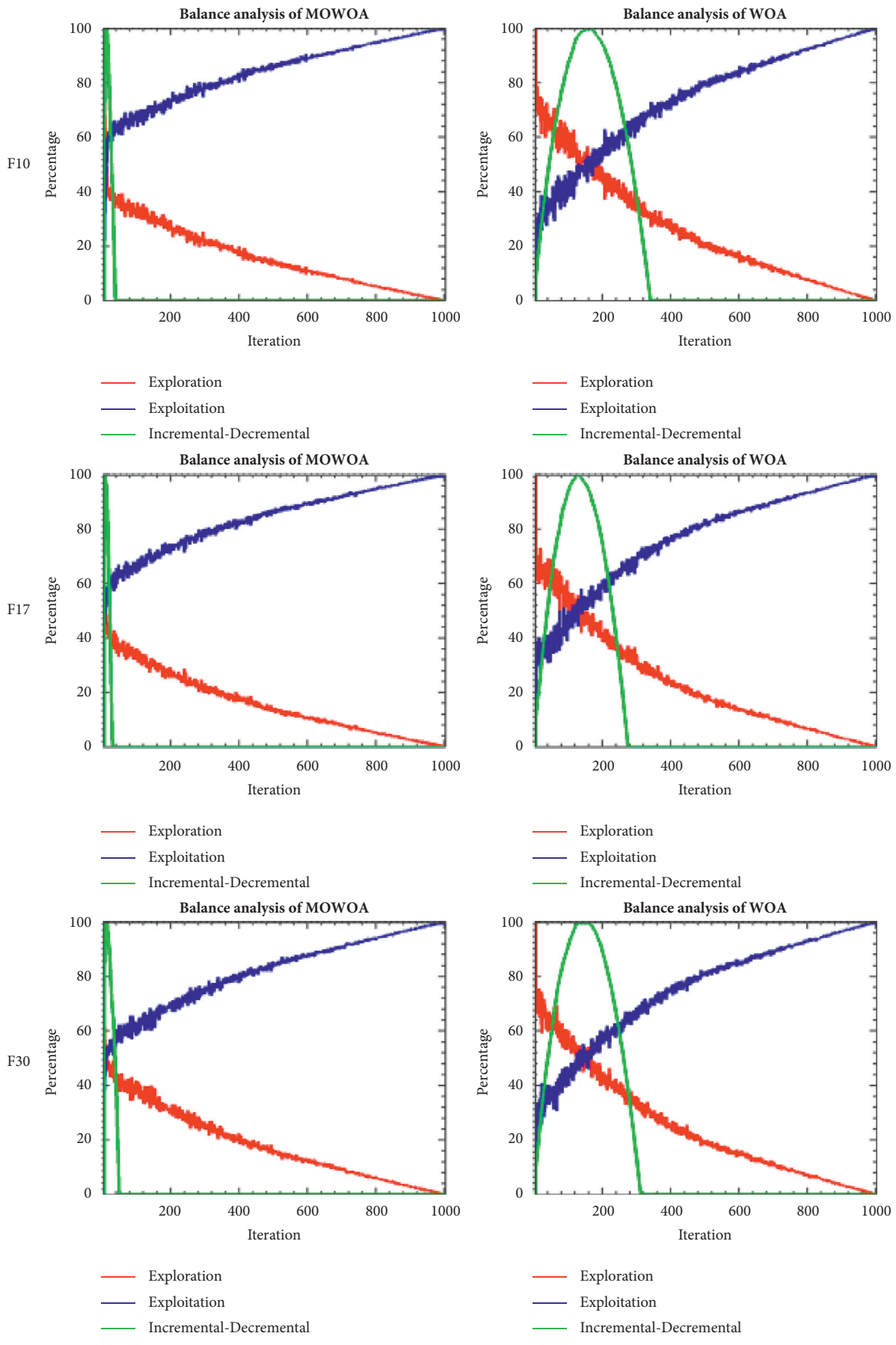

(b)

FIGURE 3: Balance analysis of algorithms.

On the Unimodal functions, the performance of MEWOA can be ranked in the middle among the listed algorithms; especially, for the F2 and F3 functions, MEWOA can be ranked third among all algorithms, exceeding the classical algorithm $\mathrm{DE}$, so the overall results are still good.

On the Multimodal functions, MEWOA does not perform as well as on the Unimodal function, in terms of both convergence speed and convergence accuracy. However, on F10, its results are still relatively good. The final convergence accuracy can be ranked third. In addition, according to the picture, except for MFO, MEWOA can achieve a good convergence effect in the first half process iteration.

MEWOA has the best results on Hybrid functions, especially on F13, F16, F18, and F19, where MEWOA can be ranked second among all functions. According to the experimental results, it can also be found that the difference between the convergence accuracy of MEWOA and the firstranked algorithm is not very large in the remaining functions. 
F1

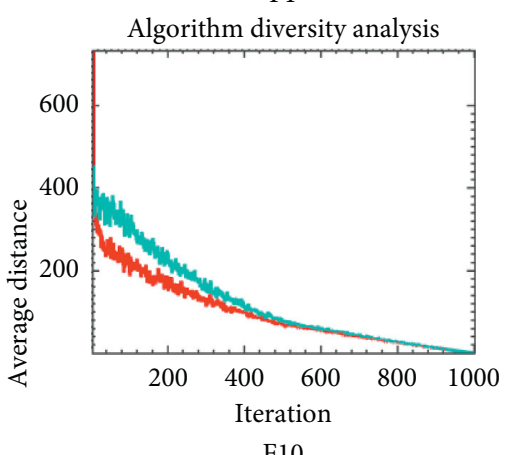

F10

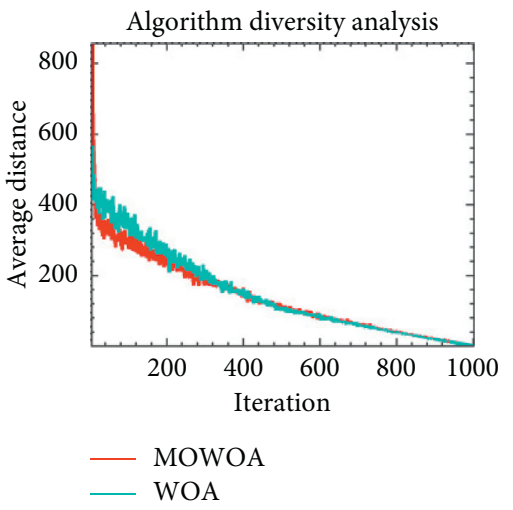

F3

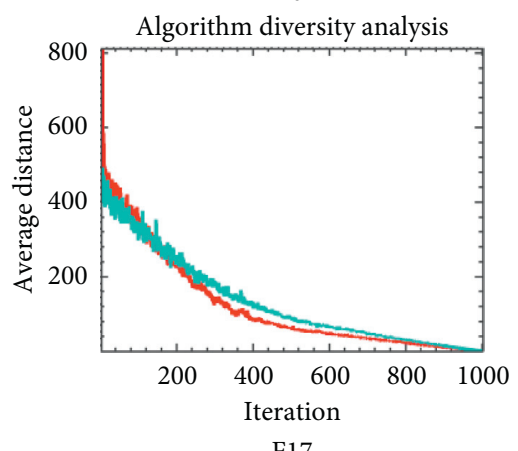

F17

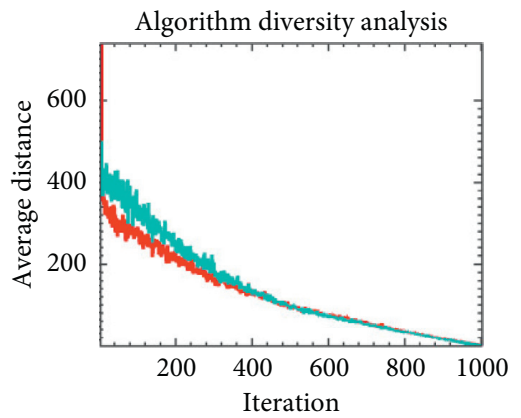

F5

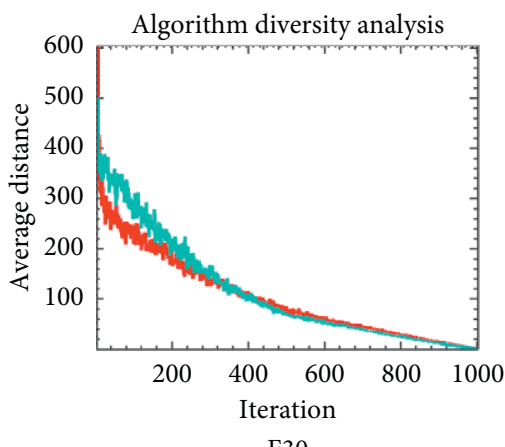

F30

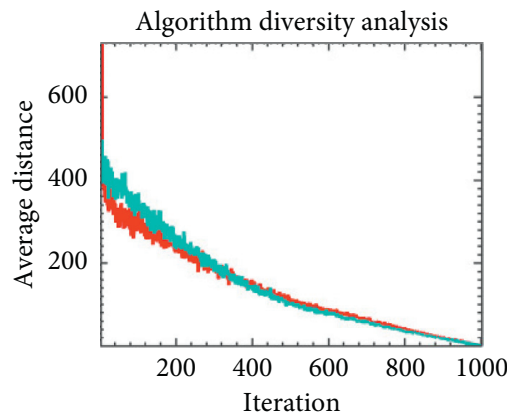

FIgURE 4: Diversity analysis of algorithms.

TABLE 3: Benchmark functions utilized in the scalability experiment.

\begin{tabular}{|c|c|c|c|c|c|c|c|c|c|}
\hline Dim & & & MEWOA & OBWOA & ACWOA & BWOA & BMWOA & CCMWOA & CWOA \\
\hline \multirow{4}{*}{$\mathrm{F} 1$} & \multirow{2}{*}{50} & AVG & $1.0377 E+04$ & $7.6546 E+09$ & $5.1362 E+09$ & $2.9030 E+08$ & $3.3981 E+08$ & $2.2425 E+10$ & $2.6554 E+09$ \\
\hline & & STD & $1.4448 E+04$ & $3.7797 E+09$ & $2.1333 E+09$ & $1.8009 E+08$ & $1.7216 E$ & 5.770 & 2.43 \\
\hline & \multirow{2}{*}{100} & AVG & $3.4074 E+04$ & $6.1323 E+09$ & $3.8614 E+09$ & $3.5406 E+$ & $6.4327 E$ & & \\
\hline & & STD & $1.4310 E+$ & $3.9653 E+$ & $1.6506 E+09$ & $2.4011 E+$ & $3.1031 E+$ & $3.8734 E$ & $1.7015 E+09$ \\
\hline \multirow{4}{*}{ F2 } & \multirow{2}{*}{50} & AVG & $8.1668 E+15$ & $3.0916 E+35$ & $1.4866 E+38$ & $4.2055 E+27$ & $1.1327 E+26$ & $6.9725 E+39$ & $1.8395 E+33$ \\
\hline & & STD & & 1.667 & & 1.548 & 5.412 & & 9.466 \\
\hline & \multirow{2}{*}{100} & AVG & 5.48 & 2.620 & 6.232 & 3.141 & 3.173 & & 4.915 \\
\hline & & STD & $1.5236 E+13$ & $7.3949 E+32$ & $3.1390 E+36$ & $1.6248 E+26$ & $8.8369 E+20$ & 9.43 & $2.6657 E+33$ \\
\hline \multirow{4}{*}{ F3 } & \multirow{2}{*}{50} & AVG & $7.4126 E+03$ & $5.0041 E+04$ & $5.3069 E+04$ & $5.8975 E+04$ & $7.2088 E+04$ & 7.76 & $1.7950 E+05$ \\
\hline & & STD & 3 & 7.56 & 1.1 & 9.41 & 9.8 & & 5.8 \\
\hline & \multirow{2}{*}{100} & AVG & 2.54 & 4.69 & & 5.13 & 6.07 & 7.48 & 1.42 \\
\hline & & STD & 3.33 & 1.216 & 1.143 & 1.37 & 9.02 & 6.3 & $4.3977 E+04$ \\
\hline \multirow{4}{*}{$\mathrm{F} 4$} & \multirow{2}{*}{50} & AVG & $5.1424 E+02$ & 1.310 & 1.509 & 6.06 & 6.34 & 3.4 & $7.2047 E+02$ \\
\hline & & STD & $5.0383 E+01$ & 9.237 & 9.117 & 4.40 & 5.30 & & $1.9633 E+02$ \\
\hline & \multirow{2}{*}{100} & AVG & $5.0761 E+02$ & $1.0433 E+$ & $9.3826 E$ & $5.6889 E$ & 5.677 & 2.8 & $6.1254 E+02$ \\
\hline & & STD & & 5.82 & & & & & $1.0315 E+02$ \\
\hline \multirow{4}{*}{ F5 } & \multirow[b]{2}{*}{50} & AVG & & $80334 F+$ & $8.0383 E+02$ & $7.6877 E+02$ & $7.9177 E+02$ & -02 & $7.9951 E+02$ \\
\hline & & & & & & & & & $6.2931 E+01$ \\
\hline & \multirow{2}{*}{100} & AVG & $6.7717 E+02$ & $7.9697 E+02$ & $7.8954 E+02$ & $7.5788 E+02$ & $7.7647 E+02$ & $8.1562 E+02$ & $8.2434 E+02$ \\
\hline & & STD & $3.3853 E+01$ & $2.7023 E+01$ & $2.6015 E+01$ & $3.4720 E+01$ & $4.7106 E+01$ & $3.5426 E+01$ & $6.2786 E+01$ \\
\hline \multirow{4}{*}{ F6 } & \multirow[b]{2}{*}{50} & AVG & $6.4280 E+02$ & $6.6924 E+02$ & $6.6517 E+02$ & $6.6566 E+02$ & $6.6621 E+02$ & $6.7035 E+02$ & $6.7652 E+02$ \\
\hline & & STD & $1.0910 E+01$ & $6.4989 E+00$ & $4.5122 E+00$ & $8.3904 E+00$ & $8.9785 E+00$ & $8.5912 E+00$ & $1.2226 E+01$ \\
\hline & \multirow[b]{2}{*}{100} & AVG & $6.3798 E+02$ & $6.6650 E+02$ & $6.6353 E+02$ & $6.6470 E+02$ & $6.6466 E+02$ & $6.7030 E+02$ & $6.7069 E+02$ \\
\hline & & STD & $1.1490 E+01$ & $6.1196 E+00$ & $6.8462 E+00$ & $8.2608 E+00$ & $9.3215 E+00$ & $8.2339 E+00$ & $1.1780 E+01$ \\
\hline
\end{tabular}


TABle 3: Continued.

\begin{tabular}{|c|c|c|c|c|c|c|c|c|c|}
\hline Dim & & & MEWOA & OBWOA & ACWOA & BWOA & BMWOA & CCMWOA & CWOA \\
\hline \multirow{4}{*}{ F7 } & \multirow{2}{*}{50} & $\mathrm{AVG}$ & $9.6655 E+02$ & $1.2959 E+03$ & $1.2357 E+03$ & $1.2617 E+03$ & $1.2439 E+03$ & $1.2902 E+03$ & $1.2742 E+03$ \\
\hline & & STD & $7.1608 E+01$ & $4.4520 E+01$ & $6.1102 E+01$ & $7.9393 E+01$ & $9.3492 E+01$ & $6.4993 E+01$ & $7.4210 E+01$ \\
\hline & \multirow{2}{*}{100} & AVG & $9.5751 E+02$ & $1.2807 E+03$ & $1.2236 E+03$ & $1.2400 E+03$ & $1.1750 E+03$ & $1.2622 E+03$ & $1.2446 E+03$ \\
\hline & & STD & $5.9145 E+01$ & $5.9135 E+01$ & $6.1447 E+01$ & $7.5216 E+01$ & $1.0336 E+02$ & $8.1953 E+01$ & $6.7215 E+01$ \\
\hline \multirow{4}{*}{ F8 } & \multirow{2}{*}{50} & AVG & $1.0013 E+03$ & $1.0045 E+03$ & $1.0177 E+03$ & $9.8480 E+02$ & $1.0180 E+03$ & $1.0456 E+03$ & $1.0319 E+03$ \\
\hline & & STD & $5.5037 E+01$ & $2.5078 E+01$ & $2.6427 E+01$ & $2.7098 E+01$ & $2.4785 E+01$ & $2.7515 E+01$ & $4.2507 E+01$ \\
\hline & \multirow{2}{*}{100} & AVG & $9.7555 E+02$ & $1.0016 E+03$ & $1.0081 E+03$ & $9.7816 E+02$ & $1.0067 E+03$ & & $1.0347 E+03$ \\
\hline & & STD & $4.4852 E+01$ & $2.4995 E+01$ & $1.9787 E+01$ & $1.7740 E+01$ & $3.4795 E+01$ & $3.0539 E+01$ & $5.6612 E+01$ \\
\hline \multirow{4}{*}{ F9 } & \multirow[b]{2}{*}{50} & $\mathrm{AVG}$ & $5.8348 E+03$ & $7.3478 E+03$ & $7.1516 E+03$ & $6.2441 E+03$ & $7.8921 E+03$ & $7.9590 E+03$ & $8.8671 E+03$ \\
\hline & & STD & $1.9274 E+03$ & $1.1299 E+03$ & $9.7706 E+02$ & $8.7146 E+02$ & $1.3587 E+03$ & $1.4096 E+03$ & $2.5451 E+03$ \\
\hline & \multirow{2}{*}{100} & $\mathrm{AVG}$ & $6.0388 E+03$ & $6.8714 E+03$ & $6.8865 E+03$ & $5.8989 E+03$ & $6.9697 E+03$ & $7.5847 E+03$ & $8.1388 E+03$ \\
\hline & & STD & $2.1690 E+03$ & $9.1811 E+02$ & $9.7778 E+02$ & $4.5234 E+02$ & $1.0148 E+03$ & $1.0859 E+03$ & $2.5020 E+03$ \\
\hline \multirow{4}{*}{ F10 } & \multirow{2}{*}{50} & $\mathrm{AVG}$ & $5.2132 E+03$ & $6.3611 E+03$ & $6.6167 E+03$ & $6.7578 E+03$ & $7.7359 E+03$ & $7.0624 E+03$ & $6.0753 E+03$ \\
\hline & & STD & $E+02$ & +02 & 9.17 & 9.45 & & & $E+02$ \\
\hline & \multirow{2}{*}{100} & AVG & $5.3579 E+03$ & $6.1734 E+03$ & $6.2137 E+03$ & $6.3582 E+03$ & $7.0292 E+03$ & $6.9010 E+03$ & $5.9492 E+03$ \\
\hline & & STD & $8.1114 E+02$ & $6.1867 E+02$ & $8.4074 E+02$ & $8.1917 E+02$ & $8.3520 E+02$ & $39 E+02$ & $6.1961 E+02$ \\
\hline \multirow{4}{*}{ F11 } & \multirow{2}{*}{50} & AVG & & & & & & & $5263 E+03$ \\
\hline & & STD & $E+01$ & $4 E+03$ & $E+02$ & $E+02$ & $6 E+02$ & & $1.6474 E+03$ \\
\hline & \multirow{2}{*}{100} & AVG & $1.3139 E+03$ & $2.3188 E+03$ & $E+03$ & 1.55 & $E+03$ & 3.0 & $E+03$ \\
\hline & & STD & $6.5062 E+01$ & $1.0241 E+03$ & $7.5378 E+02$ & 1.35 & $8 E+02$ & 4.66 & $5 E+03$ \\
\hline \multirow{4}{*}{ F12 } & & $\mathrm{AVG}$ & $2.0866 E+06$ & $5.4663 E+08$ & $7.0549 E+08$ & $1.3443 E+08$ & $1.0153 E+08$ & $3.1251 E+09$ & $2.1403 E+08$ \\
\hline & 50 & STD & +06 & $E+08$ & $E+$ & 7 & 5. & & $E+08$ \\
\hline & & AVG & $E+06$ & $E+08$ & $E+$ & & 4.3 & & $7.3297 E+07$ \\
\hline & & STD & $1.1317 E+06$ & $5.2068 E+08$ & $3.6694 E+08$ & $3.6693 E+07$ & $2.7375 E+07$ & $8.8761 E+08$ & $6.9963 E+07$ \\
\hline & & $\mathrm{AVG}$ & $2.8736 E+04$ & $3.2546 E+05$ & $5.8098 E+07$ & $3.1410 E+05$ & $5.3145 E+05$ & $1.7827 E+08$ & $1.5088 E+05$ \\
\hline & 5 & STD & $2.0176 E+04$ & $3.3803 E+05$ & $E+08$ & $E+05$ & $E+05$ & $E+08$ & $4 E+04$ \\
\hline F13 & & $\mathrm{AVG}$ & $2.8030 E+04$ & $9.3162 E+06$ & $1.1670 E+08$ & $1.6722 E+05$ & $1.7872 E+05$ & $3.7303 E+07$ & $1.4285 E+05$ \\
\hline & 100 & STD & $2.4067 E+04$ & $4.9866 E+07$ & & & $1.2190 E+05$ & $8.7842 E+07$ & $9.8340 E+04$ \\
\hline & 5 & $\mathrm{AVG}$ & & & & & & & \\
\hline & 5 & STD & +04 & +06 & 5 & & 05 & & $2 E+06$ \\
\hline $\mathrm{F}$ & & $\mathrm{AVG}$ & $2.7654 E+04$ & $8.1587 E+05$ & $6 E+05$ & $E+06$ & $E+05$ & $E+06$ & $56 E+05$ \\
\hline & & STD & $2.1704 E+04$ & $5.6323 E+05$ & & & $5 E+05$ & & $81 E+05$ \\
\hline & & AVG & $1.9648 E+04$ & $1.9513 E+06$ & $1.0018 E+07$ & $2.3326 E+05$ & $1.2765 E+05$ & $4.7314 E+06$ & $6.6735 E+06$ \\
\hline & & STD & & & & & & & $4 E+07$ \\
\hline & & AVG & $8 E+04$ & $E+06$ & $E+07$ & $E+04$ & $8 E+04$ & & $1.9551 E+06$ \\
\hline & & STD & $1.2602 E+04$ & $1.0657 E+07$ & $3.8329 E+07$ & $7.1976 E+04$ & $2.8165 E+04$ & $3.9738 E+06$ & $4.3858 E+06$ \\
\hline & & $\mathrm{AVG}$ & $2.6782 E+03$ & $3.9833 E+03$ & $3.9266 E+03$ & $3.9609 E+03$ & $3.4721 E+03$ & $4.3136 E+03$ & $3.7458 E+03$ \\
\hline & & STD & $3.7601 E+02$ & $5.5297 E+02$ & $3 E+02$ & $E+02$ & $E+02$ & $E+02$ & $9 E+02$ \\
\hline $\mathrm{F}$ & & $\mathrm{AVG}$ & $2.6047 E+03$ & $3.6881 E+03$ & & & $681 E+03$ & & $3.6832 E+03$ \\
\hline & 100 & STD & $2.8196 E+02$ & $5.4792 E+02$ & $2.9038 E+02$ & $5.2779 E+02$ & $4.2993 E+02$ & $4.4155 E+02$ & $5.0216 E+02$ \\
\hline & 50 & $\mathrm{AVG}$ & $2.3075 E+03$ & $2.6205 E+03$ & $2.6403 E+03$ & $2.6899 E+03$ & $2.5231 E+03$ & $2.7808 E+03$ & $2.6261 E+03$ \\
\hline & 5 & STD & +02 & +02 & & & & & $2.3234 E+02$ \\
\hline & & $\mathrm{AVG}$ & $2.3092 E+03$ & $2.5878 E+03$ & $2.5663 E+03$ & $2.5445 E+03$ & $2.3901 E+03$ & $2.6705 E+03$ & $2.5358 E+03$ \\
\hline & 100 & STD & $2.2490 E+02$ & $2.3905 E+02$ & $1.8353 E+02$ & $3.2721 E+02$ & $2.1330 E+02$ & $3.9669 E+02$ & $2.7897 E+02$ \\
\hline & & $\mathrm{AVG}$ & $4.8531 E+05$ & $2.7178 E+06$ & $4.9753 E+06$ & $4.7870 E+06$ & $3.9696 E+06$ & $1.0008 E+07$ & $5.2516 E+06$ \\
\hline & 5 & STD & $2 E+05$ & $7 E+$ & 4. & 3. & $4.1710 E+06$ & $E+06$ & $5.2077 E+06$ \\
\hline F18 & & AVG & $3.2319 E+05$ & $24 E+06$ & $2.0466 E+06$ & $3.4255 E+06$ & $3.0443 E+06$ & $8.7689 E+06$ & $3.2644 E+06$ \\
\hline & & STD & $2.5201 E+05$ & $2.2250 E+06$ & $3.0810 E+06$ & $2.9805 E+06$ & $3.7768 E+06$ & $9.9315 E+06$ & $3.9346 E+06$ \\
\hline & & $\mathrm{AVG}$ & $2.0880 E+04$ & $1.1253 E+06$ & $1.4096 E+07$ & $4.5512 E+06$ & $8.4651 E+05$ & $4.9944 E+06$ & $2.4743 E+06$ \\
\hline & & STD & $2.0800 E+04$ & $9.3945 E+05$ & $2.3132 E+07$ & $3.8997 E+06$ & $8.9808 E+05$ & $4.0455 E+06$ & $2.5119 E+06$ \\
\hline $\mathrm{F}$ & & AVG & $2.0597 E+04$ & $1.1714 E+06$ & $1.4314 E+07$ & $3.0040 E+06$ & $2.9342 E+05$ & $3.3380 E+06$ & $5.8437 E+06$ \\
\hline & 100 & STD & $1.9606 E+04$ & $9.2106 E+05$ & $2.5721 E+07$ & $2.8549 E+06$ & $3.0967 E+05$ & $4.0676 E+06$ & $2.1544 E+07$ \\
\hline & & $\mathrm{AVG}$ & $2.5745 E+03$ & $2.7246 E+03$ & $2.6898 E+03$ & $2.7568 E+03$ & $2.7300 E+03$ & $2.7067 E+03$ & $2.8540 E+03$ \\
\hline & 5 & STD & $2.1430 E+02$ & $2.3025 E+02$ & $1.3537 E+02$ & $1.9178 E+02$ & $1.8621 E+02$ & $2.1710 E+02$ & $2.0568 E+02$ \\
\hline & & AVG & $2.4905 E+03$ & $2.7821 E+03$ & $2.6152 E+03$ & $2.6534 E+03$ & $2.6362 E+03$ & $2.6471 E+03$ & $2.7217 E+03$ \\
\hline & & STD & $1.7572 E+02$ & $1.6627 E+02$ & $1.4973 E+02$ & $1.7623 E+02$ & $1.7958 E+02$ & $1.7418 E+02$ & $1.8903 E+02$ \\
\hline
\end{tabular}


TABle 3: Continued.

\begin{tabular}{|c|c|c|c|c|c|c|c|c|c|}
\hline Dim & & & MEWOA & OBWOA & ACWOA & BWOA & BMWOA & CCMWOA & CWOA \\
\hline \multirow{4}{*}{ F21 } & \multirow{2}{*}{50} & AVG & $2.4895 E+03$ & $2.5943 E+03$ & $2.5948 E+03$ & $2.5885 E+03$ & $2.5353 E+03$ & $2.6227 E+03$ & $2.5933 E+03$ \\
\hline & & STD & $3.8874 E+01$ & $.1381 E+01$ & $3573 E+01$ & $5.8174 E+01$ & $4.7024 E+01$ & & 6.23 \\
\hline & \multirow{2}{*}{100} & AVG & $2.4743 E+03$ & $2.5761 E+03$ & $2.5755 E+03$ & $2.5653 E+03$ & $2.5220 E+03$ & $2.6198 E+03$ & $2.5813 E+03$ \\
\hline & & STD & & $8.4792 E+01$ & $3.2601 E+01$ & $5.3228 E+01$ & $4.5418 E+01$ & $4.8100 E+01$ & $6.8689 E+01$ \\
\hline \multirow{4}{*}{ F22 } & \multirow{2}{*}{50} & AVG & $6.1490 E+03$ & $3173 E+03$ & $5.4178 E+03$ & $6.3144 E+03$ & $5.1290 E+03$ & $7.4041 E+03$ & $6.7333 E+03$ \\
\hline & & STD & $1.9016 E+03$ & $1.5471 E+03$ & $2.0351 E+03$ & $2.5268 E+03$ & & & $2.1379 E+03$ \\
\hline & \multirow{2}{*}{100} & AVG & $6.3054 E+03$ & $6.7626 E+03$ & & 7.400 & & & \\
\hline & & STD & $1.0591 E+03$ & $1.9153 E+03$ & $1.7276 E+$ & $1.3291 E+03$ & $2.4923 E$ & $1.2612 E$ & $1.9830 E+03$ \\
\hline \multirow{4}{*}{ F23 } & \multirow[b]{2}{*}{50} & AVG & $2.8555 E+03$ & $3.0652 E+03$ & & $3.0740 E+03$ & & & $3.1005 E+03$ \\
\hline & & STD & & 9.9 & & 1.12 & & & $1.0485 E+02$ \\
\hline & \multirow{2}{*}{100} & $\mathrm{AVG}$ & $E+03$ & 3.132 & $E+03$ & $3.0578 E+03$ & & & $3.0590 E+03$ \\
\hline & & STD & $6.7638 E+01$ & $1.0696 E+02$ & $5.5154 E+01$ & $1.0289 E+02$ & $8.3256 E+01$ & $9.6440 E+01$ & $1.0534 E+02$ \\
\hline \multirow{4}{*}{$\mathrm{F} 24$} & \multirow{2}{*}{50} & $\mathrm{AVG}$ & $3.0137 E+03$ & $3.2187 E+03$ & $3.1779 E+03$ & $3.1921 E+03$ & $31348 E+03$ & $3.3436 E+03$ & $3.2694 E+03$ \\
\hline & & STD & 1 & 1.41 & 1 & 9.86 & & & \\
\hline & \multirow{2}{*}{100} & AVG & $E+03$ & $E+03$ & $E+03$ & 3.18 & & & $E+03$ \\
\hline & & STD & 5.960 & 1.09 & & 7.7 & & & \\
\hline \multirow{4}{*}{ F25 } & \multirow{2}{*}{50} & & & & & & & & \\
\hline & & STD & & 2 & & 4.1 & & & \\
\hline & \multirow{2}{*}{100} & AVG & $2.9113 E+03$ & $3.0928 E+03$ & 3.1 & 2.99 & 2. & 3. & 3.0 \\
\hline & & STD & $2.2493 E+01$ & & & & & & \\
\hline \multirow{4}{*}{ F26 } & \multirow{2}{*}{50} & AVG & & & & & & & \\
\hline & & & & & & & & & \\
\hline & \multirow{2}{*}{100} & AVG & +03 & +03 & $E+03$ & $E+03$ & & & $4 E+03$ \\
\hline & & STD & $5.3520 E+02$ & $1.2603 E+03$ & $7.4569 E+02$ & $1.6258 E+03$ & $6 E+03$ & $E+03$ & $1.0925 E+03$ \\
\hline \multirow{4}{*}{ F27 } & \multirow{2}{*}{50} & AVG & $3.5106 E+03$ & $3.4028 E+03$ & $34750 F+03$ & $3.4213 E+03$ & & & $3.3903 E+03$ \\
\hline & & STD & $E+02$ & 2 & 2 & 1.27 & & & \\
\hline & \multirow{2}{*}{100} & AVG & $E+03$ & & & & & & $2 E+03$ \\
\hline & & STD & $E+02$ & $8.9512 E+01$ & $8.9662 E+01$ & $8.8219 E+01$ & $3.6244 E$ & $1.3092 E$ & $9.3400 E+01$ \\
\hline \multirow{4}{*}{ F28 } & \multirow{2}{*}{50} & AVG & & & & & & & \\
\hline & & STD & & & $2.0019 E+02$ & & & & $2.3404 E+02$ \\
\hline & \multirow{2}{*}{100} & AVG & $8.2899 E+03$ & $3.7758 E+03$ & & & & & \\
\hline & & STD & $1.0768 E+03$ & $2.6586 E+02$ & +02 & $3.8695 E$ & 1 & & $9.2971 E+01$ \\
\hline & & AVG & $4.1814 E+03$ & $4.7997 E+03$ & $4.7766 E+03$ & $5.0867 E+03$ & $4.8669 E+03$ & $5.2353 E+03$ & $4.9032 E+03$ \\
\hline & 5 & STD & & & & & & & $4.6297 E+02$ \\
\hline & & AVG & $4.0297 E+03$ & $4.6450 E+03$ & $4.5318 E+03$ & $4.9508 E+03$ & $4.6385 E+03$ & $5.2996 E+03$ & $4.8376 E+03$ \\
\hline & & STD & $2.6601 E+02$ & $3.6948 E+02$ & $2.8181 E+02$ & $3.1316 E+02$ & $3.0809 E+02$ & $6.6241 E+02$ & $4.7446 E+02$ \\
\hline & & $\mathrm{AVG}$ & $3.0309 E+04$ & $4.2253 E+07$ & $6.2921 E+07$ & $2.1406 E+07$ & $6.6288 E+06$ & $8.3127 E+07$ & $1.2808 E+07$ \\
\hline & 5 & STD & $2.2691 E+04$ & $1.0790 E+08$ & & $2.4931 E+07$ & & $9.1773 E+07$ & $1.3470 E+07$ \\
\hline & & AVG & $1.7144 E+04$ & $5.9401 E+07$ & $4.2420 E+07$ & $1.6012 E+07$ & $4.4775 E+06$ & $5.1449 E+07$ & $1.1866 E+07$ \\
\hline & & STD & $7.9091 E+03$ & $1.7815 E+08$ & $3.1937 E+07$ & $1.3827 E+07$ & $3.6919 E+06$ & $3.8182 E+07$ & $6.9753 E+06$ \\
\hline
\end{tabular}

Finally, in the Composition functions, we can see that the results shown by MEWOA are still good in the convergence graphs of both F22 and F30 functions, especially in the F22 function, which can achieve a better solution than the other algorithms.

4.6. Comparison with Advanced Algorithms. To further verify the performance of MEWOA, this section has selected some advanced algorithms to compare with MEWOA. Among the compared algorithms, champion algorithms, such as LSHADE, are included, as well as improved algorithms on DE, such as SADE, and algorithms with stronger performance, such as HCLPSO. The specific algorithms involved in the comparison are shown as follows. (i) Adaptive DE with success-history and linear population size reduction (HCLPSO) [119]

(ii) Self-adaptive differential evolution (SADE) [120]

(iii) Adaptive differential evolution with optional external archive (JADE) [121]

(iv) Comprehensive learning particle swarm optimizer (CLPSO) [122]

(v) Adaptive DE with success-history and linear popuation size reduction (LSHADE) [123]

(vi) LSHADE_cnEpSi (LSHADE_ES) [124].

(vii) Multistrategy enhanced sine cosine algorithm (MSCA) [16]

The experimental parameters were set in the same way as in the previous section, and in addition, we have modified 
TABLE 4: Comparison results of different peers.

\begin{tabular}{|c|c|c|c|c|c|c|c|c|}
\hline & & MEWOA & OBWOA & ACWOA & BWOA & BMWOA & CCMWOA & CWOA \\
\hline \multirow{4}{*}{ F1 } & AVG & $1.1469 E+05$ & $9.7743 E+09$ & $7.8058 E+09$ & $9.6529 E+08$ & $1.0985 E+09$ & $2.4867 E+10$ & $4.0000 E+09$ \\
\hline & STD & $4.1160 E+05$ & $4.1822 E+09$ & $2.8798 E+09$ & $6.1575 E+08$ & $5.0173 E+08$ & $5.0888 E+09$ & $2.8487 E+09$ \\
\hline & Rank & 1 & 6 & 5 & 2 & 3 & 7 & 4 \\
\hline & $p$ value & & $1.7344 E-06$ & $1.7344 E-06$ & $1.7344 E-06$ & $1.7344 E-06$ & $1.7344 E-06$ & $1.7344 E-06$ \\
\hline \multirow{4}{*}{$\mathrm{F} 2$} & AVG & $8.1708 E+15$ & $6.2853 E+34$ & $9.2862 E+34$ & $7.1393 E+30$ & $1.0002 E+27$ & $1.9200 E+41$ & $1.0852 E+34$ \\
\hline & STD & $4.4709 E+16$ & $2.4742 E+35$ & $3.2104 E+35$ & $2.7272 E+31$ & $3.4542 E+27$ & $9.9696 E+41$ & $4.5433 E+34$ \\
\hline & Rank & 1 & 5 & 6 & 3 & 2 & 7 & 4 \\
\hline & $p$ value & & $1.7344 E-06$ & $1.7344 E-06$ & $1.7344 E-06$ & $1.7344 E-06$ & $1.7344 E-06$ & $1.7344 E-06$ \\
\hline \multirow{4}{*}{ F3 } & AVG & $1.3261 E+04$ & $5.8532 E+04$ & $5.3179 E+04$ & $6.7334 E+04$ & $8.1538 E+04$ & $7.8703 E+04$ & $1.9351 E+05$ \\
\hline & STD & $7.4562 E+03$ & $7.9256 E+03$ & $9.6568 E+03$ & $9.6164 E+03$ & $6.7204 E+03$ & $6.4792 E+03$ & $4.4523 E+04$ \\
\hline & Rank & 1 & 3 & 2 & 4 & 6 & 5 & 7 \\
\hline & $p$ value & & $1.7344 E-06$ & $1.7344 E-06$ & $1.7344 E-06$ & $1.7344 E-06$ & $1.7344 E-06$ & $1.7344 E-06$ \\
\hline \multirow{4}{*}{$\mathrm{F} 4$} & AVG & $5.1430 E+02$ & $1.6724 E+03$ & $1.4835 E+03$ & $7.1171 E+02$ & $7.3454 E+02$ & $5.0608 E+03$ & $1.0173 E+03$ \\
\hline & STD & $3.8031 E+01$ & $9.9516 E+02$ & $7.3853 E+02$ & $1.3421 E+02$ & $1.0306 E+02$ & $1.3683 E+03$ & $4.7652 E+02$ \\
\hline & Rank & 1 & 6 & 5 & 2 & 3 & 7 & 4 \\
\hline & $p$ value & & $1.7344 E-06$ & $1.7344 E-06$ & $1.7344 E-06$ & $1.7344 E-06$ & $1.7344 E-06$ & $1.7344 E-06$ \\
\hline \multirow{4}{*}{ F5 } & AVG & $6.8487 E+02$ & $8.1524 E+02$ & $8.1331 E+02$ & $7.7250 E+02$ & $8.0978 E+02$ & $8.4433 E+02$ & $8.1605 E+02$ \\
\hline & STD & $4.7570 E+01$ & $3.3664 E+01$ & $2.4957 E+01$ & $4.0902 E+01$ & $4.8970 E+01$ & $3.4510 E+01$ & $5.3895 E+01$ \\
\hline & Rank & 1 & 5 & 4 & 2 & 3 & 7 & 6 \\
\hline & $p$ value & & $2.1266 E-06$ & $1.9209 E-06$ & $2.3704 E-05$ & $2.6033 E-06$ & $1.7344 E-06$ & $2.1266 E-06$ \\
\hline \multirow{4}{*}{ F6 } & AVG & $6.4282 E+02$ & $6.7097 E+02$ & $6.7025 E+02$ & $6.6603 E+02$ & $6.6566 E+02$ & $6.7218 E+02$ & $6.7673 E+02$ \\
\hline & STD & $1.4507 E+01$ & $5.7482 E+00$ & $7.7197 E+00$ & $7.1928 E+00$ & $9.7165 E+00$ & $6.8770 E+00$ & $1.4458 E+01$ \\
\hline & Rank & 1 & 5 & 4 & 3 & 2 & 6 & 7 \\
\hline & $p$ value & & $1.9209 E-06$ & $2.1266 E-06$ & $7.6909 E-06$ & $2.8434 E-05$ & $2.1266 E-06$ & $4.2857 E-06$ \\
\hline \multirow{4}{*}{ F7 } & AVG & $9.6141 E+02$ & $1.3112 E+03$ & $1.2484 E+03$ & $1.2622 E+03$ & $1.2408 E+03$ & $1.3043 E+03$ & $1.2987 E+03$ \\
\hline & STD & $5.5626 E+01$ & $6.2946 E+01$ & $6.3206 E+01$ & $7.4289 E+01$ & $1.0208 E+02$ & $6.1390 E+01$ & $8.7682 E+01$ \\
\hline & Rank & 1 & 7 & 3 & 4 & 2 & 6 & 5 \\
\hline & $p$ value & & $1.7344 E-06$ & $1.7344 E-06$ & $1.7344 E-06$ & $1.7344 E-06$ & $1.7344 E-06$ & $1.7344 E-06$ \\
\hline \multirow{4}{*}{ F8 } & $\mathrm{AVG}$ & $9.7134 E+02$ & $1.0202 E+03$ & $1.0168 E+03$ & $1.0079 E+03$ & $1.0273 E+03$ & $1.0640 E+03$ & $1.0492 E+03$ \\
\hline & STD & $4.7508 E+01$ & $2.8426 E+01$ & $2.8267 E+01$ & $2.9916 E+01$ & $3.5155 E+01$ & $2.7126 E+01$ & $4.4532 E+01$ \\
\hline & Rank & 1 & 4 & 3 & 2 & 5 & 7 & 6 \\
\hline & $p$ value & & $7.1570 E-04$ & $3.5888 E-04$ & $1.1973 E-03$ & $3.0650 E-04$ & $3.1817 E-06$ & $1.7988 E-05$ \\
\hline \multirow{4}{*}{ F9 } & $\mathrm{AVG}$ & $5.3008 E+03$ & $7.7529 E+03$ & $7.6542 E+03$ & $6.9325 E+03$ & $8.1060 E+03$ & $8.3760 E+03$ & $9.4107 E+03$ \\
\hline & STD & $2.3047 E+03$ & $1.3121 E+03$ & $1.3167 E+03$ & $1.2491 E+03$ & $1.2116 E+03$ & $1.1790 E+03$ & $3.4607 E+03$ \\
\hline & Rank & 1 & 4 & 3 & 2 & 5 & 6 & 7 \\
\hline & $p$ value & & $5.3070 E-05$ & $2.6134 E-04$ & $4.3896 E-03$ & $5.7924 E-05$ & $2.5967 E-05$ & $7.6909 E-06$ \\
\hline \multirow{4}{*}{ F10 } & AVG & $5.4854 E+03$ & $6.6088 E+03$ & $6.9340 E+03$ & $6.5450 E+03$ & $7.8922 E+03$ & $7.4870 E+03$ & $6.7032 E+03$ \\
\hline & STD & $4.6850 E+02$ & $7.0586 E+02$ & $8.7016 E+02$ & $9.6760 E+02$ & $4.8577 E+02$ & $6.6709 E+02$ & $7.1353 E+02$ \\
\hline & Rank & 1 & 3 & 5 & 2 & 7 & 6 & 4 \\
\hline & $p$ value & & $3.5152 E-06$ & $3.8822 E-06$ & $5.7924 E-05$ & $1.7344 E-06$ & $1.7344 E-06$ & $3.5152 E-06$ \\
\hline \multirow{4}{*}{ F11 } & AVG & $1.3061 E+03$ & $2.7691 E+03$ & $3.5652 E+03$ & $2.6648 E+03$ & $2.2559 E+03$ & $4.2405 E+03$ & $4.6712 E+03$ \\
\hline & STD & $5.8914 E+01$ & $8.1162 E+02$ & $8.0204 E+02$ & $7.1733 E+02$ & $4.2647 E+02$ & $1.5082 E+03$ & $2.1153 E+03$ \\
\hline & Rank & 1 & 4 & 5 & 3 & 2 & 6 & 7 \\
\hline & $p$ value & & $1.7344 E-06$ & $1.7344 E-06$ & $1.7344 E-06$ & $1.7344 E-06$ & $1.7344 E-06$ & $1.7344 E-06$ \\
\hline \multirow{4}{*}{ F12 } & AVG & $2.4360 E+06$ & $9.8130 E+08$ & $8.2218 E+08$ & $1.3739 E+08$ & $7.9127 E+07$ & $3.2303 E+09$ & $3.1765 E+08$ \\
\hline & STD & $1.5471 E+06$ & $1.1552 E+09$ & $5.5155 E+08$ & $1.0049 E+08$ & $4.7219 E+07$ & $2.2913 E+09$ & $6.8408 E+08$ \\
\hline & Rank & 1 & 6 & 5 & 3 & 2 & 7 & 4 \\
\hline & $p$ value & & $1.7344 E-06$ & $1.7344 E-06$ & $1.7344 E-06$ & $1.7344 E-06$ & $1.7344 E-06$ & $1.7344 E-06$ \\
\hline \multirow{4}{*}{ F13 } & AVG & $2.8953 E+04$ & $9.1760 E+07$ & $1.0060 E+08$ & $7.6109 E+05$ & $1.1932 E+06$ & $4.4095 E+08$ & $2.3929 E+07$ \\
\hline & STD & $2.2290 E+04$ & $2.5341 E+08$ & $1.2133 E+08$ & $5.3474 E+05$ & $1.1894 E+06$ & $8.1558 E+08$ & $6.7495 E+07$ \\
\hline & Rank & 1 & 5 & 6 & 2 & 3 & 7 & 4 \\
\hline & $p$ value & & $1.7344 E-06$ & $1.7344 E-06$ & $1.7344 E-06$ & $1.7344 E-06$ & $1.7344 E-06$ & $1.7344 E-06$ \\
\hline \multirow{4}{*}{ F14 } & AVG & $5.6939 E+04$ & $1.4304 E+06$ & $1.2065 E+06$ & $1.8174 E+06$ & $9.9752 E+05$ & $8.4350 E+05$ & $1.6400 E+06$ \\
\hline & STD & $4.2722 E+04$ & $8.4116 E+05$ & $9.2598 E+05$ & $1.8577 E+06$ & $1.2010 E+06$ & $6.5140 E+05$ & $2.1374 E+06$ \\
\hline & Rank & 1 & 5 & 4 & 7 & 3 & 2 & 6 \\
\hline & $p$ value & & $1.7344 E-06$ & $1.9209 E-06$ & $1.7344 E-06$ & $8.4661 E-06$ & $2.6033 E-06$ & $8.4661 E-06$ \\
\hline
\end{tabular}


TABle 4: Continued.

\begin{tabular}{|c|c|c|c|c|c|c|c|c|}
\hline & & MEWOA & OBWOA & ACWOA & BWOA & BMWOA & CCMWOA & CWOA \\
\hline \multirow{4}{*}{ F15 } & AVG & $1.4040 E+04$ & $2.2774 E+06$ & $1.5036 E+07$ & $4.6385 E+05$ & $4.0735 E+05$ & $1.5924 E+07$ & $1.1770 E+07$ \\
\hline & STD & $.3322 E+04$ & $3.0346 E+06$ & $2.0563 E+07$ & $7.6165 E+05$ & $4.8751 E+05$ & $3.3323 E+07$ & $2.3834 E+07$ \\
\hline & Rank & 1 & 4 & 6 & 3 & 2 & 7 & 5 \\
\hline & $p$ value & & $1.7344 E-06$ & $1.7344 E-06$ & $1.7344 E-06$ & $1.9209 E-06$ & $1.7344 E-06$ & $1.7344 E-06$ \\
\hline \multirow{4}{*}{ F16 } & AVG & $2.8685 E+03$ & $4.1845 E+03$ & $3.9644 E+03$ & $4.1107 E+03$ & $3.6995 E+03$ & $4.2443 E+03$ & $4.0025 E+03$ \\
\hline & STD & $3.6172 E+02$ & $6.1591 E+02$ & $3.8061 E+02$ & $5.2914 E+02$ & $4.2616 E+02$ & $6.7556 E+02$ & $5.5613 E+02$ \\
\hline & Rank & 1 & 6 & 3 & 5 & 2 & 7 & 4 \\
\hline & $p$ value & & $1.9209 E-06$ & $1.7344 E-06$ & $1.7344 E-06$ & $3.8822 E-06$ & $1.7344 E-06$ & $3.5152 E-06$ \\
\hline \multirow{4}{*}{ F17 } & AVG & $2.2854 E+03$ & $2.7068 E+03$ & $2.6770 E+03$ & $2.6352 E+03$ & $2.5517 E+03$ & $2.8414 E+03$ & $2.6316 E+03$ \\
\hline & STD & $1.9620 E+02$ & $4.3177 E+02$ & $2.5201 E+02$ & $2.6185 E+02$ & $2.4464 E+02$ & $4.2162 E+02$ & $3.4749 E+02$ \\
\hline & Rank & 1 & 6 & 5 & 4 & 2 & 7 & 3 \\
\hline & $p$ value & & $1.9729 E-05$ & $2.1630 E-05$ & $3.5152 E-06$ & $2.8308 E-04$ & $5.2165 E-06$ & $1.4773 E-04$ \\
\hline \multirow{4}{*}{ F18 } & AVG & $5.5120 E+05$ & $2.8676 E+06$ & $5.4427 E+06$ & $6.4180 E+06$ & $5.3972 E+06$ & $1.2420 E+07$ & $6.6179 E+06$ \\
\hline & STD & $4.8939 E+05$ & $3.0952 E+06$ & $5.3048 E+06$ & $7.5511 E+06$ & $5.4387 E+06$ & $9.8233 E+06$ & $5.5669 E+06$ \\
\hline & Rank & 1 & 2 & 4 & 5 & 3 & 7 & 6 \\
\hline & $p$ value & & $5.3070 E-05$ & $5.7517 E-06$ & $1.9209 E-06$ & $5.2165 E-06$ & $3.1817 E-06$ & $9.3157 E-06$ \\
\hline \multirow{4}{*}{ F19 } & AVG & $1.3717 E+04$ & $3.0480 E+06$ & $1.0422 E+07$ & $4.6951 E+06$ & $2.0680 E+06$ & $1.0663 E+07$ & $1.2514 E+07$ \\
\hline & STD & $1.1887 E+04$ & $2.2545 E+06$ & $9.7587 E+06$ & $3.9463 E+06$ & $2.4791 E+06$ & $1.4257 E+07$ & $2.9116 E+07$ \\
\hline & Rank & 1 & 3 & 5 & 4 & 2 & 6 & 7 \\
\hline & $p$ value & & $1.7344 E-06$ & $1.7344 E-06$ & $1.7344 E-06$ & $1.7344 E-06$ & $1.7344 E-06$ & $1.7344 E-06$ \\
\hline \multirow{4}{*}{$\mathrm{F} 20$} & AVG & $2.5860 E+03$ & $2.7810 E+03$ & $2.7721 E+03$ & $2.7280 E+03$ & $2.7335 E+03$ & $2.7675 E+03$ & $2.8115 E+03$ \\
\hline & STD & $2.4998 E+02$ & $1.8726 E+02$ & $1.4354 E+02$ & $1.7856 E+02$ & $1.8055 E+02$ & $1.4773 E+02$ & $2.8444 E+02$ \\
\hline & Rank & 1 & 6 & 5 & 2 & 3 & 4 & 7 \\
\hline & $p$ value & & $4.6818 E-03$ & $3.1618 E-03$ & $3.6826 E-02$ & $1.8519 E-02$ & $7.7309 E-03$ & $1.5927 E-03$ \\
\hline \multirow{4}{*}{$\mathrm{F} 21$} & AVG & $2.4913 E+03$ & $2.6218 E+03$ & $2.5934 E+03$ & $2.6143 E+03$ & $2.5482 E+03$ & $2.6334 E+03$ & $2.6339 E+03$ \\
\hline & STD & $5.3196 E+01$ & $4.0524 E+01$ & $3.9408 E+01$ & $5.7246 E+01$ & $4.3122 E+01$ & $4.5956 E+01$ & $4.5301 E+01$ \\
\hline & Rank & 1 & 5 & 3 & 4 & 2 & 6 & 7 \\
\hline & $p$ value & & $2.3534 E-06$ & $2.8786 E-06$ & $6.9838 E-06$ & $4.1955 E-04$ & $2.1266 E-06$ & $3.5152 E-06$ \\
\hline \multirow{4}{*}{ F22 } & AVG & $6.7754 E+03$ & $7.4885 E+03$ & $5.7450 E+03$ & $6.0676 E+03$ & $5.7389 E+03$ & $8.0936 E+03$ & $7.6980 E+03$ \\
\hline & STD & $8.1126 E+02$ & $1.6154 E+03$ & $2.0982 E+03$ & $2.7007 E+03$ & $3.1010 E+03$ & $1.1558 E+03$ & $1.4657 E+03$ \\
\hline & Rank & 4 & 5 & 2 & 3 & 1 & 7 & 6 \\
\hline & $p$ value & & $9.8421 E-03$ & $3.3269 E-02$ & $2.4519 E-01$ & $2.4308 E-02$ & $9.7110 E-05$ & $4.8969 E-04$ \\
\hline \multirow{4}{*}{$\mathrm{F} 23$} & $\mathrm{AVG}$ & $7 E+03$ & $3.0749 E+03$ & $3.0869 E+03$ & $3.1063 E+03$ & $2.9653 E+03$ & $3.2383 E+03$ & $3.1038 E+03$ \\
\hline & STD & $5.7887 E+01$ & $9.9219 E+01$ & $1.0268 E+02$ & $9.7098 E+01$ & $8.3624 E+01$ & $1.3477 E+02$ & $8.9816 E+01$ \\
\hline & Rank & 1 & 3 & 4 & 6 & 2 & 7 & 5 \\
\hline & $p$ value & & $1.7344 E-06$ & $1.7344 E-06$ & $1.9209 E-06$ & $1.6394 E-05$ & $1.7344 E-06$ & $1.7344 E-06$ \\
\hline \multirow{4}{*}{$\mathrm{F} 24$} & AVG & $3.0070 E+03$ & $3.2234 E+03$ & $3.2273 E+03$ & $3.1919 E+03$ & $3.1389 E+03$ & $3.3596 E+03$ & $3.2316 E+03$ \\
\hline & STD & $5.7504 E+01$ & $1.0143 E+02$ & $5.8657 E+01$ & $9.1384 E+01$ & $1.0181 E+02$ & $1.1491 E+02$ & $1.0423 E+02$ \\
\hline & Rank & 1 & 4 & 5 & 3 & 2 & 7 & 6 \\
\hline & $p$ value & & $2.8786 E-06$ & $1.7344 E-06$ & $3.1817 E-06$ & $2.1630 E-05$ & $1.7344 E-06$ & $2.1266 E-06$ \\
\hline \multirow{4}{*}{ F25 } & AVG & $2.9117 E+03$ & $3.1983 E+03$ & $3.2366 E+03$ & $3.0750 E+03$ & $31 E+03$ & $3.5568 E+03$ & $3.1342 E+03$ \\
\hline & STD & $1.9595 E+01$ & $1.2767 E+02$ & $1.1942 E+02$ & $5.0411 E+01$ & $4.1369 E+01$ & $1.6227 E+02$ & $1.2156 E+02$ \\
\hline & Rank & 1 & 5 & 6 & 2 & 3 & 7 & 4 \\
\hline & $p$ value & & $1.7344 E-06$ & $1.7344 E-06$ & $1.7344 E-06$ & $1.7344 E-06$ & $1.7344 E-06$ & $1.7344 E-06$ \\
\hline \multirow{4}{*}{ F26 } & AVG & $5.8306 E+03$ & $8.1949 E+03$ & $7.7996 E+03$ & $8.2172 E+03$ & $7.2992 E+03$ & $9.0800 E+03$ & $8.1757 E+03$ \\
\hline & STD & $6.3000 E$ & $1.2501 E+03$ & $9.6542 E+02$ & $1.4063 E+03$ & $1.4449 E+03$ & $1.1041 E+03$ & $7.9669 E+02$ \\
\hline & Rank & 1 & 5 & 3 & 6 & 2 & 7 & 4 \\
\hline & $p$ value & & $5.2165 E-06$ & $2.3534 E-06$ & $7.6909 E-06$ & $4.0715 E-05$ & $1.9209 E-06$ & $1.7344 E-06$ \\
\hline \multirow{4}{*}{ F27 } & AVG & $3.4622 E+03$ & $3.4347 E+03$ & $3.5255 E+03$ & $3.4216 E+03$ & $3.3516 E+03$ & $3.6760 E+03$ & $3.4140 E+03$ \\
\hline & STD & $1.5188 E+02$ & $1.3233 E+02$ & $1.2672 E+02$ & $1.2677 E+02$ & $8.4571 E+01$ & $1.5086 E+02$ & $1.7922 E+02$ \\
\hline & Rank & 5 & 4 & 6 & 3 & 1 & 7 & 2 \\
\hline & $p$ value & & $3.7094 E-01$ & $1.3591 E-01$ & $3.7094 E-01$ & $2.2551 E-03$ & $2.3704 E-05$ & $1.4704 E-01$ \\
\hline \multirow{4}{*}{ F28 } & AVG & $8.3338 E+03$ & $3.9002 E+03$ & $3.9416 E+03$ & $3.4953 E+03$ & $3.4937 E+03$ & $4.9119 E+03$ & $3.6342 E+03$ \\
\hline & STD & $1.2992 E+03$ & $2.8345 E+02$ & $3.0133 E+02$ & $9.2327 E+01$ & $7.6587 E+01$ & $3.5365 E+02$ & $2.4510 E+02$ \\
\hline & Rank & 7 & 4 & 5 & 2 & 1 & 6 & 3 \\
\hline & $p$ value & & $1.7344 E-06$ & $1.7344 E-06$ & $1.7344 E-06$ & $1.7344 E-06$ & $1.7344 E-06$ & $1.7344 E-06$ \\
\hline & $\mathrm{AVG}$ & $4.1437 E+03$ & $4.9914 E+03$ & $4.9150 E+03$ & $5.3323 E+03$ & $4.8399 E+03$ & $5.4105 E+03$ & $4.9190 E+03$ \\
\hline & STD & $3.1912 E+02$ & $3.7880 E+02$ & $4.6601 E+02$ & $4.9795 E+02$ & $2.8342 E+02$ & $5.9734 E+02$ & $3.4227 E+02$ \\
\hline F29 & Rank & 1 & 5 & 3 & 6 & 2 & 7 & 4 \\
\hline & $p$ value & & $1.7344 E-06$ & $6.3391 E-06$ & $1.7344 E-06$ & $1.9209 E-06$ & $2.3534 E-06$ & $1.7344 E-06$ \\
\hline
\end{tabular}


TABle 4: Continued.

\begin{tabular}{ccccccccc}
\hline & & MEWOA & OBWOA & ACWOA & BWOA & BMWOA & CCMWOA & CWOA \\
\hline \multirow{4}{*}{ F30 } & AVG & $3.6123 E+04$ & $6.5293 E+07$ & $7.3992 E+07$ & $2.4908 E+07$ & $8.6846 E+06$ & $1.3551 E+08$ & $2.0455 E+07$ \\
& STD & $2.6425 E+04$ & $1.6904 E+08$ & $4.6210 E+07$ & $2.2064 E+07$ & $5.5000 E+06$ & $1.2208 E+08$ & $1.5758 E+07$ \\
& Rank & 1 & 5 & 6 & 4 & 2 & 7 & 3 \\
& $p$ value & & $1.7344 E-06$ & $1.7344 E-06$ & $1.7344 E-06$ & $1.7344 E-06$ & $1.7344 E-06$ & $1.7344 E-06$ \\
\hline
\end{tabular}

the test functions with the aim of making them more diverse. We used a total of 27 functions for testing, where F1-F8 denote Unimodal functions, F8-F13 are Multimodal functions, F14-F19 are Hybrid functions, and F20-F27 are Composition functions. The specific experimental results are shown in Table 6, where the significance of the indicators that appear is explained in the previous section. In addition, we have chosen two functions in each class as examples and depicted the convergence curves of each algorithm on the corresponding functions.

Both Unimodal and Multimodal functions, F1-F13, are taken from the benchmark functions, while F14-F27 are taken from the CEC2014 test set. The entire performance of the MEWOA can be ranked in the middle position among all algorithms in terms of Unimodal and Multimodal functions. The overall performance of MEWOA is quite good due to the presence of algorithms like HCLPSO, LSHADE, and other algorithms with extremely strong performance inside the algorithms involved in the comparison. From the convergence curves in Figure 7, we can also find that the MEWOA works very well on F1, F2, F11, and F12, in terms of both convergence speed and convergence accuracy, especially for F11, where MEWOA can reach the optimal convergence accuracy at one-third of the iterations, and this performance is also worthy of recognition.

As for the Hybrid functions and the Composition functions, MEWOA's effect is not very good, but in the two functions F21 and F23, its effect is still relatively good. We can see that on these two functions, MEWOA's ranking can be located in the middle and upper. And among some remaining functions, such as F16, F17, F19, F20, and F22, although the ranking of MEWOA is not satisfactory, the difference between them is minimal.

Owing to the strong optimization capability, the MEWOA can be applied to many other promising problems such as medical science $[125,126]$, financial risk prediction $[19,35]$, and video deblurring [127-129]. In addition, MEWOA can also be used for parameter tuning for the convolutional neural networks [127, 130-132]. Other potential applications include feature selection [133-136], parameter optimization of solar cell [137-141], social recommendation and QoS-aware service composition [142-144], brain function network decomposition and estimation [145, 146], image editing [147-149], image dehazing [130, 150-152], blockchain technology [153-155], prediction problems in the educational field [156-160], and computer vision [161-163], which are also interesting topics that are worthy of investigation in the near future.

4.7. Practical Constraint Modeling Problems. In this part, we apply the improved algorithm MEWOA in this paper to three engineering constraint problems to demonstrate the MEWOA algorithm's performance in the mathematical constraint modeling problems, including tension/compression spring, welding beam, and I-beam. The mathematical model's main target value is constructed through penalty functions [164] and automatically discards the infeasible solution through the heuristic algorithm. There is no need to carry out this solution's infeasibility and usually uses a recursive iteration way with each recursive call generating a new point before finding a feasible solution. So, the model constructed by penalty functions combined with the MEWOA algorithm is utilized to handle three mathematical modeling problems.

4.7.1. Tension-Compression String Design Problem. The tension/compression spring design aims to obtain the spring's minimum weight [165-167]. The model needs to iterate through the MEWOA algorithm to optimize the three design variables, including wire diameter $(d)$, the average coil diameter $(D)$, and the quantity of active coils $(N)$. The mathematical model formula is illustrated as follows:

Consider $\vec{x}=\left[\begin{array}{lll}x_{1} & x_{2} & x_{3}\end{array}\right]=\left[\begin{array}{lll}d & D & N\end{array}\right]$.

Objective function:

$$
f(\vec{x})_{\min }=x_{1}^{2} x_{2} x_{3}+2 x_{1}^{2} x_{2}
$$

subject to

$$
\begin{aligned}
& h_{1}(\vec{x})=1-\frac{x_{2}^{3} x_{3}}{71785 x_{1}^{4}} \leq 0, \\
& h_{2}(\vec{x})=\frac{4 x_{2}^{2}-x_{1} x_{2}}{12566\left(x_{2} x_{1}^{3}-x_{1}^{4}\right)}+\frac{1}{5180 x_{1}^{2}} \leq 0, \\
& h_{3}(\vec{x})=1-\frac{140.45 x_{1}}{x_{2}^{3} x_{3}} \leq 0, \\
& h_{4}(\vec{x})=\frac{x_{1}+x_{2}}{1.5}-1 \leq 0 .
\end{aligned}
$$

Variable ranges:

$$
\begin{aligned}
& 0.05 \leq x_{1} \leq 2.00 \\
& 0.25 \leq x_{2} \leq 1.30 \\
& 2.00 \leq x_{3} \leq 15.0 .
\end{aligned}
$$

Some scholars used mathematical techniques or metaheuristic techniques to solve the model. He and Wang [168] used PSO to handle tension/compression spring design problems. Coello Coello [169] utilized genetic algorithms to settle the problem, and the final minimum weight is 

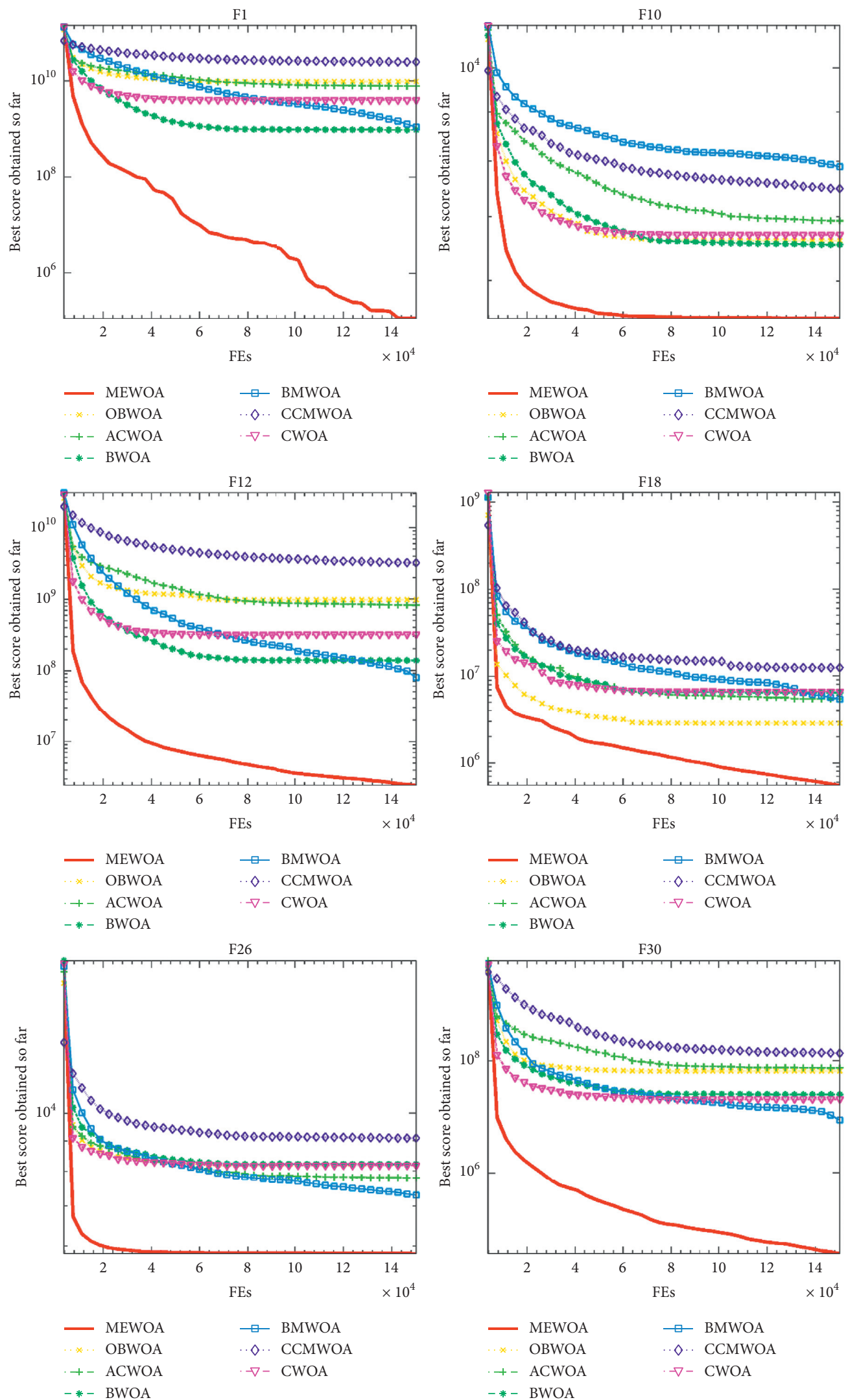

Figure 5: Convergence trends of MEWOA and peers. 
TABLE 5: Comparison results of MEWOA with metaheuristic algorithms.

\begin{tabular}{|c|c|c|c|c|c|c|c|c|}
\hline & & MEWOA & $\mathrm{HHO}$ & SMA & HGS & $\mathrm{DE}$ & MFO & CS \\
\hline \multirow{4}{*}{$\mathrm{F} 1$} & AVG & $2.7888 E+09$ & $4.9840 E+08$ & $1.8342 E+10$ & $3.1101 E+08$ & $1.5828 E+04$ & $1.4697 E+11$ & $1.0000 E+10$ \\
\hline & STD & $4.1112 E+09$ & $5.5567 E+07$ & $4.2222 E+09$ & $4.1515 E+08$ & $1.8311 E+04$ & $5.4889 E+10$ & $0.0000 E+00$ \\
\hline & Rank & 4 & 3 & 6 & 2 & 1 & 7 & 5 \\
\hline & $p$ value & & $1.5169 E-03$ & $2.3518 E-05$ & $2.5504 E-04$ & $1.8215 E-05$ & $1.8215 E-05$ & $5.6117 E-05$ \\
\hline \multirow{4}{*}{$\mathrm{F} 2$} & AVG & $1.0811 E+105$ & $4.9880 E+112$ & $1.9634 E+115$ & $2.3856 E+97$ & $1.4657 E+137$ & $6.2116 E+161$ & $1.0000 E+10$ \\
\hline & STD & $5.2928 E+105$ & $2.4436 E+113$ & $6.8005 E+115$ & $1.1687 E+98$ & $2.7004 E+137$ & $6.5535 E+04$ & $0.0000 E+00$ \\
\hline & Rank & 3 & 4 & 5 & 2 & 6 & 7 & 1 \\
\hline & $p$ value & & $1.5158 E-02$ & $2.3518 E-05$ & $8.5744 E-03$ & $1.8215 E-05$ & $1.8215 E-05$ & $1.8215 E-05$ \\
\hline \multirow{4}{*}{ F3 } & AVG & $2.0518 E+05$ & $2.2082 E+05$ & $2.0278 E+05$ & $1.2290 E+05$ & $6.1128 E+05$ & $6.7633 E+05$ & $5.1454 E+05$ \\
\hline & STD & $1.8131 E+04$ & $1.6309 E+04$ & $1.6408 E+04$ & $3.6076 E+04$ & $5.6384 E+04$ & $2.1096 E+05$ & $4.7395 E+04$ \\
\hline & Rank & 3 & 4 & 2 & 1 & 6 & 7 & 5 \\
\hline & $p$ value & & $8.5744 E-03$ & $3.9137 E-01$ & $1.8215 E-05$ & $1.8215 E-05$ & $1.8215 E-05$ & $1.8215 E-05$ \\
\hline \multirow{4}{*}{$\mathrm{F} 4$} & AVG & $1.1492 E+03$ & $1.0209 E+03$ & $1.8776 E+03$ & $7.7633 E+02$ & $6.2027 E+02$ & $3.1304 E+04$ & $6.8021 E+02$ \\
\hline & STD & $2.7710 E+02$ & $7.0247 E+01$ & $3.1601 E+02$ & $1.1704 E+02$ & $1.2220 E+01$ & $1.9668 E+04$ & $6.2934 E+01$ \\
\hline & Rank & 5 & 4 & 6 & 3 & 1 & 7 & 2 \\
\hline & $p$ value & & $5.9333 E-02$ & $1.8215 E-05$ & $1.8215 E-05$ & $1.8215 E-05$ & $1.8215 E-05$ & $1.8215 E-05$ \\
\hline \multirow{4}{*}{ F5 } & AVG & $1.5433 E+03$ & $1.5048 E+03$ & $1.3493 E+03$ & $1.2086 E+03$ & $1.4145 E+03$ & $1.8676 E+03$ & $1.3472 E+03$ \\
\hline & STD & $1.4621 E+02$ & $4.5375 E+01$ & $4.1080 E+01$ & $8.6658 E+01$ & $2.5021 E+01$ & $1.6102 E+02$ & $5.4429 E+01$ \\
\hline & Rank & 6 & 5 & 3 & 1 & 4 & 7 & 2 \\
\hline & $p$ value & & $2.5310 E-01$ & $6.3342 E-05$ & $2.6691 E-05$ & $8.2921 E-04$ & $5.6117 E-05$ & $3.0270 E-05$ \\
\hline \multirow{4}{*}{ F6 } & AVG & $6.7711 E+02$ & $6.8224 E+02$ & $6.6040 E+02$ & $6.3323 E+02$ & $6.0000 E+02$ & $6.7095 E+02$ & $6.7323 E+02$ \\
\hline & STD & $1.1545 E+01$ & $4.4768 E+00$ & $4.3507 E+00$ & $5.6014 E+00$ & $2.3705 E-13$ & $4.6354 E+00$ & $8.7010 E+00$ \\
\hline & Rank & 6 & 7 & 3 & 2 & 1 & 4 & 5 \\
\hline & $p$ value & & $2.7807 E-02$ & $3.4301 E-05$ & $1.8215 E-05$ & $1.8215 E-05$ & $3.4491 E-02$ & $1.5313 E-01$ \\
\hline \multirow{4}{*}{ F7 } & AVG & $2.6149 E+03$ & $3.7113 E+03$ & $2.8342 E+03$ & $2.0231 E+03$ & $1.7360 E+03$ & $4.8578 E+03$ & $2.3608 E+03$ \\
\hline & STD & $4.0557 E+02$ & $1.3218 E+02$ & $1.1746 E+02$ & $1.8844 E+02$ & $2.6302 E+01$ & $9.6042 E+02$ & $1.0809 E+02$ \\
\hline & Rank & 4 & 6 & 5 & 2 & 1 & 7 & 3 \\
\hline & $p$ value & & $2.0706 E-05$ & $1.1927 E-02$ & $7.1443 E-05$ & $1.8215 E-05$ & $1.8215 E-05$ & $6.0907 E-03$ \\
\hline \multirow{4}{*}{ F8 } & AVG & 03 & $1.9423 E+03$ & $1.7220 E+03$ & $1.5410 E+03$ & $1.6970 E+03$ & $2.1830 E+03$ & $1.6931 E+03$ \\
\hline & STD & $1.5719 E+02$ & $7.7800 E+01$ & $8.3696 E+01$ & $9.5254 E+01$ & $2.2908 E+01$ & $1.6552 E+02$ & $6.3349 E+01$ \\
\hline & Rank & 5 & 6 & 4 & 1 & 3 & 7 & 2 \\
\hline & $p$ value & & $7.8806 E-03$ & $1.0172 E-03$ & $3.0270 E-05$ & $4.9082 E-04$ & $2.3518 E-05$ & $2.0378 E-04$ \\
\hline \multirow{4}{*}{ F9 } & AVG & $4.0928 E+04$ & $4.8779 E+04$ & $3.3534 E+04$ & $2.4577 E+04$ & $1.4344 E+03$ & $4.4792 E+04$ & $5.1247 E+04$ \\
\hline & STD & $1.0060 E+04$ & $4.7643 E+03$ & $3.7290 E+03$ & $3.1871 E+03$ & $1.9961 E+02$ & $6.6036 E+03$ & $8.9405 E+03$ \\
\hline & Rank & 4 & 6 & 3 & 2 & 1 & 5 & 7 \\
\hline & $p$ value & & $1.6731 E-03$ & $4.2747 E-03$ & $3.0270 E-05$ & $1.8215 E-05$ & $7.1861 E-02$ & $3.9582 E-04$ \\
\hline \multirow{4}{*}{ F10 } & AVG & $1.7953 E+04$ & $2.0257 E+04$ & $1.9024 E+04$ & $1.4537 E+04$ & $2.9970 E+04$ & $1.7968 E+04$ & $1.9589 E+04$ \\
\hline & STD & $1.6953 E+03$ & $1.0935 E+03$ & $1.4198 E+03$ & $1.4226 E+03$ & $6.0852 E+02$ & $1.3604 E+03$ & $4.2207 E+02$ \\
\hline & Rank & 2 & 6 & 4 & 1 & 7 & 3 & 5 \\
\hline & $p$ value & & $1.8194 E-04$ & $4.8675 E-02$ & $4.9677 E-05$ & $1.8215 E-05$ & $9.7721 E-01$ & $3.9582 E-04$ \\
\hline \multirow{4}{*}{ F11 } & AVG & $1.1146 E+04$ & $6.6177 E+03$ & $1.3000 E+04$ & $4.0275 E+03$ & $8.0747 E+03$ & $1.6383 E+05$ & $4.2994 E+03$ \\
\hline & STD & $5.1184 E+03$ & $1.2893 E+03$ & $4.6483 E+03$ & $2.9372 E+03$ & $1.6194 E+03$ & $1.0082 E+05$ & $6.9278 E+02$ \\
\hline & Rank & 5 & 3 & 6 & 1 & 4 & 7 & 2 \\
\hline & $p$ value & & $1.5169 E-03$ & $1.0340 E-01$ & $1.0203 E-04$ & $2.3999 E-02$ & $2.0706 E-05$ & $3.4301 E-05$ \\
\hline \multirow{4}{*}{ F12 } & AVG & $3.0433 E+08$ & $5.0870 E+08$ & $1.1246 E+09$ & $1.8517 E+08$ & $2.4746 E+08$ & $3.6693 E+10$ & $1.0000 E+10$ \\
\hline & STD & $4.7772 E+08$ & $1.6876 E+08$ & $4.3599 E+08$ & $1.4342 E+08$ & $3.9693 E+07$ & $1.4776 E+10$ & $0.0000 E+00$ \\
\hline & Rank & 3 & 4 & 5 & 1 & 2 & 7 & 6 \\
\hline & $p$ value & & $1.9137 E-02$ & $5.6117 E-05$ & $7.7510 E-01$ & $1.0340 E-01$ & $1.8215 E-05$ & $1.8215 E-05$ \\
\hline \multirow{4}{*}{ F13 } & AVG & $8.0205 E+05$ & $7.6973 E+06$ & $8.3991 E+06$ & $3.7945 E+06$ & $8.9851 E+03$ & $7.4539 E+09$ & $1.0000 E+10$ \\
\hline & STD & $2.0468 E+06$ & $1.5882 E+06$ & $2.6856 E+07$ & $1.2600 E+07$ & $1.2759 E+04$ & $5.9644 E+09$ & $0.0000 E+00$ \\
\hline & Rank & 2 & 4 & 5 & 3 & 1 & 6 & 7 \\
\hline & $p$ value & & $2.0706 E-05$ & $4.9082 E-04$ & $2.3014 E-01$ & $1.8215 E-05$ & $1.8215 E-05$ & $1.8215 E-05$ \\
\hline \multirow{4}{*}{ F14 } & AVG & $1.6213 E+06$ & $2.2404 E+06$ & $2.1552 E+06$ & $8.1350 E+05$ & $1.1498 E+07$ & $1.0914 E+07$ & $3.3779 E+05$ \\
\hline & STD & $1.1996 E+06$ & $6.8718 E+05$ & $1.2730 E+06$ & $3.6761 E+05$ & $2.9606 E+06$ & $1.4342 E+07$ & $1.4734 E+05$ \\
\hline & Rank & 3 & 5 & 4 & 2 & 7 & 6 & 1 \\
\hline & $p$ value & & $1.0995 E-02$ & $9.7491 E-02$ & $4.9082 E-04$ & $1.8215 E-05$ & $3.2520 E-03$ & $1.8215 E-05$ \\
\hline & AVG & $1.0476 E+05$ & $2.0463 E+06$ & $5.4296 E+04$ & $9.2208 E+04$ & $2.6462 E+04$ & $1.7237 E+09$ & $1.0000 E+10$ \\
\hline & STD & $2.3658 E+05$ & $5.6835 E+05$ & $9.1684 E+04$ & $1.7587 E+05$ & $2.9456 E+04$ & $2.1518 E+09$ & $0.0000 E+00$ \\
\hline F15 & Rank & 4 & 5 & 2 & 3 & 1 & 6 & 7 \\
\hline & $p$ value & & $1.8215 E-05$ & $1.6151 E-01$ & $3.6057 E-01$ & $5.2963 E-01$ & $4.9677 E-05$ & $1.8215 E-05$ \\
\hline
\end{tabular}


TABle 5: Continued.

\begin{tabular}{|c|c|c|c|c|c|c|c|c|}
\hline & & MEWOA & $\mathrm{HHO}$ & SMA & HGS & $\mathrm{DE}$ & MFO & CS \\
\hline \multirow{4}{*}{ F16 } & AVG & $6.5007 E+03$ & $7.6919 E+03$ & $6.4449 E+03$ & $6.7451 E+03$ & $8.9299 E+03$ & $8.3383 E+03$ & $6.5108 E+03$ \\
\hline & STD & $6.6524 E+02$ & $5.4911 E+02$ & $9.0734 E+02$ & $7.1938 E+02$ & $3.9103 E+02$ & $6.5950 E+02$ & $2.7308 E+02$ \\
\hline & Rank & 2 & 5 & 1 & 4 & 7 & 6 & 3 \\
\hline & $p$ value & & $4.9677 E-05$ & $7.9707 E-01$ & $2.1923 E-01$ & $1.8215 E-05$ & $1.8215 E-05$ & $8.4148 E-01$ \\
\hline \multirow{4}{*}{ F17 } & AVG & $6.3650 E+03$ & $6.4839 E+03$ & $5.2020 E+03$ & $5.4345 E+03$ & $5.4888 E+03$ & $2.9574 E+04$ & $5.0701 E+03$ \\
\hline & STD & $6.0952 E+02$ & $5.4838 E+02$ & $5.7154 E+02$ & $3.8989 E+02$ & $2.3292 E+02$ & $7.7040 E+04$ & $2.3818 E+02$ \\
\hline & Rank & 5 & 6 & 2 & 3 & 4 & 7 & 1 \\
\hline & $p$ value & & $7.1032 E-01$ & $3.8838 E-05$ & $1.0203 E-04$ & $8.0517 E-05$ & $1.8215 E-05$ & $2.6691 E-05$ \\
\hline \multirow{4}{*}{ F18 } & AVG & $2.3586 E+06$ & $3.6828 E+06$ & $3.0654 E+06$ & $2.7563 E+06$ & $2.1707 E+07$ & $1.6840 E+07$ & $1.7082 E+06$ \\
\hline & STD & $1.3657 E+06$ & $1.6591 E+06$ & $1.8227 E+06$ & $1.8969 E+06$ & $6.1519 E+06$ & $2.5829 E+07$ & $4.1136 E+05$ \\
\hline & Rank & 2 & 5 & 4 & 3 & 7 & 6 & 1 \\
\hline & $p$ value & & $4.6756 E-03$ & $2.6516 E-01$ & $5.2963 E-01$ & $1.8215 E-05$ & $2.2346 E-03$ & $6.3291 E-02$ \\
\hline \multirow{4}{*}{ F19 } & AVG & $2.2811 E+04$ & $9.2444 E+06$ & $4.1479 E+05$ & $8.0893 E+04$ & $1.9794 E+04$ & $1.3273 E+09$ & $1.0000 E+10$ \\
\hline & STD & $1.4499 E+04$ & $5.1528 E+06$ & $3.7123 E+05$ & $6.2020 E+04$ & $2.2278 E+04$ & $1.4732 E+09$ & $0.0000 E+00$ \\
\hline & Rank & 2 & 5 & 4 & 3 & 1 & 6 & 7 \\
\hline & $p$ value & & $1.8215 E-05$ & $1.8215 E-05$ & $7.1443 E-05$ & $6.3291 E-02$ & $1.8215 E-05$ & $1.8215 E-05$ \\
\hline \multirow{4}{*}{$\mathrm{F} 20$} & AVG & $6.2485 E+03$ & $5.7908 E+03$ & $5.0367 E+03$ & $5.4356 E+03$ & $5.7274 E+03$ & $5.9532 E+03$ & $5.6192 E+03$ \\
\hline & STD & $6.1430 E+02$ & $4.9167 E+02$ & $4.5369 E+02$ & $5.5928 E+02$ & $2.2179 E+02$ & $6.5073 E+02$ & $2.4746 E+02$ \\
\hline & Rank & 7 & 5 & 1 & 2 & 4 & 6 & 3 \\
\hline & $p$ value & & $1.5158 E-02$ & $2.3518 E-05$ & $3.1822 E-04$ & $2.9642 E-03$ & $8.6476 E-02$ & $5.4594 E-04$ \\
\hline \multirow{4}{*}{ F21 } & AVG & $3.4080 E+03$ & $4.0621 E+03$ & $3.1835 E+03$ & $2.9952 E+03$ & $3.2438 E+03$ & $3.7236 E+03$ & $3.1724 E+03$ \\
\hline & STD & $1.9244 E+02$ & $1.6810 E+02$ & $1.1195 E+02$ & $8.2857 E+01$ & $3.0492 E+01$ & $2.1205 E+02$ & $9.9310 E+01$ \\
\hline & Rank & 5 & 7 & 3 & 1 & 4 & 6 & 2 \\
\hline & $p$ value & & $1.8215 E-05$ & $6.0677 E-04$ & $1.8215 E-05$ & $1.5169 E-03$ & $2.0706 E-05$ & $9.0672 E-05$ \\
\hline \multirow{4}{*}{ F22 } & AVG & $1.9262 E+04$ & $2.4028 E+04$ & $2.1385 E+04$ & $1.7506 E+04$ & $3.1763 E+04$ & $2.0519 E+04$ & $2.2755 E+04$ \\
\hline & STD & $1.5257 E+03$ & $1.3118 E+03$ & $1.1967 E+03$ & $1.0533 E+03$ & $6.0097 E+02$ & $1.8378 E+03$ & $5.9742 E+02$ \\
\hline & Rank & 2 & 6 & 4 & 1 & 7 & 3 & 5 \\
\hline & $p$ value & & $1.8215 E-05$ & $1.1472 E-04$ & $1.4470 E-04$ & $1.8215 E-05$ & $8.5744 E-03$ & $1.8215 E-05$ \\
\hline \multirow{4}{*}{$\mathrm{F} 23$} & AVG & $3.9003 E+03$ & $5.1643 E+03$ & $3.6747 E+03$ & $3.2529 E+03$ & $3.5529 E+03$ & $3.9352 E+03$ & $3.6545 E+03$ \\
\hline & STD & $1.6807 E+02$ & $2.7838 E+02$ & $7.4514 E+01$ & $5.5872 E+01$ & $2.1217 E+01$ & $1.5414 E+02$ & $8.7300 E+01$ \\
\hline & Rank & 5 & 7 & 4 & 1 & 2 & 6 & 3 \\
\hline & $p$ value & & $1.8215 E-05$ & $5.6117 E-05$ & $1.8215 E-05$ & $1.8215 E-05$ & $3.6057 E-01$ & $2.6691 E-05$ \\
\hline \multirow{4}{*}{$\mathrm{F} 24$} & AVG & $4.6479 E+03$ & $6.5004 E+03$ & $4.3113 E+03$ & $3.9218 E+03$ & $4.1047 E+03$ & $4.4701 E+03$ & $4.2583 E+03$ \\
\hline & STD & $2.1942 E+02$ & $3.8952 E+02$ & $1.0737 E+02$ & $9.5224 E+01$ & $2.4785 E+01$ & $1.7258 E+02$ & $9.9907 E+01$ \\
\hline & Rank & 6 & 7 & 4 & 1 & 2 & 5 & 3 \\
\hline & $p$ value & & $1.8215 E-05$ & $4.3941 E-05$ & $1.8215 E-05$ & $1.8215 E-05$ & $5.5811 E-03$ & $2.0706 E-05$ \\
\hline \multirow{4}{*}{ F25 } & AVG & $3.9507 E+03$ & $3.7214 E+03$ & $4.2569 E+03$ & $3.6542 E+03$ & $3.2693 E+03$ & $1.2101 E+04$ & $3.3212 E+03$ \\
\hline & STD & $2.8356 E+02$ & $6.7331 E+01$ & $1.7083 E+02$ & $3.7974 E+02$ & $5.7511 E+01$ & $5.2221 E+03$ & $4.3663 E+01$ \\
\hline & Rank & 5 & 4 & 6 & 3 & 1 & 7 & 2 \\
\hline & $p$ value & & $2.8499 E-04$ & $6.7386 E-04$ & $1.7719 E-02$ & $1.8215 E-05$ & $1.8215 E-05$ & $1.8215 E-05$ \\
\hline \multirow{4}{*}{ F26 } & AVG & $304 E+04$ & $2.6052 E+04$ & $1.8102 E+04$ & $1.2630 E+04$ & $1.4720 E+04$ & $1.9820 E+04$ & $1.6418 E+04$ \\
\hline & STD & $2.2676 E+03$ & $1.6397 E+03$ & $3.2370 E+03$ & $2.6531 E+03$ & $2.3288 E+02$ & $1.5918 E+03$ & $1.1740 E+03$ \\
\hline & Rank & 5 & 7 & 4 & 1 & 2 & 6 & 3 \\
\hline & $p$ value & & $1.8215 E-05$ & $6.6824 E-01$ & $1.8215 E-05$ & $1.8215 E-05$ & $1.3735 E-01$ & $3.9582 E-04$ \\
\hline \multirow{4}{*}{$\mathrm{F} 27$} & AVG & $8.3736 E+03$ & $4.4735 E+03$ & $3.6246 E+03$ & $3.5247 E+03$ & $3.5497 E+03$ & $4.0464 E+03$ & $3.7779 E+03$ \\
\hline & STD & $1.7236 E+03$ & $3.2426 E+02$ & $8.3281 E+01$ & $7.9600 E+01$ & $6.2216 E+01$ & $2.3018 E+02$ & $8.0163 E+01$ \\
\hline & Rank & 7 & 6 & 3 & 1 & 2 & 5 & 4 \\
\hline & $p$ value & & $2.0706 E-05$ & $1.8215 E-05$ & $1.8215 E-05$ & $1.8215 E-05$ & $1.8215 E-05$ & $1.8215 E-05$ \\
\hline \multirow{4}{*}{ F28 } & AVG & $5.4475 E+04$ & $3.7262 E+03$ & $4.1512 E+03$ & $3.4833 E+03$ & $3.3953 E+03$ & $2.0538 E+04$ & $3.4414 E+03$ \\
\hline & STD & $5.9341 E+03$ & $4.8190 E+01$ & $2.4287 E+02$ & $4.2503 E+01$ & $1.8255 E+02$ & $1.8753 E+03$ & $3.1260 E+01$ \\
\hline & Rank & 7 & 4 & 5 & 3 & 1 & 6 & 2 \\
\hline & $p$ value & & $1.8215 E-05$ & $1.8215 E-05$ & $1.8215 E-05$ & $1.8215 E-05$ & $1.8215 E-05$ & $1.8215 E-05$ \\
\hline \multirow{4}{*}{ F29 } & AVG & $8.3827 E+03$ & $9.8509 E+03$ & $8.6041 E+03$ & $6.9585 E+03$ & $7.4441 E+03$ & $1.7671 E+04$ & $7.7750 E+03$ \\
\hline & STD & $7.1162 E+02$ & $8.7940 E+02$ & $6.7211 E+02$ & $4.3224 E+02$ & $3.0162 E+02$ & $1.5175 E+04$ & $2.5110 E+02$ \\
\hline & Rank & 4 & 6 & 5 & 1 & 2 & 7 & 3 \\
\hline & $p$ value & & $6.3342 E-05$ & $1.7932 E-01$ & $2.6691 E-05$ & $2.8499 E-04$ & $2.0706 E-05$ & $1.3743 E-03$ \\
\hline & AVG & $2.4181 E+06$ & $4.7921 E+07$ & $1.6219 E+07$ & $1.5200 E+06$ & $2.2138 E+04$ & $3.6915 E+09$ & $1.0000 E+10$ \\
\hline & STD & $2.3647 E+06$ & $1.6952 E+07$ & $1.3407 E+07$ & $2.2183 E+06$ & $7.6087 E+03$ & $2.8610 E+09$ & $0.0000 E+00$ \\
\hline F30 & Rank & 3 & 5 & 4 & 2 & 1 & 6 & 7 \\
\hline & $p$ value & & $1.8215 E-05$ & $2.6691 E-05$ & $1.5313 E-01$ & $1.8215 E-05$ & $1.8215 E-05$ & $1.8215 E-05$ \\
\hline
\end{tabular}



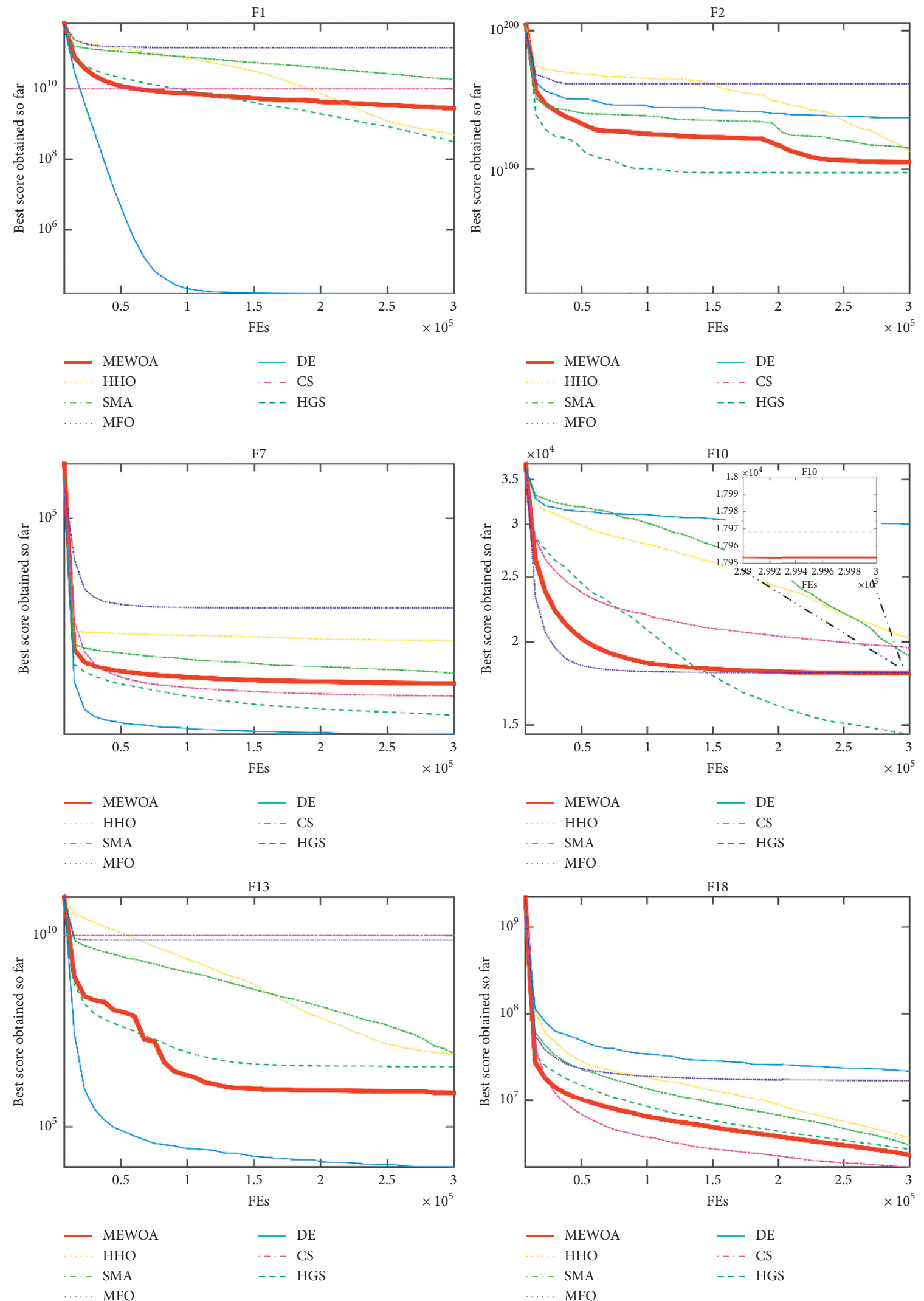

(a)

Figure 6: Continued. 

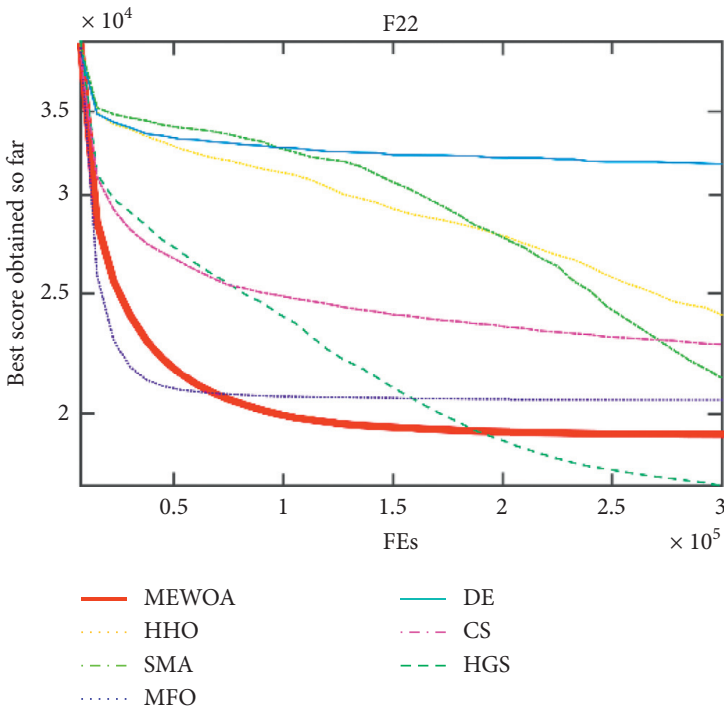
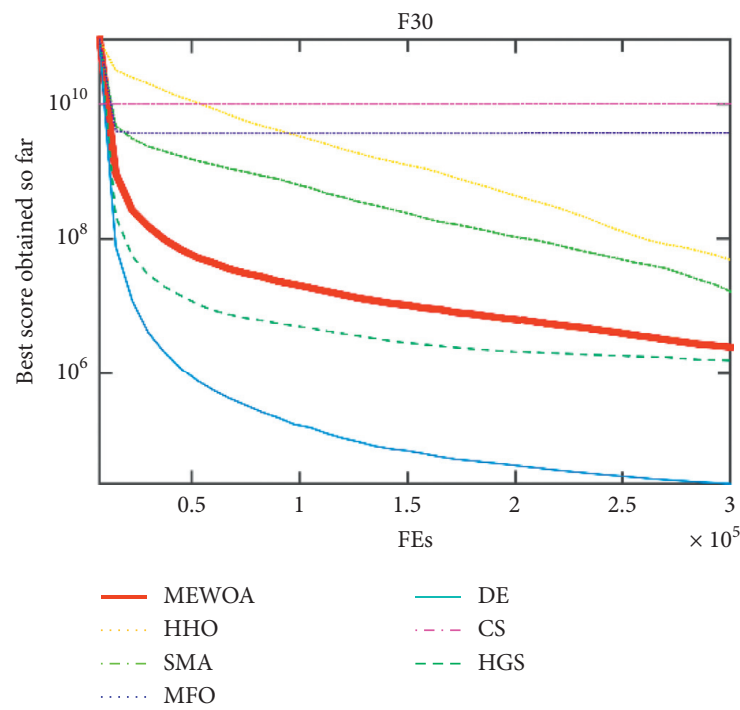

MFO

(b)

Figure 6: Convergence trends of MEWOA and metaheuristic algorithms.

TABle 6: Comparison results of MEWOA with advanced algorithms.

\begin{tabular}{|c|c|c|c|c|c|c|c|c|c|}
\hline & & MEWOA & HCLPSO & SADE & JADE & CLPSO & LSHADE & LSHADE_ES & MSCA \\
\hline \multirow{4}{*}{$\mathrm{F} 1$} & AVG & $699 E_{-}$ & 0 & $2584 F_{-}$ & $3 E-49$ & -08 & & & $5 F_{-}$ \\
\hline & & & & & & & & & \\
\hline & Rank & 3 & 1 & 7 & 4 & 8 & J & 6 & 2 \\
\hline & $p$ value & & $1.8215 E-05$ & $1.8215 E-05$ & $1.8215 E-05$ & $1.8215 E-05$ & 1.8215 & $215 E-05$ & 05 \\
\hline \multirow{4}{*}{ F2 } & AVG & & 0 & 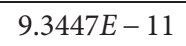 & $157 F$ & 6 & 1.5 & & 94 \\
\hline & STD & & & & $7 E$ & & & & \\
\hline & Rank & 3 & 1 & 5 & 4 & 7 & 6 & 8 & 2 \\
\hline & $p$ value & & $1.8215 \mathrm{H}$ & $1.8215 E-05$ & $1.8215 E-05$ & $1.8215 E$ & $1.8215 E$ & -05 & $1.8215 E$ \\
\hline \multirow{4}{*}{ F3 } & AVG & 3 & 00 & 02010 & $.0487 E-01$ & $220 E+04$ & 01 & & 36 \\
\hline & & & 0 & 2 & $12 E$ & 2 & & & 35 \\
\hline & Rank & 7 & 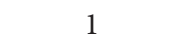 & 6 & 3 & 8 & 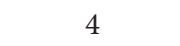 & 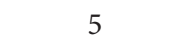 & 2 \\
\hline & $p$ value & & 05 & 04 & $1.8215 E-05$ & 05 & 1.8 & 05 & -05 \\
\hline \multirow{4}{*}{$\mathrm{F} 4$} & & & & & $2 \Gamma$ & & & & \\
\hline & & & & & 0 & & & & \\
\hline & Rank & 8 & 1 & 3 & 6 & 5 & 7 & 4 & 2 \\
\hline & $p$ value & & 1.80 & 1. & $20 E$ & 3 & & & -05 \\
\hline \multirow{4}{*}{ F5 } & & & & & & & & & \\
\hline & & 2.27 & 0 & & $26 E$ & 1 & 1 & 01 & 01 \\
\hline & Rank & 3 & 1 & 8 & 2 & 6 & 5 & 7 & 4 \\
\hline & $p$ value & & & ת & $1.2889 E$ & $42 E$ & 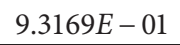 & & -05 \\
\hline \multirow{4}{*}{ F6 } & & & & & $5 E-$ & & & & \\
\hline & & 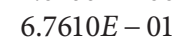 & 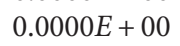 & & $53 E$ & 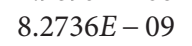 & & & \\
\hline & Rank & 7 & 1 & 5 & 2 & 6 & 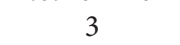 & 1 & 8 \\
\hline & $p$ value & & & 05 & $1.8215 E-05$ & 05 & $1.8215 E$ & $15 E-05$ & $.8215 E-05$ \\
\hline \multirow{4}{*}{ F7 } & & 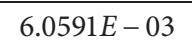 & , & 4 & 02 & 2 & 1 & & $E-04$ \\
\hline & & $014 E$ & $00 E+0$ & $25 E$ & $3.4827 E-02$ & $55 E-$ & $65 E$ & -01 & -04 \\
\hline & Rank & 3 & 1 & 6 & 5 & 4 & 8 & 7 & 2 \\
\hline & $p$ value & & & & $15 E-$ & $15 E$ & 1.8 & $15 E-05$ & $15 E-05$ \\
\hline \multirow{4}{*}{ F8 } & & -1.8 & & & -4 & -4 & -6.1 & +04 & +04 \\
\hline & STD & $2.1949 E$ & $0.0000 E+00$ & $3.2508 E+02$ & $3.7885 E+02$ & $6.9669 E$ & $4.2287 E$ & 02 & $2.6514 E$ \\
\hline & Rank & 6 & 8 & 2 & 3 & 1 & 7 & 4 & 5 \\
\hline & $p$ value & & $1.8215 E-05$ & $1.8215 E-05$ & $1.8215 E-05$ & $1.8215 E-05$ & $1.8215 E-05$ & $1.8215 E-05$ & $7.1861 E-02$ \\
\hline
\end{tabular}


TABle 6: Continued.

\begin{tabular}{|c|c|c|c|c|c|c|c|c|c|}
\hline & & MEWOA & HCLPSO & SADE & JADE & CLPSO & LSHADE & LSHADE_ES & MSCA \\
\hline \multirow{4}{*}{ F9 } & AVG & $8 E+02$ & +00 & $E+01$ & $E-01$ & $9 E-02$ & $9 E+03$ & $193 E+00$ & $0 E+00$ \\
\hline & STD & & 00 & $3751 E+00$ & & & & & \\
\hline & Rank & 7 & 1 & 6 & 4 & 3 & 8 & 5 & 1 \\
\hline & $p$ value & & $1.8215 E-05$ & $1.8215 E-05$ & $.8215 E-05$ & $1.8215 E-05$ & $6.0677 E-04$ & $1.8215 E-05$ & $.8215 E-05$ \\
\hline \multirow{4}{*}{ F10 } & $\mathrm{AVG}$ & $77 E+01$ & $0000 E+00$ & $4.8107 E+00$ & $3569 E+00$ & $6114 E-05$ & $1.8711 E-13$ & $.0971 E+01$ & $0 E+00$ \\
\hline & STD & $6291 E+00$ & $0000 E+00$ & $7.7991 E-01$ & $0222 E-01$ & & $E-14$ & & +00 \\
\hline & Rank & 8 & 1 & 5 & 4 & 3 & 2 & 7 & 6 \\
\hline & $p$ value & & 1.8215 & $2.6691 E-05$ & $2.6691 E-05$ & $2.6691 E-05$ & $2.6691 E-05$ & $520 E-03$ & $756 E-03$ \\
\hline \multirow{4}{*}{ F11 } & AVG & 0 & 0 & 1 & $Z F_{-}$ & $E-08$ & 02 & & +00 \\
\hline & STD & & .0000 & L & L & 08 & 9.00210 & & $00 E+00$ \\
\hline & Rank & 1 & 1 & 8 & 6 & 4 & 7 & 5 & 1 \\
\hline & $p$ value & & $1.0000 E+00$ & $1.3101 E-04$ & $1.8215 E-05$ & $15 E-05$ & $1.8215 E-05$ & $15 E-05$ & $1.0000 E+00$ \\
\hline \multirow{4}{*}{ F12 } & AVG & 2 & $0000 E+00$ & & & & +00 & +00 & $8 E-01$ \\
\hline & & & & & & & & & \\
\hline & Rank & 4 & 1 & 3 & 6 & 2 & - & 8 & \\
\hline & $p$ value & & $1.8215 E-05$ & $6.7464 E-02$ & $.0960 E-01$ & $215 E-05$ & $2.2346 E-03$ & $306 E-03$ & $E-01$ \\
\hline \multirow{4}{*}{ F13 } & & & & & & & & & \\
\hline & & & & & & & & & -01 \\
\hline & Rank & 5 & 1 & 4 & 3 & 2 & 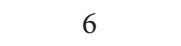 & 8 & 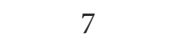 \\
\hline & $p$ value & & $1.8215 \mathrm{~L}$ & $7.4779 E$ & 05 & 05 & $3.1731 E$ & 05 & -05 \\
\hline \multirow{4}{*}{ F14 } & AVG & $6336 E+06$ & $D E+00$ & 5,06 & $62 F+05$ & $5 F_{07}$ & & & $E+07$ \\
\hline & STD & 6 & $0.0000 E+00$ & 4.4 & . & 06 & $1.295 / L$ & & $24 E+07$ \\
\hline & Rank & 6 & 1 & 5 & 2 & 7 & 3 & 4 & 8 \\
\hline & $p$ value & & $1.8215 E-05$ & $1.8215 E-05$ & $215 E-05$ & $15 E-05$ & $1.8215 E-05$ & $15 E-05$ & $15 E-05$ \\
\hline \multirow{4}{*}{ F15 } & & & & & & & & & \\
\hline & & & & & & & & & \\
\hline & Rank & 7 & 1 & 2 & 4 & 5 & 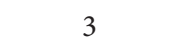 & & \\
\hline & $p$ value & & 1 & & & & 4 & & -05 \\
\hline \multirow{4}{*}{ F16 } & & & & & & & & & \\
\hline & & & & & & & & & \\
\hline & Rank & 7 & 1 & 2 & 3 & 5 & 4 & 6 & 8 \\
\hline & $p$ value & & $1.8215 E-05$ & $2.6691 E-05$ & $215 E-05$ & $18 E-05$ & $2.0706 E$ & $998 E-03$ & $5 E-05$ \\
\hline \multirow{4}{*}{ F17 } & $4 \pi$ & $11=0$ & & & & & & & \\
\hline & STD & t & 0 & & ( & 3 & & & \\
\hline & & 7 & ( & 5 & ( & 6 & ( & 2 & 8 \\
\hline & $p$ value & & $15 E-05$ & 03 & 05 & $06 E-03$ & 05 & $15 E-05$ & $42 E-05$ \\
\hline \multirow{4}{*}{ F18 } & & & & & & & & & $E+07$ \\
\hline & & & & & & & & & \\
\hline & Rank & 6 & 1 & 5 & 3 & 7 & 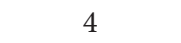 & 0 & 0 \\
\hline & $p$ value & & $5 E-05$ & & & & & & -05 \\
\hline \multirow{4}{*}{ F19 } & & & & & & & & & \\
\hline & STD & & & & & & & 02 & 02 \\
\hline & Rank & 8 & 1 & 4 & 6 & 3 & 5 & 2 & 7 \\
\hline & $p$ value & & $15 E-05$ & $91 E-05$ & $15 E-05$ & $66 E-05$ & $91 E-05$ & $15 E-05$ & $E-03$ \\
\hline \multirow{4}{*}{ F20 } & & & & & & & & & \\
\hline & STD & H & 0 & $2 D$ & $36 E$ & $86 E$ & 1 & 03 & 02 \\
\hline & Rank & 7 & 1 & 5 & 2 & 6 & - & 4 & 8 \\
\hline & $p$ value & & $5 I$ & $5 D$ & 5 & 5 & 5 & 55 & -05 \\
\hline \multirow{4}{*}{ F21 } & & & & & & & & & \\
\hline & STD & $4 E$ & $00 E+00$ & $9.4205 E+00$ & $9.7299 E+0$ & $93 E+00$ & $1.2508 E+01$ & $9061 E+01$ & -04 \\
\hline & Rank & 4 & 1 & 5 & 6 & 3 & 7 & 8 & 2 \\
\hline & $p$ value & & $1.8215 E-05$ & $3.8838 E-05$ & $1.8215 E-05$ & $677 E-05$ & $1.8215 E-05$ & $1.8215 E-05$ & $1.8215 E-05$ \\
\hline \multirow{4}{*}{ F22 } & & & 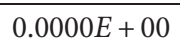 & & 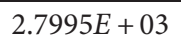 & & 2 & 03 & \\
\hline & STD & $91 \mathrm{E}$ & $0.0000 E+00$ & $1.7604 E$ & $15 E$ & $29 E$ & $57 E$ & 01 & -13 \\
\hline & Rank & 8 & 1 & 3 & 5 & 4 & 7 & 6 & 2 \\
\hline & $p$ value & & $15 E-05$ & $215 E-05$ & $577 E-04$ & $691 E-05$ & $1.5313 E-01$ & $3.9672 E-02$ & $1.8215 E-05$ \\
\hline & & & & & & & & & $47 E+03$ \\
\hline & & . & $0.0000 E$ & $7.7142 E$ & $3.5414 t$ & $3.1306 L$ & $3.3575 L$ & -02 & +02 \\
\hline $\mathrm{F} 2$ & Rank & 2 & 1 & 7 & 4 & 8 & 5 & 6 & 3 \\
\hline & $p$ value & & $1.8215 E-05$ & $3.8838 E-05$ & $3.8838 E-05$ & $3.0270 E-05$ & $3.8838 E-05$ & $3.8838 E-05$ & $1.0172 E-03$ \\
\hline
\end{tabular}


TABLE 6: Continued.

\begin{tabular}{|c|c|c|c|c|c|c|c|c|c|}
\hline & & MEWOA & HCLPSO & SADE & JADE & CLPSO & LSHADE & LSHADE_ES & MSCA \\
\hline \multirow{4}{*}{ F24 } & AVG & $6.2407 E+03$ & $0.0000 E+00$ & $4.9086 E+03$ & $4.6702 E+03$ & $5.2973 E+03$ & $5.5069 E+03$ & $5.6285 E+03$ & $6.0305 E+03$ \\
\hline & STD & $2.0232 E+02$ & $0.0000 E+00$ & $1.6887 E+02$ & $2.0066 E+02$ & $8.6157 E+01$ & $1.9325 E+02$ & $1.3858 E+02$ & $1.5676 E+02$ \\
\hline & Rank & 8 & 1 & 3 & 2 & 4 & 5 & 6 & 7 \\
\hline & $p$ value & & $1.8215 E-05$ & $1.8215 E-05$ & $1.8215 E-05$ & $1.8215 E-05$ & $1.8215 E-05$ & $1.8215 E-05$ & $2.6998 E-03$ \\
\hline \multirow{4}{*}{ F25 } & AVG & $1.1362 E+04$ & $0.0000 E+00$ & $6.7031 E+03$ & $6.3849 E+03$ & $7.6197 E+03$ & $7.8583 E+03$ & $1.0152 E+04$ & $1.4004 E+04$ \\
\hline & STD & $1.1444 E+03$ & $0.0000 E+00$ & $5.4002 E+02$ & $6.6193 E+02$ & $1.1661 E+03$ & $1.0245 E+03$ & $9.7023 E+02$ & $1.2833 E+03$ \\
\hline & Rank & 7 & 1 & 3 & 2 & 4 & 5 & 6 & 8 \\
\hline & $p$ value & & $1.8215 E-05$ & $1.8215 E-05$ & $1.8215 E-05$ & $1.8215 E-05$ & $1.8215 E-05$ & $1.6731 E-03$ & $3.4301 E-05$ \\
\hline \multirow{4}{*}{ F26 } & AVG & $3.8176 E+08$ & $0.0000 E+00$ & $6.2856 E+03$ & $4.4561 E+03$ & $1.5026 E+04$ & $4.5562 E+03$ & $5.6922 E+06$ & $4.6298 E+08$ \\
\hline & STD & $2.1098 E+08$ & $0.0000 E+00$ & $8.1826 E+02$ & $2.2726 E+02$ & $3.6346 E$ & $1.6183 E+02$ & $2.7859 E+07$ & $2.9315 E+08$ \\
\hline & Rank & 7 & 1 & 4 & 2 & 5 & 3 & 6 & 8 \\
\hline & $p$ value & & $1.8215 E-05$ & $1.8215 E-05$ & $1.8215 E-05$ & $1.8215 E-05$ & $1.8215 E-05$ & $1.8215 E-05$ & $4.5757 E-01$ \\
\hline \multirow{4}{*}{ F27 } & AVG & $1.2546 E+05$ & $0.0000 E+00$ & $3.4105 E+04$ & $1.3837 E+04$ & $6.8084 E+04$ & $1.6085 E+04$ & $1.5161 E+04$ & $7.9458 E+06$ \\
\hline & STD & $1.6955 E+05$ & $0.0000 E+00$ & $1.0053 E+04$ & $2.2441 E+03$ & $1.4559 E+04$ & $5.3295 E+03$ & $2.0803 E+03$ & $2.9451 E+06$ \\
\hline & Rank & 7 & 1 & 5 & 2 & 6 & 4 & 3 & 8 \\
\hline & $p$ value & & $1.8215 E-05$ & $3.2520 E-03$ & $1.8215 E-05$ & $1.0000 E+00$ & $1.8215 E-05$ & $1.8215 E-05$ & $1.8215 E-05$ \\
\hline
\end{tabular}

0.0127048. Some more algorithms were used to solve this problem that can be seen in the harmony search (IHS) algorithm [170] and RO algorithm [171]. Experimental results show that the weight of the model obtained by MEWOA is 0.0126788 , as shown in Table 7 , which is smaller than the minimum value obtained by other methods.

4.7.2. Welded Beam Design Problem. The aim of the welded beam design model [169] is to achieve the lowest welded beam manufacturing cost. The model includes the following four constraint variables: critical buckling load $\left(P_{c}\right)$, shear stress $(\tau)$, internal bending stress of the beam $(\theta)$, and deflection rate $(\delta)$. The steel bar height $(t)$, weld seam thickness $(h)$, steel bar thickness $(b)$, and steel bar length $(l)$ are the direct parameters that affect the manufacturing cost of welded beams. The mathematical model is as follows:

Consider $\vec{x}=\left[\begin{array}{llll}x_{1} & x_{2} & x_{3} & x_{4}\end{array}\right]=\left[\begin{array}{llll}h & l & t & b\end{array}\right]$

Objective

$$
\begin{aligned}
& f(\vec{x})_{\min }=1.10471 x_{2} x_{1}^{2}+0.04811 x_{3} x_{4}\left(14.0+x_{2}\right) \\
& \quad \text { subject to }
\end{aligned}
$$

$$
\begin{aligned}
& g_{1}(\vec{x})=\tau(\vec{x})-\tau_{\max } \leq 0, \\
& g_{2}(\vec{x})=\sigma(\vec{x})-\sigma_{\max } \leq 0, \\
& g_{3}(\vec{x})=\delta(\vec{x})-\delta_{\max } \leq 0, \\
& g_{4}(\vec{x})=x_{1}-x_{4} \leq 0, \\
& g_{5}(\vec{x})=P-P_{C}(\vec{x}) \leq 0, \\
& g_{6}(\vec{x})=0.125-x_{1} \leq 0, \\
& g_{7}(\vec{x})=1.10471 x_{1}^{2}+0.04811 x_{3} x_{4}\left(14.0+x_{2}\right)-5.0 \leq 0 .
\end{aligned}
$$

Variable ranges:

$$
\begin{aligned}
& 0.1 \leq x_{1} \leq 2 \\
& 0.1 \leq x_{2} \leq 10 \\
& 0.1 \leq x_{3} \leq 10 \\
& 0.1 \leq x_{4} \leq 2
\end{aligned}
$$

where

$$
\begin{aligned}
& (\vec{x})=\sqrt{\left(\tau^{\prime}\right)^{2}+2 \tau^{\prime} \tau^{\prime \prime} \frac{x_{2}}{2 R}+\left(\tau^{\prime \prime}\right)^{2}}, \\
& \tau^{\prime}=\frac{P}{\sqrt{2} x_{1} x_{2}}, \\
& \tau^{\prime \prime}=\frac{M R}{J}, \\
& M=P\left(L+\frac{x_{2}}{2}\right) \\
& R=\sqrt{\frac{x_{2}^{2}}{4}+\left(\frac{x_{1}+x_{3}}{2}\right)^{2}}, \\
& J=2\left\{\sqrt{2} x_{1} x_{2}\left[\frac{x_{2}^{2}}{4}+\left(\frac{x_{1}+x_{3}}{2}\right)^{2}\right]\right\} \text {, } \\
& \sigma(\vec{x})=\frac{6 P L}{x_{4} x_{3}^{2}} \\
& \delta(\vec{x})=\frac{6 P L^{3}}{E x_{3}^{2} x_{4}} \\
& P_{C}(\vec{x})=\frac{4.013 E \sqrt{\left(x_{3}^{2} x_{4}^{6} / 36\right)}}{L^{2}}\left(1-\frac{x_{3}}{2 L} \sqrt{\frac{E}{4 G}}\right) \text {, } \\
& P=60001 b, L=14 \text { in.. } \delta_{\max }=0.25 \text { in.., } \\
& E=30 \times 11^{6} \text { psi, } G=12 \times 10^{6} \text { psi, } \\
& \tau_{\max }=13600 \text { psi, } \sigma_{\max }=30000 \text { psi. }
\end{aligned}
$$



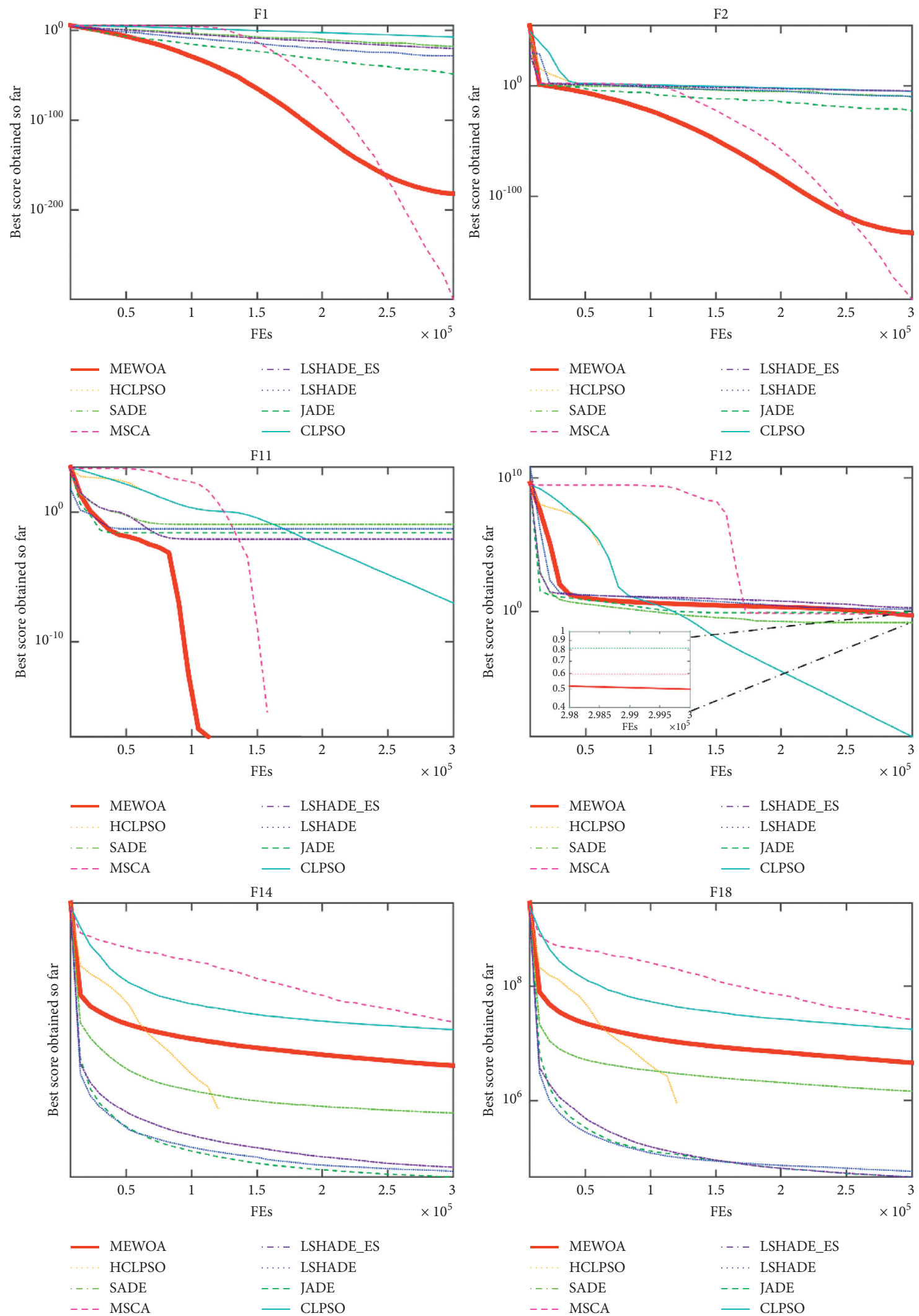

(a)

Figure 7: Continued. 


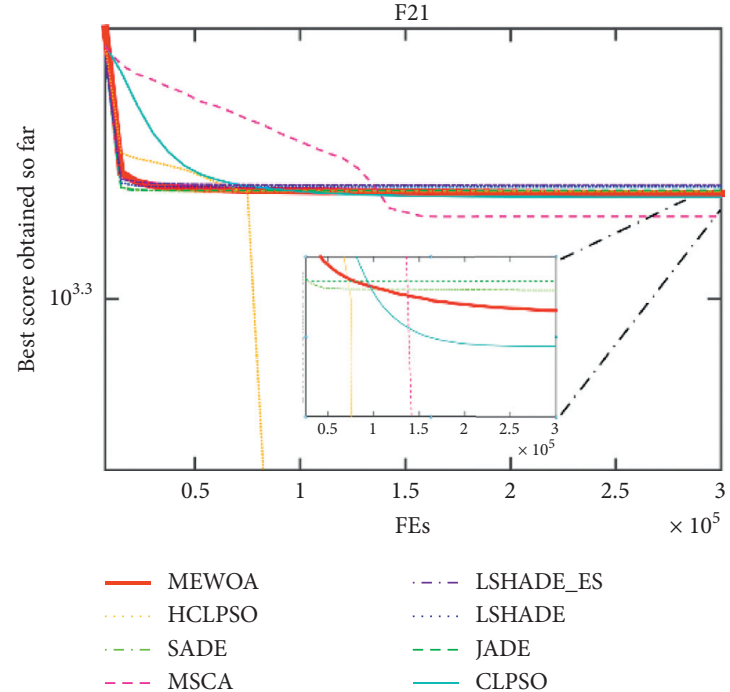

(b)

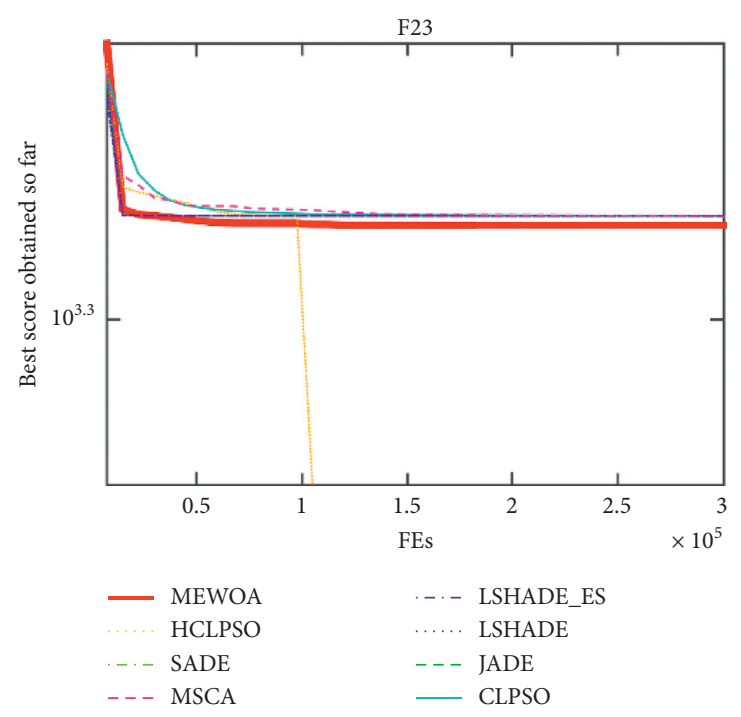

FIgURE 7: Convergence trends of MEWOA and advanced algorithms.

TABLE 7: Comparison results of MEWOA and other algorithms on the tension/compression spring case.

\begin{tabular}{|c|c|c|c|c|}
\hline \multirow{2}{*}{ Method } & \multicolumn{3}{|c|}{ Best variables } & \multirow{2}{*}{ Best weight } \\
\hline & $d$ & $N$ & $D$ & \\
\hline MEWOA & 0.051579 & 0.354086 & 11.44504 & 0.012666 \\
\hline WOA [85] & 0.051207 & 12.0043032 & 0.345215 & 0.0126763 \\
\hline PSO [168] & 0.015728 & 11.244543 & 0.357644 & 0.0126747 \\
\hline GA [169] & 0.051480 & 11.632201 & 0.351661 & 0.0127048 \\
\hline $\mathrm{RO}[171]$ & 0.051370 & 11.762790 & 0.349096 & 0.0126788 \\
\hline IHS [170] & 0.051154 & 12.076432 & 0.349871 & 0.0126706 \\
\hline Constraint correction [166] & 0.050000 & 14.250000 & 0.315900 & 0.0128334 \\
\hline Math. Optimization [167] & 0.053396 & 901854000 & 0.399180 & 0.0127303 \\
\hline
\end{tabular}

Some scholars use mathematical techniques or metaheuristic techniques to solve the model. Kaveh and Khayatazad [171] adopted RO to solve the manufacturing cost of the model. The enhanced HS model IHS [170] was also used to calculate the model's manufacturing cost. Radgsdell and Phillips [172] used Davidon-Fletcher-Powell, Richardson's random method, and the Simplex method to solve the model's minimum manufacturing cost. As shown in Table 8 , when the parameters were set to $0.1885,3.471$, 9.11343, and 0.206754, MEWOA obtained the minimum manufacturing cost of the welded beam of 1.720001 . It proves that the MEWOA possesses a very good effect in this engineering problem.

4.7.3. I-Beam Design Problem. We used the MEWOA method to solve the I-beam design problem by optimizing the four parameters, including the I-beam length, two thicknesses, and height, to get the minimum vertical deflection. The mathematical model is as follows:

$$
\text { Consider } \vec{x}=\left[\begin{array}{llll}
x_{1} & x_{2} & x_{3} & x_{4}
\end{array}\right]=\left[\begin{array}{llll}
b & h & t_{w} & t_{f}
\end{array}\right]
$$

Objective $f(\vec{x})_{\min }=5000 /\left(\left(t_{w}\left(h-2 t_{f}\right)^{3} / 12\right)+\left(b t_{f}^{3} /\right.\right.$ 6) $\left.+2 b t_{f}\left(\left(h-t_{f}\right) / 2\right)^{2}\right)$

subject to $g(\vec{x})=2 b t_{w}+t_{w}\left(h-2 t_{f}\right) \leq 0$

Variable range:

$$
\begin{aligned}
& 10 \leq x_{1} \leq 50 \\
& 10 \leq x_{2} \leq 80 \\
& 0.9 \leq x_{3} \leq 5 \\
& 0.9 \leq x_{4} \leq 5
\end{aligned}
$$

Wang used the ARSM [174] method to solve the model and obtained a minimum vertical deflection of 0.0157 . The minimum vertical deflection optimized by the improved method IARSM is 0.0131. Gandomi et al. [175] utilized CS to decrease the minimum vertical deflection to 0.0130747 . Cheng and Prayogo [176] used SOS to obtain deflection the value of 0.0130741 . Table 9 demonstrates that the I-beam's vertical deflection obtained by the MEWOA algorithm is 0.0130741 , and the experimental result is better than other comparison methods. 
TABLE 8: Comparison results of MEWOA and other algorithms on the welded beam case.

\begin{tabular}{lccccc}
\hline \multirow{2}{*}{ Technique } & \multicolumn{2}{c}{ Best variables } & $t$ & $b$ & Best cost \\
& $h$ & $L$ & 9.11343 & 0.206754 \\
MEWOA & 0.1885 & 3.471 & 9.004233 & 0.207241 & 1.720001 \\
RO [171] & 0.203687 & 3.528467 & 9.033498 & 0.206179 & 1.735344 \\
CDE [173] & 0.203137 & 3.542998 & 9.03662 & 0.20573 & 1.733462 \\
IHS [170] & 0.20573 & 3.47049 & & & 1.7248 \\
\hline
\end{tabular}

TABLE 9: Comparison results of MEWOA and other algorithms on the I-beam case.

\begin{tabular}{lccccc}
\hline \multirow{2}{*}{ Algorithm } & \multicolumn{3}{c}{ Best variables } & $t_{f}$ & Optimum vertical \\
& $b$ & $h$ & $t_{w}$ & 2.32179 & 0.0130741 \\
\hline MEWOA & 50 & 80 & 0.9 & 2.31 & 0.0157 \\
ARSM [174] & 37.05 & 80 & 1.71 & 2.40 & 0.0131 \\
IARSM [174] & 48.42 & 79.99 & 0.90 & 2.321675 & 0.0130747 \\
CS [175] & 50 & 80 & 0.9 & 2.32179 & 0.0130741 \\
SOS [176] & 50 & 80 &
\end{tabular}

\section{Conclusions and Future Works}

This article presents an enhanced MEWOA by integrating the opposition-based learning mechanism and MFO algorithm to enhance the balance between exploration and exploitation of the original WOA. Firstly, the MEWOA was estimated and compared with the basic algorithms in different dimensions to verify its effectiveness. Moreover, the devised MEWOA was also compared with six metaheuristic algorithms and five advanced algorithms to demonstrate its superiority. The experimental results proved that MEWOA has achieved much better performance than the original one. The convergence accuracy and scalability of MEWOA also have been improved a lot. The MEWOA can also effectively solve practical engineering problems. The results have shown that MEWOA can achieve a good balance between the exploration and exploitation ability and solve the constraint problem effectively.

In future work, we plan to improve the WOA in a deeper way from the principle of WOA. And the MEWOA can also be extended to a multiobjective version or binary version for other optimization tasks.

\section{Data Availability}

The data involved in this study are all public data, which can be downloaded through public channels.

\section{Conflicts of Interest}

The authors declare that they have no conflicts of interest.

\section{Acknowledgments}

This research was funded by the Foundation of Jilin Educational Committee (JJKH20210750KJ), Guangdong Natural Science Foundation (2018A030313339), Scientific Research Team Project of Shenzhen Institute of Information Technology (SZIIT2019KJ022), and Taif University Researchers
Supporting Project Number (TURSP-2020/125), Taif University, Taif, Saudi Arabia. Thanks are due to the efforts of Ali Asghar Heidari (https://aliasgharheidari.com) during the preparation of this research.

\section{References}

[1] S. Qu, Y. Han, Z. Wu, and H. Raza, "Consensus modeling with asymmetric cost based on data-driven robust optimization," Group Decision and Negotiation, vol. 7, pp. 1-38, 2020.

[2] B. Cao, J. Zhao, Y. Gu, Y. Ling, and X. Ma, “Applying graphbased differential grouping for multiobjective large-scale optimization," Swarm and Evolutionary Computation, vol. 53, Article ID 100626, 2020.

[3] B. Cao, S. Fan, J. Zhao, P. Yang, K. Muhammad, and M. Tanveer, "Quantum-enhanced multiobjective large-scale optimization via parallelism," Swarm and Evolutionary Computation, vol. 57, Article ID 100697, 2020.

[4] B. Cao, "Multiobjective 3-D topology optimization of nextgeneration wireless data center network," IEEE Transactions on Industrial Informatics, vol. 16, no. 5, pp. 3597-3605, 2019.

[5] H. Huang, X. a. Feng, S. Zhou et al., "A new fruit fly optimization algorithm enhanced support vector machine for diagnosis of breast cancer based on high-level features," BMC Bioinformatics, vol. 20, no. S8, 2019.

[6] B. Cao, "Hybrid Microgrid many-objective sizing optimization with fuzzy decision," IEEE Transactions on Fuzzy Systems, vol. 28, no. 11, 2020.

[7] B. Cao, X. Wang, W. Zhang, H. Song, and Z. Lv, "A manyobjective optimization model of industrial internet of things based on private blockchain," IEEE Network, vol. 34, no. 5, pp. 78-83, 2020.

[8] W. Deng, "An improved quantum-inspired differential evolution algorithm for deep belief network," IEEE Transactions on Instrumentation \& Measurement, vol. 69, 2020.

[9] H. Zhao, "Performance prediction using high-order differential mathematical morphology gradient spectrum entropy and extreme learning machine," IEEE Transactions on Instrumentation \& Measurement, vol. 69, 2019. 
[10] W. Deng, "A novel gate resource allocation method using improved PSO-based QEA," IEEE Transactions on Intelligent Transportation Systems, vol. 19, 2020.

[11] H. Chen, A. A. Heidari, H. Chen, M. Wang, Z. Pan, and A. H. Gandomi, "Multi-population differential evolutionassisted Harris hawks optimization: framework and case studies," Future Generation Computer Systems, vol. 111, pp. 175-198, 2020.

[12] Y. Xu, H. Chen, J. Luo, Q. Zhang, S. Jiao, and X. Zhang, "Enhanced Moth-flame optimizer with mutation strategy for global optimization," Information Sciences, vol. 492, pp. 181-203, 2019.

[13] X. Xu and H.-l. Chen, "Adaptive computational chemotaxis based on field in bacterial foraging optimization," Soft Computing, vol. 18, no. 4, pp. 797-807, 2014.

[14] H. Chen, Y. Xu, M. Wang, and X. Zhao, "A balanced whale optimization algorithm for constrained engineering design problems," Applied Mathematical Modelling, vol. 71, pp. 45-59, 2019.

[15] H. Yu, N. Zhao, P. Wang, H. Chen, and C. Li, "Chaos-enhanced synchronized bat optimizer," Applied Mathematical Modelling, vol. 77, pp. 1201-1215, 2020.

[16] H. L. Chen, M. J. Wang, and X. H. Zhao, "A multi-strategy enhanced sine cosine algorithm for global optimization and constrained practical engineering problems," Applied Mathematics and Computation, vol. 369, 2020.

[17] X. Zhang, Y. Xu, C. Yu et al., "Gaussian mutational chaotic fruit fly-built optimization and feature selection," Expert Systems with Applications, vol. 141, Article ID 112976, 2020.

[18] M. A. Al-Betar, "Survival exploration strategies for Harris hawks optimizer," Expert Systems with Applications, vol. 168, 2020.

[19] J. Luo, H. Chen, Q. zhang, Y. Xu, H. Huang, and X. Zhao, "An improved grasshopper optimization algorithm with application to financial stress prediction," Applied Mathematical Modelling, vol. 64, pp. 654-668, 2018.

[20] H. L. Chen, "Efficient multi-population outpost fruit flydriven optimizers: framework and advances in support vector machines," Expert Systems with Applications, vol. 142, 2020.

[21] H. Chen, C. Yang, A. A. Heidari, and X. Zhao, "An efficient double adaptive random spare reinforced whale optimization algorithm," Expert Systems with Applications, vol. 154, Article ID 113018, 2020.

[22] A. A. Heidari, I. Aljarah, H. Faris, H. Chen, J. Luo, and S. Mirjalili, "An enhanced associative learning-based exploratory whale optimizer for global optimization," Neural Computing \& Applications, vol. 32, no. 9, pp. 5185-5211, 2019.

[23] L. Shen, H. Chen, Z. Yu et al., "Evolving support vector machines using fruit fly optimization for medical data classification," Knowledge-Based Systems, vol. 96, pp. 61-75, 2016.

[24] Y. Zhang, R. Liu, X. Wang, H. Chen, and C. Li, "Boosted binary Harris hawks optimizer and feature selection," Engineering with Computers, vol. 57, 2020.

[25] X. Zhao, D. Li, B. Yang, C. Ma, Y. Zhu, and H. Chen, "Feature selection based on improved ant colony optimization for online detection of foreign fiber in cotton," Applied Soft Computing, vol. 24, pp. 585-596, 2014.

[26] M. Wang and H. Chen, "Chaotic multi-swarm whale optimizer boosted support vector machine for medical diagnosis," Applied Soft Computing, vol. 88, Article ID 105946, 2020.
[27] X. Zhao, D. Li, B. Yang et al., "A two-stage feature selection method with its application," Computers \& Electrical Engineering, vol. 47, pp. 114-125, 2015.

[28] C. Li, L. Hou, B. Y. Sharma et al., "Developing a new intelligent system for the diagnosis of tuberculous pleural effusion," Computer Methods and Programs in Biomedicine, vol. 153, pp. 211-225, 2018.

[29] M. Wang, H. Chen, B. Yang et al., "Toward an optimal kernel extreme learning machine using a chaotic moth-flame optimization strategy with applications in medical diagnoses," Neurocomputing, vol. 267, pp. 69-84, 2017.

[30] J. Xia, H. Chen, Q. Li et al., "Ultrasound-based differentiation of malignant and benign thyroid Nodules: an extreme learning machine approach," Computer Methods and Programs in Biomedicine, vol. 147, pp. 37-49, 2017.

[31] H.-L. Chen, G. Wang, C. Ma, Z.-N. Cai, W.-B. Liu, and S.-J. Wang, "An efficient hybrid kernel extreme learning machine approach for early diagnosis of Parkinson's disease," Neurocomputing, vol. 184, pp. 131-144, 2016.

[32] L. Hu, G. Hong, J. Ma, X. Wang, and H. Chen, "An efficient machine learning approach for diagnosis of paraquat-poisoned patients," Computers in Biology and Medicine, vol. 59, pp. 116-124, 2015.

[33] S.-J. Wang, H.-L. Chen, W.-J. Yan, Y.-H. Chen, and X. Fu, "Face recognition and micro-expression recognition based on discriminant tensor subspace analysis plus extreme learning machine," Neural Processing Letters, vol. 39, no. 1, pp. 25-43, 2014.

[34] X. Zhao, X. Zhang, Z. Cai et al., "Chaos enhanced grey wolf optimization wrapped ELM for diagnosis of paraquat-poisoned patients," Computational Biology and Chemistry, vol. 78, pp. 481-490, 2019.

[35] Y. Zhang, "Towards augmented kernel extreme learning models for bankruptcy prediction: algorithmic behavior and comprehensive analysis," Neurocomputing, vol. 430, 2020.

[36] C. Yu, M. Chen, K. Cheng et al., "SGOA: annealing-behaved grasshopper optimizer for global tasks," Engineering with Computers, vol. 26, 2021.

[37] Z. Cai, J. Gu, J. Luo et al., "Evolving an optimal kernel extreme learning machine by using an enhanced grey wolf optimization strategy," Expert Systems with Applications, vol. 138, Article ID 112814, 2019.

[38] M. Wang, H. Chen, H. Li et al., "Grey wolf optimization evolving kernel extreme learning machine: application to bankruptcy prediction," Engineering Applications of Artificial Intelligence, vol. 63, pp. 54-68, 2017.

[39] X. Wang, H. Chen, A. A. Heidari et al., "Multi-population following behavior-driven fruit fly optimization: a Markov chain convergence proof and comprehensive analysis," Knowledge-Based Systems, vol. 210, Article ID 106437, 2020.

[40] J. Tu, "Evolutionary biogeography-based Whale optimization methods with communication structure: towards measuring the balance," Knowledge-Based Systems, vol. 212, Article ID 106642, 2020.

[41] S. Song, "Dimension decided Harris hawks optimization with Gaussian mutation: balance analysis and diversity patterns," Knowledge-Based Systems, vol. 215, Article ID 106425, 2020.

[42] X. Liang, Z. Cai, M. Wang, X. Zhao, H. Chen, and C. Li, "Chaotic oppositional sine-cosine method for solving global optimization problems," Engineering with Computers, vol. 7, 2020. 
[43] A. F. Ba, H. Huang, M. Wang et al., "Levy-based antlioninspired optimizers with orthogonal learning scheme," Engineering with Computers, vol. 21, 2020.

[44] J. Yan, W. Pu, S. Zhou, H. Liu, and M. S. Greco, "Optimal resource allocation for asynchronous multiple targets tracking in heterogeneous radar networks," IEEE Transactions on Signal Processing, vol. 68, pp. 4055-4068, 2020.

[45] C. Li, L. Sun, Z. Xu, X. Wu, T. Liang, and W. Shi, "Experimental investigation and error analysis of high precision fbg displacement sensor for structural health monitoring," International Journal of Structural Stability and Dynamics, vol. 20, no. 6, Article ID 2040011, 2020.

[46] L. Sun, C. Li, C. Zhang, T. Liang, and Z. Zhao, "The strain transfer mechanism of fiber bragg grating sensor for extra large strain monitoring," Sensors, vol. 19, no. 8, Article ID 1851, 2019.

[47] C. Zhang, "Fibre Bragg grating sensor-based damage response monitoring of an asymmetric reinforced concrete shear wall structure subjected to progressive seismic loads," Structural Control and Health Monitoring, vol. 26, no. 3, Article ID e2307, 2019.

[48] L. Sun, C. Li, C. Zhang, Z. Su, and C. Chen, "Early monitoring of rebar corrosion evolution based on FBG sensor," International Journal of Structural Stability and Dynamics, vol. 18, no. 8, Article ID 1840001, 2018.

[49] C. Cai, "Selective laser melting of near- $\alpha$ titanium alloy Ti$6 \mathrm{Al}-2 \mathrm{Zr}-1 \mathrm{Mo}-1 \mathrm{~V}$ : parameter optimization, heat treatment and mechanical performance," Journal of Materials Science \& Technology, vol. 57, 2020.

[50] C. Cai, "Hot isostatic pressing of a near $\alpha$-Ti alloy: temperature optimization, microstructural evolution and mechanical performance evaluation," Materials Science and Engineering: A, vol. 802, Article ID 140426, 2020.

[51] H. Kordestani and C. Zhang, "E-direct use of the savitzkygolay filter to develop an output-only trend line-based damage detection method," Sensors, vol. 20, no. 7, Article ID 1983, 2020.

[52] A. A. Mousavi, C. Zhang, S. F. Masri, and G. Gholipour, "Structural damage localization and quantification based on a CEEMDAN hilbert transform neural network approach: a model steel truss bridge case study," Sensors, vol. 20, no. 5, Article ID 1271, 2020.

[53] X. Wang, Y. Liu, and K. R. Choo, "Fault tolerant multi-subset aggregation scheme for smart grid," IEEE Transactions on Industrial Informatics, vol. 171 page, 2020.

[54] B. Wang, B. F. Zhang, X. W. Liu, and F. C. Zou, "Novel infrared image enhancement optimization algorithm combined with DFOCS," Optik, vol. 224, Article ID 165476, 2020.

[55] Q. Zhu, "Research on road traffic situation awareness system based on image big data," IEEE Intelligent Systems, vol. 35, no. 1, pp. 18-26, 2019.

[56] Q. Jiang, "Unified no-reference quality assessment of singly and multiply distorted stereoscopic images," IEEE Transactions on Image Processing, vol. 28, no. 4, pp. 1866-1881, 2018.

[57] M. Xu, C. Li, S. Zhang, and P. L. Callet, "State-of-the-Art in $360^{\circ}$ video/image processing: perception, assessment and compression," IEEE Journal of Selected Topics in Signal Processing, vol. 14, no. 1, pp. 5-26, 2020.

[58] M. Yang and A. Sowmya, "An underwater color image quality evaluation metric," IEEE Transactions on Image Processing, vol. 24, no. 12, pp. 6062-6071, 2015.
[59] B. Wang, B. Zhang, and X. Liu, "An image encryption approach on the basis of a time delay chaotic system," Optik, vol. 225, Article ID 165737, 2021.

[60] S. Hinojosa, D. Oliva, E. Cuevas, G. Pajares, D. Zaldivar, and M. Pérez-Cisneros, "Reducing overlapped pixels: a multiobjective color thresholding approach," Soft Computing, vol. 24, no. 9, pp. 6787-6807, 2020.

[61] D. Liu, S. Wang, D. Huang, G. Deng, F. Zeng, and H. Chen, "Medical image classification using spatial adjacent histogram based on adaptive local binary patterns," Computers in Biology and Medicine, vol. 72, pp. 185-200, 2016.

[62] D. Zhao, "Ant colony optimization with horizontal and vertical crossover search: fundamental visions for multithreshold image segmentation," Expert Systems with Applications, vol. 167, Article ID 114122, 2020.

[63] D. Zhao, "Chaotic random spare ant colony optimization for multi-threshold image segmentation of 2D Kapur entropy," Knowledge-Based Systems, vol. 216, Article ID 106510, 2020.

[64] S. Li, H. Chen, M. Wang, A. A. Heidari, and S. Mirjalili, "Slime mould algorithm: a new method for stochastic optimization," Future Generation Computer Systems, vol. 111, pp. 300-323, 2020.

[65] A. A. Heidari, S. Mirjalili, H. Faris, I. Aljarah, M. Mafarja, and H. Chen, "Harris hawks optimization: algorithm and applications," Future Generation Computer Systems, vol. 97, pp. 849-872, 2019.

[66] R. Salgotra and U. Singh, "The naked mole-rat algorithm," Neural Computing \& Applications, vol. 31, no. 12, pp. 8837-8857, 2019.

[67] Y. Yang, "Hunger games search: visions, conception, implementation, deep analysis, perspectives, and towards performance shifts," Expert Systems with Applications, vol. 177, 2021.

[68] G.-G. Wang, "Moth search algorithm: a bio-inspired metaheuristic algorithm for global optimization problems," Memetic Computing, vol. 10, no. 2, pp. 151-164, 2018.

[69] Y. Feng, S. Deb, G.-G. Wang, and A. H. Alavi, "Monarch butterfly optimization: a comprehensive review," Expert Systems with Applications, vol. 168, Article ID 114418, 2021.

[70] G.-G. Wang, A. H. Gandomi, A. H. Alavi, and D. Gong, "A comprehensive review of krill herd algorithm: variants, hybrids and applications," Artificial Intelligence Review, vol. 51, no. 1, pp. 119-148, 2019.

[71] N. Gao, D. Luo, B. Cheng, and H. Hou, "Teaching-learningbased optimization of a composite metastructure in the 0-10 $\mathrm{kHz}$ broadband sound absorption range," Journal of the Acoustical Society of America, vol. 148, no. 2, pp. EL125EL129, 2020.

[72] G. Sun, "An adaptive differential evolution with combined strategy for global numerical optimization," Soft Computing, vol. 24, pp. 1-20, 2019.

[73] J. Liu, C. Wu, G. Wu, and X. Wang, "A novel differential search algorithm and applications for structure design," Applied Mathematics and Computation, vol. 268, pp. 246269, 2015.

[74] S. Xu, "Computer vision techniques in construction: a critical review," Archives of Computational Methods in Engineering, vol. 28, pp. 3383-3397, 2020.

[75] B. Cao, "Security-aware industrial wireless sensor network deployment optimization," IEEE Transactions on Industrial Informatics, vol. 16, no. 8, pp. 5309-5316, 2019.

[76] C. Wu, P. Wu, J. Wang, R. Jiang, M. Chen, and X. Wang, "Ontological knowledge base for concrete bridge 
rehabilitation project management," Automation in Construction, vol. 121, Article ID 103428, 2021.

[77] T. Qiu, X. Shi, J. Wang et al., "Deep learning: a rapid and efficient route to automatic metasurface design," Advanced Science, vol. 6, no. 12, Article ID 1900128, 2019.

[78] T. Li, M. Xu, C. Zhu, R. Yang, Z. Wang, and Z. Guan, "A deep learning approach for multi-frame in-loop filter of HEVC," IEEE Transactions on Image Processing, vol. 28, no. 11, pp. 5663-5678, 2019.

[79] H. Chen, A. Chen, L. Xu et al., "A deep learning CNN architecture applied in smart near-infrared analysis of water pollution for agricultural irrigation resources," Agricultural Water Management, vol. 240, Article ID 106303, 2020.

[80] Q. Jiang, G. Wang, S. Jin, Y. Li, and Y. Wang, "Predicting human microRNA-disease associations based on support vector machine," International Journal of Data Mining and Bioinformatics, vol. 8, no. 3, pp. 282-293, 2013.

[81] K. Qu, L. Wei, and Q. Zou, "A review of DNA-binding proteins prediction methods," Current Bioinformatics, vol. 14, no. 3, pp. 246-254, 2019.

[82] C. Wu, P. Wu, J. Wang, R. Jiang, M. Chen, and X. Wang, "Critical review of data-driven decision-making in bridge operation and maintenance," Structure and Infrastructure Engineering, vol. 21, pp. 1-24, 2020.

[83] S. Liu, "A variable weight-based hybrid approach for multiattribute group decision making under interval valued intuitionistic fuzzy sets," International Journal of Intelligent Systems, vol. 39, 2020.

[84] S. Liu, F. T. S. Chan, and W. Ran, "Decision making for the selection of cloud vendor: an improved approach under group decision-making with integrated weights and objective/subjective attributes," Expert Systems with Applications, vol. 55, pp. 37-47, 2016.

[85] S. Mirjalili and A. Lewis, "The whale optimization algorithm," Advances in Engineering Software, vol. 95, pp. 51-67, 2016.

[86] W. L. Wang, W. K. Li, Z. Wang, and L. Li, “Opposition-based multi-objective whale optimization algorithm with global grid ranking," Neurocomputing, vol. 341, pp. 41-59, 2019.

[87] R. Salgotra, U. Singh, and S. Saha, "On some improved versions of whale optimization algorithm," Arabian Journal for Science and Engineering, vol. 44, no. 11, pp. 9653-9691, 2019.

[88] Y. Sun, T. Yang, and Z. Liu, "A whale optimization algorithm based on quadratic interpolation for high-dimensional global optimization problems," Applied Soft Computing, vol. 85, 2019.

[89] R. K. Agrawal, B. Kaur, and S. Sharma, "Quantum based whale optimization algorithm for wrapper feature selection," Applied Soft Computing, vol. 89, 2020.

[90] A. G. Hussien, "New binary whale optimization algorithm for discrete optimization problems," Engineering Optimization, vol. 52, no. 6, 2019.

[91] J. Luo, H. Chen, A. A. Heidari, Y. Xu, Q. Zhang, and C. Li, "Multi-strategy boosted mutative whale-inspired optimization approaches," Applied Mathematical Modelling, vol. 73, pp. 109-123, 2019.

[92] K. Shi, Y. Tang, X. Liu, and S. Zhong, "Non-fragile sampleddata robust synchronization of uncertain delayed chaotic Lurie systems with randomly occurring controller gain fluctuation," ISA Transactions, vol. 66, pp. 185-199, 2017.

[93] T. Wu, L. Xiong, J. Cheng, and X. Xie, "New results on stabilization analysis for fuzzy semi-Markov jump chaotic systems with state quantized sampled-data controller," Information Sciences, vol. 521, pp. 231-250, 2020.

[94] T. Wu, "New stabilization results for semi-markov chaotic systems with fuzzy sampled-data control," Complexity, vol. 2019, 2019.

[95] Y. Sun, X. Wang, Y. Chen, and Z. Liu, "A modified whale optimization algorithm for large-scale global optimization problems," Expert Systems with Applications, vol. 114, pp. 563-577, 2018.

[96] F. Hemasian-Etefagh and F. Safi-Esfahani, "Group-based whale optimization algorithm," Soft Computing, vol. 24, no. 5, pp. 3647-3673, 2020.

[97] M. A. Elaziz and S. Mirjalili, "A hyper-heuristic for improving the initial population of whale optimization algorithm," Knowledge-Based Systems, vol. 172, pp. 42-63, 2019.

[98] W. Guo, "An improved whale optimization algorithm for forecasting water resources demand," Applied Soft Computing, vol. 86, 2020.

[99] S. T. Revathi, N. Ramaraj, and S. Chithra, "Brain storm-based whale optimization algorithm for privacy-protected data publishing in cloud computing," Cluster Computing-the Journal of Networks Software Tools and Applications, vol. 22, no. 2, pp. S3521-S3530, 2019.

[100] S. Gong, W. Gao, and F. Abza, "Brain tumor diagnosis based on artificial neural network and a chaos whale optimization algorithm," Computational Intelligence, vol. 36, no. 1, pp. 259-275, 2020.

[101] N. Zhang, Y. X Cai, Y. Y Wang, Y. T Tian, X. L Wang, and B Badami, "Skin cancer diagnosis based on optimized convolutional neural network," Artificial Intelligence in Medicine, vol. 102, Article ID 101756, 2020.

[102] G. Xiong, J. Zhang, D. Shi, and Y. He, "Parameter extraction of solar photovoltaic models using an improved whale optimization algorithm," Energy Conversion and Management, vol. 174, pp. 388-405, 2018.

[103] M. Petrović, Z. Miljkovic, and A. Jokic, "A novel methodology for optimal single mobile robot scheduling using whale optimization algorithm," Applied Soft Computing, vol. 81, 2019.

[104] L.-L. Li, J. Sun, M.-L. Tseng, and Z.-G. Li, "Extreme learning machine optimized by whale optimization algorithm using insulated gate bipolar transistor module aging degree evaluation," Expert Systems with Applications, vol. 127, pp. 5867, 2019.

[105] S. Akyol and B. Alatas, "Sentiment classification within online social media using whale optimization algorithm and social impact theory based optimization," Physica A-Statistical Mechanics and Its Applications, vol. 540, 2020.

[106] W. Qiao, "Short-term natural gas consumption prediction based on Volterra adaptive filter and improved whale optimization algorithm," Engineering Applications of Artificial Intelligence, vol. 87, 2020.

[107] X. Ye, "Modified whale optimization algorithm for solar cell and PV module parameter identification," Complexity, vol. 2021, Article ID 8878686, , 2021.

[108] S. Mirjalili, "Moth-flame optimization algorithm: a novel nature-inspired heuristic paradigm," Knowledge-Based Systems, vol. 89, pp. 228-249, 2015.

[109] Y. Xu, H. Chen, A. A. Heidari et al., "An efficient chaotic mutative moth-flame-inspired optimizer for global optimization tasks," Expert Systems with Applications, vol. 129, pp. 135-155, 2019.

[110] S. Yang, "Scalable digital neuromorphic architecture for large-scale biophysically meaningful neural network with 
multi-compartment neurons," IEEE transactions on neural networks and learning systems, vol. 31, no. 1, pp. 148-162, 2019.

[111] T. Ni, H. Chang, T. Song et al., "Non-intrusive online distributed pulse shrinking-based interconnect testing in $2.5 \mathrm{D}$ IC," IEEE Transactions on Circuits and Systems II: Express Briefs, vol. 67, no. 11, pp. 2657-2661, 2020.

[112] H. Zhang, Z. Qiu, J. Cao, M. Abdel-Aty, and L. Xiong, "Event-triggered synchronization for Neutral-Type semiMarkovian neural networks with partial mode-dependent time-varying delays," IEEE Transactions on Neural Networks and Learning Systems, vol. 31, no. 11, pp. 4437-4450, 2020.

[113] J. Derrac, S. García, D. Molina, and F. Herrera, “A practical tutorial on the use of nonparametric statistical tests as a methodology for comparing evolutionary and swarm intelligence algorithms," Swarm and Evolutionary Computation, vol. 1, no. 1, pp. 3-18, 2011.

[114] S. García, A. Fernández, J. Luengo, and F. Herrera, "Advanced nonparametric tests for multiple comparisons in the design of experiments in computational intelligence and data mining: experimental analysis of power," Information Sciences, vol. 180, no. 10, pp. 2044-2064, 2010.

[115] H. R. Tizhoosh, "Opposition-based learning: a new scheme for machine intelligence," in Proceedings of the International Conference on Computational Intelligence for Modelling, Control and Automation and International Conference on Intelligent Agents, Web Technologies and Internet Commerce (CIMCA-IAWTIC'06), Vienna, Austria, November 2005.

[116] N. Awad, "Problem definitions and evaluation criteria for the CEC 2017 special session and competition on single objective real-parameter numerical optimization," Technical Report, 2016.

[117] V. Bhargava, S. E. K. Fateen, and A. Bonilla-Petriciolet, "Cuckoo Search: a new nature-inspired optimization method for phase equilibrium calculations," Fluid Phase Equilibria, vol. 337, pp. 191-200, 2013.

[118] S. Saremi, S. Mirjalili, and A. Lewis, "Grasshopper optimisation algorithm: theory and application," Advances in Engineering Software, vol. 105, pp. 30-47, 2017.

[119] N. Lynn and P. N. Suganthan, "Heterogeneous comprehensive learning particle swarm optimization with enhanced exploration and exploitation," Swarm and Evolutionary Computation, vol. 24, pp. 11-24, 2015.

[120] M. G. H. Omran, A. Salman, and A. P. Engelbrecht, "Selfadaptive differential evolution," in Proceedings of the Computational Intelligence and Security, pp. 192-199, Guangzhou, China, December 2005.

[121] J. Jingqiao Zhang and A. C. Sanderson, "JADE: adaptive differential evolution with optional external archive," IEEE Transactions on Evolutionary Computation, vol. 13, no. 5, pp. 945-958, 2009.

[122] J. J. Liang, A. K. Qin, P. N. Suganthan, and S. Baskar, "Comprehensive learning particle swarm optimizer for global optimization of multimodal functions," IEEE Transactions on Evolutionary Computation, vol. 10, no. 3, pp. 281-295, 2006.

[123] R. Tanabe and A. S. Fukunaga, "Improving the search performance of SHADE using linear population size reduction," in Proceedings of the Evolutionary Computation, Beijing, China, July 2014.

[124] N. H. Awad, M. Z. Ali, and P. N. Suganthan, "Ensemble sinusoidal differential covariance matrix adaptation with Euclidean neighborhood for solving CEC2017 benchmark problems," in Proceedings of the 2017 IEEE Congress on
Evolutionary Computation (CEC), Donostia, Spain, June 2017.

[125] X. Fei, J. Wang, S. Ying, Z. Hu, and J. Shi, "Projective parameter transfer based sparse multiple empirical kernel learning Machine for diagnosis of brain disease," Neurocomputing, vol. 413, pp. 271-283, 2020.

[126] H. Zhao, X. Qiu, W. Lu, H. Huang, and X. Jin, "High-quality retinal vessel segmentation using generative adversarial network with a large receptive field," International Journal of Imaging Systems and Technology, vol. 30, no. 3, pp. 828-842, 2020.

[127] T. Wang, "Video deblurring via spatiotemporal pyramid network and adversarial gradient prior," Computer Vision and Image Understanding, vol. 203, Article ID 103135, 2020.

[128] X. Zhang, R. Jiang, T. Wang, P. Huang, and L. Zhao, "Attention-based interpolation network for video deblurring," Neurocomputing, vol. 453, pp. 865-875, 2021.

[129] X. Zhang, R. Jiang, T. Wang, and J. Wang, "Recursive neural network for video deblurring," IEEE Transactions on Circuits and Systems for Video Technology, vol. 31, no. 8, pp. 30253036, 2021.

[130] X. Zhang, T. Wang, J. Wang, G. Tang, and L. Zhao, "Pyramid channel-based feature attention network for image dehazing," Computer Vision and Image Understanding, vol. 198, Article ID 103003, 2020.

[131] Y. Li, W.-G. Cui, H. Huang, Y.-Z. Guo, K. Li, and T. Tan, "Epileptic seizure detection in EEG signals using sparse multiscale radial basis function networks and the Fisher vector approach," Knowledge-Based Systems, vol. 164, pp. 96-106, 2019.

[132] Y. Li, J. Liu, Z. Tang, and B. Lei, "Deep spatial-temporal feature fusion from adaptive dynamic functional connectivity for MCI identification," IEEE Transactions on Medical Imaging, vol. 39, no. 9, pp. 2818-2830, 2020.

[133] X. Zhang, M. Fan, D. Wang, P. Zhou, and D. Tao, “Top-k feature selection framework using robust 0-1 integer programming," IEEE Transactions on Neural Networks and Learning Systems, vol. 32, no. 7, pp. 3005-3019, 2021.

[134] X. Wu, X. Xu, J. Liu, H. Wang, B. Hu, and F. Nie, "Supervised feature selection with orthogonal regression and feature weighting," IEEE Transactions on Neural Networks and Learning Systems, vol. 32, no. 5, pp. 1831-1838, 2021.

[135] N. Gu, M. Fan, L. Du, and D. Ren, "Efficient sequential feature selection based on adaptive eigenspace model," Neurocomputing, vol. 161, pp. 199-209, 2015.

[136] M. Fan, N. Gu, H. Qiao, and B. Zhang, "Dimensionality reduction: an interpretation from manifold regularization perspective," Information Sciences, vol. 277, pp. 694-714, 2014.

[137] H. M. Ridha, C. Gomes, H. Hizam, M. Ahmadipour, A. A. Heidari, and H. Chen, "Multi-objective optimization and multi-criteria decision-making methods for optimal design of standalone photovoltaic system: a comprehensive review," Renewable and Sustainable Energy Reviews, vol. 135, Article ID 110202, 2021.

[138] H. Chen, S. Jiao, M. Wang, A. A. Heidari, and X. Zhao, "Parameters identification of photovoltaic cells and modules using diversification-enriched Harris hawks optimization with chaotic drifts," Journal of Cleaner Production, vol. 244, Article ID 118778, 2020.

[139] A. Abbassi, R. Abbassi, A. A. Heidari et al., "Parameters identification of photovoltaic cell models using enhanced exploratory salp chains-based approach," Energy, vol. 198, Article ID 117333, 2020. 
[140] H. Zhang, A. A. Heidari, M. Wang, L. Zhang, H. Chen, and C. Li, "Orthogonal Nelder-Mead moth flame method for parameters identification of photovoltaic modules," Energy Conversion and Management, vol. 211, Article ID 112764, 2020.

[141] Y. Liu, G. Chong, A. A. Heidari et al., "Horizontal and vertical crossover of Harris hawk optimizer with NelderMead simplex for parameter estimation of photovoltaic models," Energy Conversion and Management, vol. 223, Article ID 113211, 2020.

[142] J. Li, C. Chen, H. Chen, and C. Tong, "Towards contextaware social recommendation via individual trust," Knowledge-Based Systems, vol. 127, pp. 58-66, 2017.

[143] J. Li and J. Lin, "A probability distribution detection based hybrid ensemble QoS prediction approach," Information Sciences, vol. 519, pp. 289-305, 2020.

[144] J. Li, X.-L. Zheng, S.-T. Chen, W.-W. Song, and D.-r. Chen, "An efficient and reliable approach for quality-of-serviceaware service composition," Information Sciences, vol. 269, pp. 238-254, 2014.

[145] Q. Li, X. Wu, and T. Liu, "Differentiable neural architecture search for optimal spatial/temporal brain function network decomposition," Medical Image Analysis, vol. 69, Article ID 101974, 2021.

[146] Z. Zhu, Z. Zhen, X. Wu, and S. Li, "Estimating functional connectivity by integration of inherent brain function activity pattern priors," IEEE/ACM Transactions on Computational Biology and Bioinformatics, vol. 951 page, 2020.

[147] X. Li, H. Huang, H. Zhao, Y. Wang, and M. Hu, "Learning a convolutional neural network for propagation-based stereo image segmentation," The Visual Computer, vol. 36, no. 1, pp. 39-52, 2020.

[148] X. Li, H. Zhao, H. Huang, Z. Hu, and L. Xiao, "Interactive image recoloring by combining global and local optimization," Multimedia Tools and Applications, vol. 75, no. 11, pp. 6431-6443, 2016.

[149] H. Huang, X. Li, H. Zhao, G. Nie, Z. Hu, and L. Xiao, "Manifold-preserving image colorization with nonlocal estimation," Multimedia Tools and Applications, vol. 74, no. 18, pp. 7555-7568, 2015.

[150] T. Wang, "Haze concentration adaptive network for image dehazing," Neurocomputing, vol. 439, 2021

[151] P. Huang, "Self-filtering image dehazing with self-supporting module," Neurocomputing, vol. 432, pp. 57-69, 2020.

[152] X. Zhang, T. Wang, W. Luo, and P. Huang, "Multi-level fusion and attention-guided CNN for image dehazing," IEEE Transactions on Circuits and Systems for Video Technology, vol. 25, no. 1, 2020.

[153] L. Zhang, Y. Zou, W. Wang, Z. Jin, Y. Su, and H. Chen, "Resource allocation and trust computing for blockchainenabled edge computing system," Computers \& Security, vol. 105, Article ID 102249, 2021.

[154] L. Zhang, "A covert communication method using special bitcoin addresses generated by vanitygen," Computers, Materials le Continua, vol. 65, no. 1, pp. 597-616, 2020.

[155] L. Zhang, Z. Zhang, W. Wang, Z. Jin, Y. Su, and H. Chen, "Research on a covert communication model realized by using smart contracts in blockchain environment," IEEE Systems Journal, vol. 12, no. 1, 2021.

[156] Y. Wei, H. Lv, M. Chen et al., "Predicting entrepreneurial intention of students: an extreme learning machine with Gaussian barebone Harris hawks optimizer," IEEE Access, vol. 8, pp. 76841-76855, 2020.
[157] W. Zhu, C. Ma, X. Zhao et al., "Evaluation of sino foreign cooperative education project using orthogonal sine cosine optimized kernel extreme learning machine," IEEE Access, vol. 8, pp. 61107-61123, 2020.

[158] A. Lin, Q. Wu, A. A. Heidari et al., "Predicting intentions of students for master programs using a chaos-induced sine cosine-based fuzzy K-nearest neighbor classifier," IEEE Access, vol. 7, pp. 67235-67248, 2019.

[159] J. Tu, A. Lin, H. Chen, Y. Li, and C. Li, "Predict the entrepreneurial intention of fresh graduate students based on an adaptive support vector machine framework," Mathematical Problems in Engineering, vol. 201916 pages, 2019.

[160] Y. Wei, N. Ni, D. Liu et al., "An improved grey wolf optimization strategy enhanced SVM and its application in predicting the second major," Mathematical Problems in Engineering, vol. 201712 pages, 2017.

[161] Y. Li, X. Liu, S. Zhang, and X. Ye, "Human articulated body recognition method in high-resolution monitoring images," Neurocomputing, vol. 181, pp. 116-121, 2016.

[162] Y. Li, S. Zhang, and L. Zhang, "Mining location-aware discriminative blocklets for recognizing landmark architectures," Multimedia Systems, vol. 22, no. 4, pp. 455-464, 2016.

[163] B. Liu, Y. Li, S. Zhang, and X. Ye, "Healthy human sitting posture estimation in RGB-D scenes using object context," Multimedia Tools and Applications, vol. 76, no. 8, pp. 10721-10739, 2017.

[164] C. A. Coello Coello, "Theoretical and numerical constrainthandling techniques used with evolutionary algorithms: a survey of the state of the art," Computer Methods in Applied Mechanics and Engineering, vol. 191, no. 11-12, pp. 12451287, 2002.

[165] C. A. Coello Coello and E. Mezura Montes, "Constrainthandling in genetic algorithms through the use of dominance-based tournament selection," Advanced Engineering Informatics, vol. 16, no. 3, pp. 193-203, 2002.

[166] J. S. Arora, "Introduction to design," Introduction to Optimum Design, vol. 55, pp. 1-14, 2004.

[167] A. D. Belegundu and J. S. Arora, "A study of mathematical programming methods for structural optimization. part I: theory," International Journal for Numerical Methods in Engineering, vol. 21, no. 9, pp. 1583-1599, 1985.

[168] Q. He and L. Wang, "An effective co-evolutionary particle swarm optimization for constrained engineering design problems," Engineering Applications of Artificial Intelligence, vol. 20 , no. 1 , pp. 89-99, 2007.

[169] C. A. Coello Coello, "Use of a self-adaptive penalty approach for engineering optimization problems," Computers in Industry, vol. 41, no. 2, pp. 113-127, 2000.

[170] M. Mahdavi, M. Fesanghary, and E. Damangir, "An improved harmony search algorithm for solving optimization problems," Applied Mathematics and Computation, vol. 188, no. 2, pp. 1567-1579, 2007.

[171] A. Kaveh and M. Khayatazad, "A new meta-heuristic method: ray optimization," Computers \& Structures, vol. 112, no. 4, pp. 283-294, 2012.

[172] K. M. Ragsdell and D. T. Phillips, "Optimal design of a class of welded structures using geometric programming," Journal of Engineering for Industry, vol. 98, no. 3, pp. 1021-1025, 1976.

[173] F.-z. Huang, L. Wang, and Q. He, "An effective co-evolutionary differential evolution for constrained optimization," Applied Mathematics and Computation, vol. 186, no. 1, pp. 340-356, 2007. 
[174] G. G. Wang, "Adaptive response surface method using inherited Latin hypercube design points," Journal of $\mathrm{Me}$ chanical Design, vol. 125, no. 2, pp. 210-220, 2003.

[175] A. H. Gandomi, X.-S. Yang, and A. H. Alavi, "Cuckoo search algorithm: a metaheuristic approach to solve structural optimization problems," Engineering with Computers, vol. 29, no. 1, pp. 17-35, 2013.

[176] M.-Y. Cheng and D. Prayogo, "Symbiotic organisms search: a new metaheuristic optimization algorithm," Computers \& Structures, vol. 139, pp. 98-112, 2014. 